TRANSACTIONS OF THE

AMERICAN MATHEMATICAL SOCIETY

Volume 363, Number 11, November 2011, Pages 5605-5648

S 0002-9947(2011)05106-8

Article electronically published on June 15, 2011

\title{
DEFORMATIONS OF FINITE CONFORMAL ENERGY: BOUNDARY BEHAVIOR AND LIMIT THEOREMS
}

\author{
TADEUSZ IWANIEC AND JANI ONNINEN
}

\begin{abstract}
We study homeomorphisms $h: \mathbb{X} \stackrel{\text { onto }}{\longrightarrow} \mathbb{Y}$ between two bounded domains in $\mathbb{R}^{n}$ having finite conformal energy

$$
\mathcal{E}[h]=\int_{\mathbb{X}}\|D h(x)\|^{n} \mathrm{~d} x<\infty, \quad h \in \mathscr{W}^{1, n}(\mathbb{X}, \mathbb{Y}) .
$$

We consider the behavior of such mappings, including continuous extension to the closure of $\mathbb{X}$ and injectivity of $h: \overline{\mathbb{X}} \rightarrow \overline{\mathbb{Y}}$. In general, passing to the weak $\mathscr{W}^{1, n}$-limit of a sequence of homeomorphisms $h_{j}: \mathbb{X} \rightarrow \mathbb{Y}$ one loses injectivity. However, if the mappings in question have uniformly bounded $\mathscr{L}^{1}$-average of the inner distortion, then, for sufficiently regular domains $\mathbb{X}$ and $\mathbb{Y}$, their limit map $h: \mathbb{X} \stackrel{\text { onto }}{\longrightarrow} \mathbb{Y}$ is a homeomorphism. Moreover, the inverse map $f=h^{-1}: \mathbb{Y} \stackrel{\text { onto }}{\longrightarrow} \mathbb{X}$ enjoys finite conformal energy and has integrable inner distortion as well.
\end{abstract}

\section{Contents}

1. Introduction

2. An example

3. Oscillation inequalities

4. A bit of topology of domains in $\mathbb{R}^{n}$

4.1. Gaps in $\mathbb{R}^{n}$

4.2. Unicoherence

4.3. Filling in the gaps

4.4. Disconnection of $\mathbb{R}^{n}$

4.5. Domains of finite connectivity

5. The potential function and its level sets

6. A counterexample to the continuous extension

and the proof of Theorem 1.3

6.1. A counterexample (volcanic hills of finite energy) 5619

6.2. Proof of Theorem $1.3 \quad 5621$

7. Estimates of the distance to the boundary 5623

7.1. The essential diameter 5623

7.2. The modulus of the distance to $\partial \mathbb{Y}_{i}$

Received by the editors August 4, 2008 and, in revised form, May 13, 2009.

2000 Mathematics Subject Classification. Primary 35J15, 35J70.

Key words and phrases. Boundary behavior of homeomorphisms, limit theorems, energy integrals, quasiconformal hyperelasticity.

The first author was supported by NSF grant DMS-0800416.

The second author was supported by NSF grant DMS-0701059.

(C)2011 American Mathematical Society Reverts to public domain 28 years from publication 
7.3. Proof of Theorem 7.1 5624

7.3.1. Estimates near the inner component.

7.3.2. Estimates near the outer component 5627

8. Estimates near the monotone boundary 5

8.1. Monotone domains 5

8.2. An example 5630

8.3. Estimates near a monotone boundary, proof of Theorem 1.2

9. Remarks on the existence of a minimizing map 5635

9.1. Contenders 5635

9.2. The image of $\mathbb{X}$ under the extremal map 5635

9.3. BV-right inverse of the limit, proof of Theorem 1.4

10. Compression-expansion process 5638

References 5645

\section{INTRODUCTION}

The present paper is a sequel of the authors' endeavour to inaugurate a little theory of quasiconformal hyperelasticity [4, 28, 31, 33, 32. The subject matter is about mappings $h: \mathbb{X} \stackrel{\text { onto }}{\longrightarrow} \mathbb{Y}$ between two domains $\mathbb{X}, \mathbb{Y} \subset \mathbb{R}^{n}, n \geqslant 2$. We make three standing assumptions on the domains and maps under consideration:

- $\mathbb{X}$ and $\mathbb{Y}$ will be bounded domains of finite connectivity. Precisely, $\mathbb{R}^{n} \backslash \mathbb{X}=$ $\mathbb{X}_{0} \cup \mathbb{X}_{1} \cup \ldots \cup \mathbb{X}_{\ell}$ and $\mathbb{R}^{n} \backslash \mathbb{Y}=\mathbb{Y}_{0} \cup \mathbb{Y}_{1} \cup \ldots \cup \mathbb{Y}_{\ell}$, where $\mathbb{X}_{0}$ and $\mathbb{Y}_{0}$ are the unbounded components. The outer boundaries of $\mathbb{X}$ and $\mathbb{Y}$ are $\partial \mathbb{X}_{0}$ and $\partial \mathbb{Y}_{0}$, while the other components, $\partial \mathbb{X}_{i}$ and $\partial \mathbb{Y}_{i}, i=1, \ldots, \ell$, will be referred to as inner boundaries.

- The mappings $h: \mathbb{X} \stackrel{\text { onto }}{\longrightarrow} \mathbb{Y}$ will be homeomorphisms preserving the orientation and the order of the boundary components. Since $h$ need not be defined on $\partial \mathbb{X}$, the latter requirement means that

$$
h\left\{\partial \mathbb{X}_{i}\right\}=\partial \mathbb{Y}_{i}, \quad i=0,1, \ldots, \ell,
$$

where $h\left\{\partial \mathbb{X}_{i}\right\}$ stands for the cluster set of $h$ over $\partial \mathbb{X}_{i}$. In particular, we are assuming that $h$ takes outer boundary of $\mathbb{X}$ into outer boundary of $\mathbb{Y}$, whereas the inner boundaries will be conveniently renumerated to meet our notation. We denote by $\mathcal{H}(\mathbb{X}, \mathbb{Y})$ a collection of all such homeomorphisms

$$
\begin{aligned}
\mathcal{H}(\mathbb{X}, \mathbb{Y})= & \{h: \mathbb{X} \stackrel{\text { onto }}{\longrightarrow} \mathbb{Y}: \text { homeomorphisms preserving } \\
& \text { orientation and the boundary components }\} .
\end{aligned}
$$

It may be noted that the inverse map, denoted by $f=h^{-1}: \mathbb{Y} \stackrel{\text { onto }}{\longrightarrow} \mathbb{X}$, lies in $\mathcal{H}(\mathbb{Y}, \mathbb{X})$.

- As regards the regularity, we assume that $h$ belongs to the Sobolev class $\mathscr{W}^{1, n}(\mathbb{X}, \mathbb{Y})$ and has nonnegative Jacobian determinant. The conformal energy of $h=\left(h^{1}, \ldots, h^{n}\right)$ is then defined by

$$
\begin{aligned}
\mathcal{E}[h] & =\int_{\mathbb{X}}\|D h(x)\|^{n} \mathrm{~d} x \\
& \geqslant n^{\frac{n}{2}} \int_{\mathbb{X}} J(x, h) \mathrm{d} x=n^{\frac{n}{2}}|\mathbb{Y}|,
\end{aligned}
$$


where the Jacobian matrix $D h=\left[\frac{\partial h^{i}}{\partial x_{j}}\right] \in \mathbb{R}^{n \times n}$, referred to as the deformation gradient, has nonnegative Jacobian determinant, $J(x, h)=\operatorname{det} D h(x)$ $\geqslant 0$. Hereafter we use the Hilbert Schmidt norm of a matrix, so

$$
\|D h\|^{2}=\sum_{1 \leqslant i, j \leqslant n}\left|\frac{\partial h^{i}}{\partial x_{j}}\right|^{2}=\operatorname{Tr}\left[D^{*} h D h\right] .
$$

Denote by $\mathcal{E}(\mathbb{X}, \mathbb{Y})$ the class of mappings $h \in \mathcal{H}(\mathbb{X}, \mathbb{Y})$ having finite conformal energy

$$
\mathcal{E}(\mathbb{X}, \mathbb{Y})=\left\{h \in \mathcal{H}(\mathbb{X}, \mathbb{Y}): \int_{\mathbb{X}}\|D h\|^{n}<\infty\right\} .
$$

In discussing the inverse map $f=h^{-1}: \mathbb{Y} \stackrel{\text { onto }}{\longrightarrow} \mathbb{X}$ caution must be excercised because it may not have finite conformal energy on $\mathbb{Y}$, unless the inner distortion function of $h$ is integrable; see Proposition 1.1.

In dimension $n=2$ the Dirichlet integral in (3) is central in the theory of harmonic maps [18. Because of conformal invariance we call $\mathscr{E}[h]$ the conformal energy of $h$ or, sometimes, $n$-harmonic energy. In higher dimensions, the $n$-harmonic alternative to the classical Dirichlet integral has drawn the attention of researchers in Geometric Function Theory [55, 7, 25, 62, 56, 29.

1. Boundary Behavior. Let two bounded domains $\mathbb{X}$ and $\mathbb{Y}$ of the same (finite) connectivity be given. We assume that the class $\mathcal{E}(\mathbb{X}, \mathbb{Y})$ is nonempty; see 33 for some results concerning the existence of such mappings.

Theorem 1.1. Suppose $\mathbb{X}$ and $\mathbb{Y}$ have at least two boundary components, $\ell \geqslant 1$. Then there exists a continuous function $\eta=\eta_{\mathbb{X}, \mathbb{Y}}(x)$ defined on $\overline{\mathbb{X}}$, positive on $\mathbb{X}$ and vanishing on $\partial \mathbb{X}$, such that

$$
\operatorname{dist}(h(x), \partial \mathbb{Y}) \leqslant \eta_{\mathbb{X}, \mathbb{Y}}(x)\|D h\|_{\mathscr{L}^{n}(\mathbb{X})} \quad \text { for all } h \in \mathcal{E}(\mathbb{X}, \mathbb{Y}) .
$$

This also holds if $\mathbb{X}$ and $\mathbb{Y}$ have only outer boundary, $\ell=0$, provided $\mathbb{X}$ is incompressible.

Definition 1.1. A domain $\mathbb{X} \subset \mathbb{R}^{n}$ is said to be (locally) compressible (to a point) if for every compact set $\mathfrak{X} \subset \mathbb{X}$ there exist homeomorphisms $g_{j}: \mathbb{X} \stackrel{\text { onto }}{\longrightarrow} \mathbb{X}$ such that $\lim _{j \rightarrow \infty} \operatorname{diam} g_{j}(\mathfrak{X})=0$. Otherwise we say that $\mathbb{X}$ is incompressible.

A ball $\mathbb{B} \subset \mathbb{R}^{n}$ or $\mathbb{B} \backslash \mathbb{R}^{k}, 0 \leqslant k \leqslant n-2$, is locally compressible. Each has a single boundary component. A torus, on the other hand, is incompressible and still has only one boundary component; see Section 10 for a brief discussion on this concept.

Specific bounds for the function $\eta_{\mathbb{X}, \mathbb{Y}}$ are available, provided the target domain $\mathbb{Y}$ has some geometric regularity such as graph domains, polyhydra or more general monotone domains.

Definition 1.2. A bounded domain $\mathbb{Y} \subset \mathbb{R}^{n}$ of finite connectivity is said to be monotone if for each boundary component $\Gamma \subset \partial \mathbb{Y}$ all the level sets

$$
\Gamma^{\epsilon}=\{y \in \mathbb{Y}: \operatorname{dist}(y, \Gamma)=\epsilon\}
$$

are connected for sufficiently small $\epsilon>0$.

We shall see that graph domains are monotone; see Corollary 10.1 . 
Theorem 1.2. Under the assumption of Theorem 1.1, if $\mathbb{Y}$ is monotone, then

$$
\eta_{\mathbb{X}, \mathbb{Y}}(x) \leqslant C_{\mathbb{X}, \mathbb{Y}} \log ^{-1}\left(e+\frac{\operatorname{diam} \mathbb{X}}{\operatorname{dist}(x, \partial \mathbb{X})}\right) .
$$

No specific bounds like this can be obtained without restrictions on $\partial \mathbb{Y}$; see Example 8.1.

As in the theory of qusiconformal mappings [63, 52, 39, in order to extend a given map $h \in \mathcal{E}(\mathbb{X}, \mathbb{Y})$ continuously to the closure of $\mathbb{X}$, we must assume some geometric regularity of $\partial \mathbb{X}$ and $\partial \mathbb{Y}$.

Definition 1.3. The boundary $\partial \mathbb{X}$ is said to be locally quasiconformally flat if every point in $\partial \mathbb{X}$ has a neighborhood $\mathbb{U} \subset \mathbb{R}^{n}$ and a homeomorphism $g: \mathbb{U} \cap \overline{\mathbb{X}} \stackrel{\text { onto }}{\longrightarrow}$ $\left\{x:|x|<1\right.$ and $\left.x_{n} \geqslant 0\right\}$ which is quasiconformal on $\mathbb{U} \cap \mathbb{X} 1$; see 63 .

Definition 1.4. The boundary $\partial \mathbb{Y}$ is a neighborhood retract [11] if there is a neighborhood $\mathbb{V} \subset \mathbb{R}^{n}$ of $\partial \mathbb{Y}$ and a continuous map $\chi: \mathbb{V} \rightarrow \partial \mathbb{Y}$ which is an identity on $\partial \mathbb{Y}$.

Theorem 1.3. Let $\mathbb{X}$ and $\mathbb{Y}$ be bounded domains of finite connectivity. Suppose $\partial \mathbb{X}$ is locally quasiconformally flat and $\partial \mathbb{Y}$ is a neighborhood retract. Then every $h \in \mathcal{E}(\mathbb{X}, \mathbb{Y})$ extends to a continuous map $h: \overline{\mathbb{X}} \stackrel{\text { onto }}{\longrightarrow} \overline{\mathbb{Y}}$.

This theorem fails if $\partial \mathbb{Y}$ is not a neighborhood retract, even when $\mathbb{X}$ is a smooth domain; see Section 6.1.

2. Distortion functions. The central objects in quasiconformal theory are the distortion functions.

Definition 1.5. Let $h: \mathbb{X} \rightarrow \mathbb{R}^{n}$ be a mapping of Sobolev class $\mathscr{W}_{\text {loc }}^{1,1}\left(\mathbb{X}, \mathbb{R}^{n}\right)$ with $J(x, h) \geqslant 0$, not necessarily a homeomorphism or continuous. The outer and inner distortion functions of $h$ are defined by the rules:

$$
\begin{gathered}
K_{O}(x, h)= \begin{cases}\frac{1}{\sqrt{n^{n}}} \frac{\|D h(x)\|^{n}}{J(x, h)} & \text { if } J(x, h)>0, \\
1 & \text { if } J(x, h)=0 \text { and } D h(x)=0, \\
\infty & \text { if } J(x, h)=0 \text { and } D h(x) \neq 0,\end{cases} \\
K_{I}(x, h)= \begin{cases}\frac{1}{\sqrt{n^{n}}} \frac{\left\|D^{\sharp} h(x)\right\|^{n}}{J(x, h)^{n-1}} & \text { if } J(x, h)>0, \\
1 & \text { if } J(x, h)=0 \text { and } D^{\sharp} h(x)=0, \\
\infty & \text { if } J(x, h)=0 \text { and } D^{\sharp} h(x) \neq 0 .\end{cases}
\end{gathered}
$$

Here $D^{\sharp} h(x)$ stands for the matrix of cofactors of $D h(x)$, which is determined by Crammer's rule $D^{\sharp} h(x) D h(x)=J(x, h) I$.

As regards the cases $K_{O}(x, h)=\infty$ or $K_{I}(x, h)=\infty$, these will not occur later on. Indeed, we will be dealing only with mappings of finite distortions. If $K_{O}(x, h)$ (or $\left.K_{I}(x, h)\right)$ is finite almost everywhere, then we say that $h$ has finite outer (inner) distortion, respectively. A map $h$ is said to have integrable inner distortion if $\int_{\mathbb{X}} K_{I}(x, h) \mathrm{d} x<\infty$. Obviously, we have a pointwise inequality

$$
K_{I}(x, h) \leqslant K_{O}^{n-1}(x, h) .
$$

Thus, if $K_{O}(x, h) \in \mathscr{L}^{n-1}(\mathbb{X})$, then $K_{I}(x, h) \in \mathscr{L}^{1}(\mathbb{X})$. This brings us to a result in [27, 53, 17, 33]. 
Proposition 1.1. Let $\mathbb{X}$ and $\mathbb{Y}$ be arbitrary bounded domains in $\mathbb{R}^{n}$ and $h: \mathbb{X} \stackrel{\text { onto }}{\longrightarrow}$ $\mathbb{Y}$ an orientation preserving homeomorphism of Sobolev class $\mathscr{W}_{\operatorname{loc}}^{1, n}(\mathbb{X}, \mathbb{Y})$ with integrable inner distortion. Then the inverse map $f=h^{-1}: \mathbb{Y} \stackrel{\text { onto }}{\longrightarrow} \mathbb{X}$ belongs to $\mathscr{W}^{1, n}(\mathbb{Y}, \mathbb{X})$, it has integrable inner distortion, and we have the identities

$$
\begin{aligned}
& \int_{\mathbb{Y}}\|D f(y)\|^{n} \mathrm{~d} y=n^{\frac{n}{2}} \int_{\mathbb{X}} K_{I}(x, h) \mathrm{d} x, \\
& n^{\frac{n}{2}} \int_{\mathbb{Y}} K_{I}(y, f) \mathrm{d} y=\int_{\mathbb{X}}\|D h(x)\|^{n} \mathrm{~d} x .
\end{aligned}
$$

The Jacobian determinants $J(x, h)$ and $J(y, f)$ are positive almost everywhere. In particular, both $h$ and $f$ have finite outer distortion.

We now introduce a polyconvex variational integral for a homeomorphism $h$ : $\mathbb{X} \stackrel{\text { onto }}{\longrightarrow} \mathbb{Y}$,

$$
\mathcal{E}_{T}[h]=\int_{\mathbb{X}}\left[\|D h(x)\|^{n}+n^{\frac{n}{2}} K_{I}(x, h)\right] \mathrm{d} x,
$$

and a similar one for the inverse map $f=h^{-1}: \mathbb{Y} \stackrel{\text { onto }}{\longrightarrow} \mathbb{X}$,

$$
\mathcal{E}_{T}[f]=\int_{\mathbb{Y}}\left[\|D f(y)\|^{n}+n^{\frac{n}{2}} K_{I}(y, f)\right] \mathrm{d} y .
$$

There are two other ways to express these integrals:

$$
\mathcal{E}_{T}[h]=\mathcal{E}_{T}[f]=\int_{\mathbb{X}}\|D h(x)\|^{n} \mathrm{~d} x+\int_{\mathbb{Y}}\|D f(y)\|^{n} \mathrm{~d} y
$$

or, equivalently,

$$
\mathcal{E}_{T}[h]=\mathcal{E}_{T}[f]=n^{\frac{n}{2}} \int_{\mathbb{Y}} K_{I}(y, f) \mathrm{d} y+n^{\frac{n}{2}} \int_{\mathbb{X}} K_{I}(x, h) \mathrm{d} x .
$$

We recall at this point the Teichmüller theory of planar quasiconformal mappings. It amounts, loosely speaking, to studying homeomorphisms with smallest supremum norm of the distortion; for instance,

$$
\mathcal{K}_{h}=\mathcal{K}_{f}=\left\|K_{I}(x, h)\right\|_{\mathscr{L} \infty(\mathbb{X})}+\left\|K_{I}(y, f)\right\|_{\mathscr{L} \infty(\mathbb{Y})} .
$$

Such a connection with (15) is of sufficient interest to call for closer examination of so-called total conformal energy, especially the existence and uniqueness of injective mappings which minimize the minimizer.

3. Total CONFORMAL EnERGy. However, if one takes the challenge to develop the concept of total energy in higher dimensions, one must include mappings which are not necessarily homemorphisms. The first stage of the extension amounts to defining a so-called total energy for arbitrary Sobolev mappings.

Definition 1.6. Let $h: \mathbb{X} \rightarrow \mathbb{R}^{n}$ be any mapping in the Sobolev space $\mathscr{W}_{\text {loc }}^{1,1}\left(\mathbb{X}, \mathbb{R}^{n}\right)$ with $J(x, h) \geqslant 0$. The total energy of $h$ is defined by

$$
\mathcal{E}_{T}[h]=\int_{\mathbb{X}}\left[\|D h(x)\|^{n}+n^{\frac{n}{2}} K_{I}(x, h)\right] \mathrm{d} x .
$$

A major problem we want to address here is the following:

Conjecture 1.1. A mapping $h: \mathbb{X} \rightarrow \mathbb{R}^{n}$ of finite total conformal energy with $J(x, h) \not \equiv 0$ (nonconstant if $n=2$ ) is open and discrete. Its branch set has dimension not greater than $n-2$. 
An affirmative answer is given to this question in dimension $n=2$ [34; see also [26, 45, 35] for partial results in higher dimensions.

Let us introduce the class of mappings with finite total energy

$$
\mathcal{E}_{T}(\mathbb{X}, \mathbb{Y})=\left\{h \in \mathcal{H}(\mathbb{X}, \mathbb{Y}): \mathcal{E}_{T}[h]<\infty\right\} .
$$

Proposition 1.1 tells us that the inverse map $f=h^{-1}: \mathbb{Y} \stackrel{\text { onto }}{\longrightarrow} \mathbb{X}$ lies in $\mathcal{E}_{T}(\mathbb{Y}, \mathbb{X})$, while Theorem 1.3 yields a Carathéodory type extension theorem for homeomorphisms of finite total energy. We refer the reader to [36, 15, 54, 39, 63, 52, for related topics.

Corollary 1.1. Let $\mathbb{X}$ and $\mathbb{Y}$ be bounded domains with locally quasiconformally flat boundaries. Then every $h \in \mathcal{E}_{T}(\mathbb{X}, \mathbb{Y})$ admits a homeomorphic extension to the boundary, again denoted by $h: \overline{\mathbb{X}} \stackrel{\text { onto }}{\longrightarrow} \overline{\mathbb{Y}}$.

We should point out that locally quasiconformally flat boundaries are locally graphs. On the other hand, the boundary of a graph domain is a neighborhood retract. This fact, interesting on its own, is proven in Section 10

4. MAPPIngs OF SMALlEST ENERGY. Before embarking upon formal results, let us familiarize ourselves with some physical interpretations of the problem, especially those carried out in the theory of nonlinear elasticity [2, [5, 6, 16]. The hyperelasticity is concerned with the energy integrals

$$
\mathcal{E}[h]=\int_{\mathbb{X}} \mathbf{E}(x, h, D h) \mathrm{d} x,
$$

where $\mathbf{E}: \mathbb{X} \times \mathbb{Y} \times \mathbb{R}^{n \times n} \rightarrow \mathbb{R}$ satisfies certain conditions characterizing mechanical properties of the material. We shall confine ourselves to the quasiconformally invariant class of integrals, which yields that $E(x, h, D h) \geqslant\|D h\|^{n}$; see 32 for further discussion. The general task in the mathematical models of elasticity is to find a deformation $h \in \mathcal{H}(\mathbb{X}, \mathbb{Y})$ of smallest energy. These deformations are usually found as weak limits of a minimizing sequence. However, in the limiting process we lose the injectivity of $h$. Let us give a few instances of deformations of the type that may appear. One more-or-less concrete realization of such a situation is obtained by hammering a piece of metal. Suppose one strikes $\mathbb{X}$ repeatedly with a hammer while the metal is hot. Each stroke gives rise to a homeomorphism $h_{k}: \mathbb{X} \rightarrow \mathbb{R}^{n}, k=1,2, \ldots$. When passing to a weak limit in $\mathscr{W}^{1, n}(\mathbb{X}, \mathbb{Y})$, say $\lim h_{k}=h: \mathbb{X} \rightarrow \mathbb{R}^{n}$, we lose injectivity. We refer to this incident as a permanent damage in the material. It is nevertheless true that no holes or cracks will emerge in this process. For example, consider the annuli $\mathbb{X}=\left\{x \in \mathbb{R}^{2}: 1 / 2<|x|<2\right\}$ and $\mathbb{Y}=\left\{y \in \mathbb{R}^{2}: 1<|y|<2\right\}$ in the plane. It turns out that a minimizer $h_{\min }: \mathbb{X} \stackrel{\text { onto }}{\longrightarrow} \mathbb{Y}$ of the conformal energy

$$
\mathcal{E}[h]=\int_{\mathbb{X}}\|D h(x)\|^{2} \mathrm{~d} x
$$

takes the form

$$
h_{\min }(x)=\left\{\begin{array}{lll}
\frac{x}{|x|}, & \frac{1}{2}<|x| \leqslant 1, & \text { hammering part } \\
\frac{1}{2}\left(1+|x|^{-2}\right) x, & 1 \leqslant|x| \leqslant 2, & \text { harmonic part. }
\end{array}\right.
$$

Such a minimizer is unique up to a rotation of annuli; see Figure 1 . 

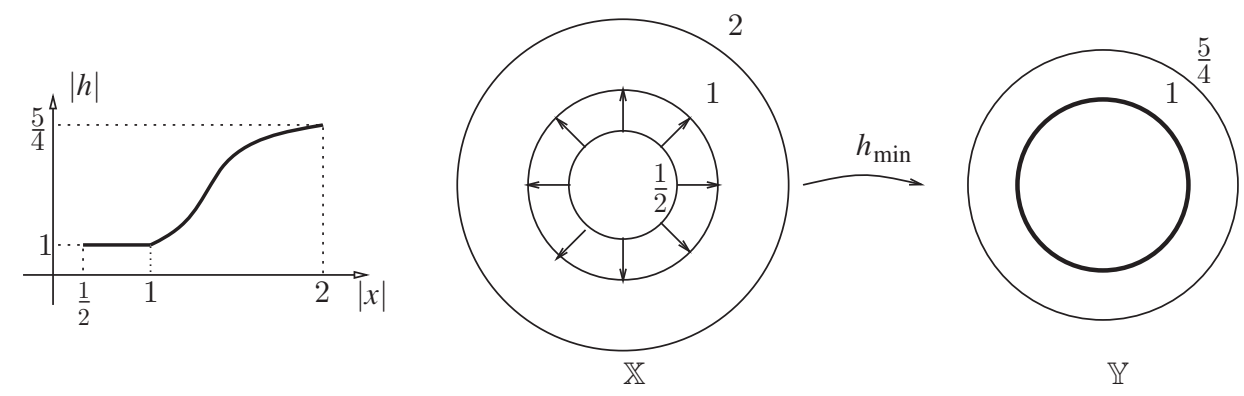

Figure 1. Hammering a portion of the annulus flat.

Note that $h_{\min }$ has a right inverse $f_{\min }(y)=y+y \sqrt{1-|y|^{-2}}$. That is,

$$
h_{\min } \circ f_{\min }=i d: \mathbb{Y} \rightarrow \mathbb{Y} .
$$

A similar type of damage is observed for mappings between annuli in dimensions $n \geqslant 2$, where the energy is the $n$-harmonic integral

$$
\mathcal{E}[h]=\int_{\mathbb{X}}\|D h(x)\|^{n} \mathrm{~d} x .
$$

We refer the reader to 31 for more reading on this subject, with some rather surprising results. The hammering phenomena can only be observed when the annulus $\mathbb{X}$ is conformally too fat, so a part of it must be hammered flat [31. In many ways the above example illustrates the worst behavior that can happen when passing to a weak limit of a minimizing sequence.

Theorem 1.4 (Right inverse of a weak limit). Let $\mathbb{X}$ and $\mathbb{Y}$ be bounded domains of finite connectivity having at least two boundary components. Suppose we are given a sequence $h_{j} \in \mathcal{E}(\mathbb{X}, \mathbb{Y})$ converging weakly in $\mathscr{W}^{1, n}(\mathbb{X}, \mathbb{Y})$ to a map $h \in \mathscr{W}^{1, n}\left(\mathbb{X}, \mathbb{R}^{n}\right)$. Then $h$ is continuous and $\mathbb{Y} \subset h(\mathbb{X}) \subset \overline{\mathbb{Y}}$. Furthermore, there exists a measurable mapping $f: \mathbb{Y} \rightarrow \mathbb{X}$, such that

$$
h \circ f=\mathrm{id}: \mathbb{Y} \rightarrow \mathbb{Y},
$$

everywhere on $\mathbb{Y}$. This right inverse mapping has bounded variation. All the above still hold if $\partial \mathbb{X}$ has only an outer boundary, provided $\mathbb{X}$ is incompressible.

Finally, applying this theorem to mappings $h_{j} \in \mathcal{E}_{T}(\mathbb{X}, \mathbb{Y}) \subset \mathcal{E}(\mathbb{X}, \mathbb{Y})$ and their inverses $f_{j} \in \mathcal{E}_{T}(\mathbb{Y}, \mathbb{X}) \subset \mathcal{E}(\mathbb{Y}, \mathbb{X})$, we conclude with the following.

Corollary 1.2 (Weak compactness). Suppose that bounded domains $\mathbb{X}$ and $\mathbb{Y}$ of finite connectivity have at least two boundary components or are incompressible when having only one boundary component. Then the family

$$
\mathcal{F}_{M}(\mathbb{X}, \mathbb{Y})=\left\{h \in \mathcal{E}_{T}(\mathbb{X}, \mathbb{Y}): \mathcal{E}_{T}[h] \leqslant M\right\}
$$

is compact in weak topology of $\mathscr{W}^{1, n}\left(\mathbb{X}, \mathbb{R}^{n}\right)$. 


\section{AN EXAMPLE}

The following example is well suited for several purposes of the present work.

Example 2.1 (Conformal automorphisms of the unit ball). To every point $a \in$ $\mathbb{B} \subset \mathbb{R}^{n}, a \neq 0$, there corresponds a conformal deformation $h_{a}: \mathbb{B} \stackrel{\text { onto }}{\longrightarrow} \mathbb{B}$, defined by

$$
h_{a}(x)=\tilde{a}+\frac{\left(|\tilde{a}|^{2}-1\right)(x-\tilde{a})}{|x-\tilde{a}|^{2}}, \quad \text { where } \tilde{a}=\frac{a}{|a|^{2}} .
$$

Elementary computation shows that $h(0)=a, h(a)=0$, and

$$
1-|h(x)|^{2}=\left(1-|x|^{2}\right) \frac{|\tilde{a}|^{2}-1}{|x-\tilde{a}|^{2}} .
$$

These mappings and their inverses share the same conformal energy. Indeed, because of conformality, we have

$$
\int_{\mathbb{B}}\left\|D h_{a}(x)\right\|^{n} \mathrm{~d} x=n^{\frac{n}{2}} \int_{\mathbb{B}} J\left(x, h_{a}\right) \mathrm{d} x=n^{\frac{n}{2}}|\mathbb{B}| .
$$

As $a$ approaches one boundary point $a_{\circ} \in \partial \mathbb{B}$, we find that $\left\{h_{a}\right\}$ converges $c$ uniformly (uniformly on compact subsets) to the constant mapping $h(x) \equiv a_{\circ}$, so the injectivity is lost. The loss of injectivity would not have happened if the mappings $h$ were fixed at a given point, say $h(p)=q$ for some $p \in \mathbb{X}$ and $q \in \mathbb{Y}$. This normalization amounts to saying that the mappings take the punctured ball $\mathbb{B} \backslash\{p\}$ onto the punctured ball $\mathbb{B} \backslash\{q\}$. These domains have two boundary components. For more details we refer the reader to Corollary 1.2 and Section 9.3 .

The reader will also notice that $\mathbb{B} \backslash \mathbb{R}^{n-2}, n \geqslant 3$, is still a domain with one boundary component. Moreover, if $a \in \mathbb{R}^{n-2}$, then $h_{a}: \mathbb{B} \backslash \mathbb{R}^{n-2} \stackrel{\text { onto }}{\longrightarrow} \mathbb{B} \backslash \mathbb{R}^{n-2}$. We still lose injectivity in passing to the limit as $|a| \rightarrow 1$. Actually, the reason for losing injectivity of the limit map is that $\mathbb{B} \backslash \mathbb{R}^{n-2}$ is locally compressible to a point; see Definition 1.1. Again, this would not have happened if we drilled a tiny hole through the ball instead of removing a flat hyperplane of codimension 2. A topological torus, so obtained, becomes an incompressible domain with one boundary component.

\section{Oscillation INEQUALities}

Definition 3.1. Let $\Omega$ be a domain in $\mathbb{R}^{n}$. A continuous function $u: \Omega \rightarrow \mathbb{R}$ is said to be monotone 42 if for every compact $\mathbb{F} \subset \Omega$, we have

$$
\min _{\partial \mathbb{F}} u=\min _{\mathbb{F}} u \leqslant \max _{\mathbb{F}} u=\max _{\partial \mathbb{F}} u .
$$

In other words, $u$ satisfies both the maximum and minimum principles 1

Proposition 3.1 (Oscillation on balls). Given concentric balls $\mathbb{B} \subset \lambda \mathbb{B} \subset \mathbb{R}^{n}$, $\lambda>1$, and a continuous monotone function $u \in \mathscr{W}^{1, n}(\lambda \mathbb{B})$, then

$$
[\underset{\mathbb{B}}{\operatorname{osc} u}]^{n} \leqslant \frac{C_{n}}{\log \lambda} \int_{\lambda \mathbb{B}}|\nabla u(x)|^{n} \mathrm{~d} x
$$

\footnotetext{
${ }^{1}$ For Sobolev functions the more relevant concept is that of weak monotonicity [44]. But we do not enter into such generalities, as the need will not arise; see 21], 28], [59, 43].
} 
We include the derivation of this well-known inequality to keep our exposition self-contained.

Proof. We consider a ball $\mathbb{B}$ with radius $r$ and center at the origin, with the usual notation $\mathbb{B}=B_{r}=\{x:|x|<r\}$. In view of monotonicity of $u$ we can write

$$
\underset{\mathbb{B}}{\operatorname{osc}} u \leqslant \underset{B_{\rho}}{\operatorname{osc}} u=\underset{\partial B_{\rho}}{\operatorname{osc}} u \quad \text { for } r \leqslant \rho \leqslant \lambda r .
$$

Next we apply Sobolev's imbedding inequality on spheres,

$$
[\underset{\mathbb{B}}{\operatorname{osc} u}]^{n} \preccurlyeq \rho \int_{\partial B_{\rho}}|\nabla u|^{n} \quad \text { for all } r \leqslant \rho \leqslant \lambda r \mathbb{2}
$$

We divide by $\rho$ and integrate from $r$ to $\lambda r$,

$$
[\underset{\mathbb{B}}{\operatorname{osc} u}]^{n} \int_{r}^{\lambda r} \frac{\mathrm{d} \rho}{\rho} \preccurlyeq \int_{r}^{\lambda r}\left(\int_{\partial B_{\rho}}|\nabla u|^{n}\right) \mathrm{d} \rho \leqslant \int_{\lambda \mathbb{B}}|\nabla u|^{n} .
$$

Hence the inequality (24) follows.

With a little additional work Proposition 3.1 gives a uniform continuity estimate of $u$.

Lemma 3.1 (Modulus of continuity). Let $u \in \mathscr{W}^{1, n}(\Omega)$ be continuous and monotone. Then for every concentric ball $\mathbb{B} \subset 2 \mathbb{B} \subset \Omega$ we have

$$
\left|u\left(x_{1}\right)-u\left(x_{2}\right)\right|^{n} \leqslant \frac{C_{n} \int_{2 \mathbb{B}}|\nabla u(x)|^{n} \mathrm{~d} x}{\log \left(e+\frac{\operatorname{diam} \mathbb{B}}{\left|x_{1}-x_{2}\right|}\right)}, \quad \text { for } \quad x_{1}, x_{2} \in \mathbb{B} .
$$

Remark 3.1. Monotone functions in the Sobolev class $\mathscr{W}_{\text {loc }}^{1, p}(\Omega)$ are differentiable almost everywhere if $p>n-1, n \geqslant 3$, but not necessary if $p=n-1$. This is also true for $p \geqslant 1$ if $n=2$; see [47, [19], 22], 44] and [59].

Another useful inference from Proposition 3.1 is the oscillation of monotone functions around a puncture; that is, in a domain $\Omega$ with one point $x_{\circ} \in \Omega$ removed.

Lemma 3.2 (Oscillation on spheres). Suppose a continuous function $u: \Omega \backslash\left\{x_{0}\right\} \rightarrow$ $\mathbb{R}$ belongs to $\mathscr{W}^{1, n}\left(\Omega \backslash\left\{x_{0}\right\}\right)$ and is monotone. Then for every concentric ball $\mathbb{B}=B\left(x_{0}, r\right) \subset B\left(x_{0}, 3 r\right)=3 \mathbb{B} \subset \Omega$ we have

$$
\left[\begin{array}{c}
\operatorname{osc} u \\
\partial \mathbb{B}
\end{array}\right]^{n} \preccurlyeq \int_{3 \mathbb{B}}|\nabla u(x)|^{n} \mathrm{~d} x
$$

where the implied constant depends only on $n$.

Proof. It is geometrically clear that every two points $x_{1}, x_{2} \in \partial \mathbb{B}$ can be connected by two overlapping closed balls $\overline{B_{1}}$ and $\overline{B_{2}}$ of the same radius as $\mathbb{B}$. Precisely, $x_{1}, x_{2} \in \overline{B_{1}} \cup \overline{B_{2}}, \overline{B_{1}} \cap \overline{B_{2}} \neq \emptyset$ and $\sqrt{2} B_{1} \cup \sqrt{2} B_{2} \subset 3 B \backslash\left\{x_{\circ}\right\}$. Hence

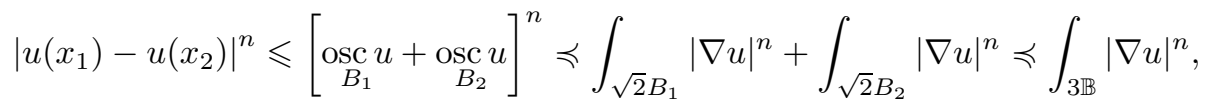

as desired.

\footnotetext{
${ }^{2}$ The symbol $\mathbf{A} \preccurlyeq \mathbf{B}$ is merely an abbreviation for the inequality $\mathbf{A} \leqslant c \cdot \mathbf{B}$ in which $c>0$, called the implied constant, plays no role. The quantities of interest to us are $\mathbf{A} \geqslant 0$ and $\mathbf{B} \geqslant 0$. The implied constant changes from line to line and can be easily identified from the context. Here $c$ depends on the dimension only.
} 
Remark 3.2. The reader may wish to infer from this lemma that $u$ has a limit at the point $x_{\circ}$, possibly $+\infty$ or $-\infty$. In this way $u$ becomes a continuous function on $\Omega$ with values in the extended real line $\widehat{\mathbb{R}}=\mathbb{R} \cup\{-\infty,+\infty\}$. The following example $u(x)=\log \log |x|$ illustrates that $u$ need not be bounded.

It is worth further pursuing Lemma 3.2 on the assumption that $u: \Omega \backslash\left\{x_{0}\right\} \rightarrow \mathbb{R}^{n}$ is a continuous injection. One can easily see, via additional topological arguments, that $u$ has a finite limit at the puncture. Precisely, we have

Theorem 3.1 (Removability of punctures). Let $\mathbb{X}$ be a domain in $\mathbb{R}^{n}, \mathbb{X}^{\prime}=\mathbb{X} \backslash\left\{x_{\circ}\right\}$ with $x_{\circ} \in \mathbb{X}$, and let $h: \mathbb{X}^{\prime} \stackrel{\text { onto }}{\longrightarrow} \mathbb{Y}^{\prime} \subset \mathbb{R}^{n}$ be a homeomorphism of Sobolev class $\mathscr{W}^{1, n}\left(\mathbb{X}^{\prime}, \mathbb{R}^{n}\right)$. Then $h$ extends to a homeomorphism $h: \mathbb{X} \stackrel{\text { onto }}{\longrightarrow} \mathbb{Y}$ in the Sobolev space $\mathscr{W}^{1, n}\left(\mathbb{X}, \mathbb{R}^{n}\right)$, where $\mathbb{Y}=\mathbb{Y}^{\prime} \cup\left\{y_{\circ}\right\}$ and $y_{\circ}=h\left(x_{\circ}\right)$.

This should be compared with Proposition 5.1 in 33 in which the singleton $\left\{x_{0}\right\}$ is replaced by a closed set of zero dimension.

Lemma 3.3 (Vanishing oscillations). Let $\mathbb{B}$ be the unit ball in $\mathbb{R}^{n}$ and let $h \in$ $\mathscr{W}_{\text {loc }}^{1, n}\left(\mathbb{B}, \mathbb{R}^{n}\right)$. Then there is a decreasing sequence of radii $r_{\nu} \rightarrow 0$ such that

1. $h$ restricted to $\mathbb{S}_{r_{\nu}}=\left\{x ;|x|=r_{\nu}\right\}$ is continuous,

2. $\operatorname{diam} h\left(\mathbb{S}_{r_{\nu}}\right) \rightarrow 0$ as $r_{\nu} \rightarrow 0$.

Proof. Upon a suitable choice of a representative of $h \in \mathscr{W}_{\text {loc }}^{1, n}\left(\mathbb{B}, \mathbb{R}^{n}\right)$, we may apply Sobolev's imbedding theorem on spheres to conclude that for almost every sphere $\mathbb{S}_{r}, 0<r<1$, the map $h: \mathbb{S}_{r} \rightarrow \mathbb{R}^{n}$ is continuous. Moreover, by Fubini's theorem we find that

$$
\int_{0}^{r}\left[\operatorname{diam} h\left(\mathbb{S}_{t}\right)\right]^{n} \frac{\mathrm{d} t}{t} \leqslant C_{n} \int_{|x|<r}|D h(x)|^{n} \mathrm{~d} x .
$$

Hence we infer that $\liminf _{t \rightarrow 0}\left[\operatorname{diam} h\left(\mathbb{S}_{t}\right)\right]=0$, which yields assertion 2 in Lemma 3.3.

\section{A bit of topology of domains in $\mathbb{R}^{n}$}

In spite of vast literature, predominantly analytical in nature, the topological aspects of domains in $\mathbb{R}^{n}$ seem not to have been treated with sufficient clarity and rigor. The goal of this section is to provide an account for the sometimes cumbersome details associated with domains in $\mathbb{R}^{n}$. The results here incorporate all our intuition concerning connectedness and boundary components. The best general reference is the monograph by K. Kuratowski, [37, 38.

4.1. Gaps in $\mathbb{R}^{n}$. A domain $\Omega \subset \mathbb{R}^{n}$ is any open connected subset of $\mathbb{R}^{n}$. The complement of $\Omega$ will be denoted by $\mathbb{G}=\mathbb{R}^{n} \backslash \Omega$, and the common boundary by

$$
\Gamma=\partial \Omega=\partial \mathbb{G} .
$$

We consider the collection $\left\{\mathbb{G}_{i}\right\}_{i \in I}$ of all components of $\mathbb{G}=\mathbb{R}^{n} \backslash \Omega$. The index set $I$ may be uncountable. By definition, each $\mathbb{G}_{i}$ is a maximal connected subset of $\mathbb{G}$, properly contained in no other connected subset of $\mathbb{G}$. Characteristically for a domain, the sets $\mathbb{G}_{i}$ are mutually disjoint closed sets whose union is also closed:

$$
\mathbb{G}=\bigcup_{1 \in I} \mathbb{G}_{i}=\mathbb{R}^{n} \backslash \Omega \text {. }
$$

We shall view those sets as gaps in $\mathbb{R}^{n}$. It is worthwhile to begin with the following decomposition of the boundary of $\mathbb{G}$. 
Lemma 4.1. We have

$$
\partial \mathbb{G}=\bigcup_{i \in I} \partial \mathbb{G}_{i} .
$$

4.2. Unicoherence. For further relations between $\Omega$ and the components $\mathbb{G}_{i} \subset$ $\mathbb{G}$ we will have to appeal to the unicoherence property of $\mathbb{R}^{n}$, which is one of the most effective tools when dealing with questions of connectedness. We state it as follows.

Theorem 4.1 (Unicoherence of $\mathbb{R}^{n}$ ). Let $\mathbb{A}$ and $\mathbb{B}$ be domains in $\mathbb{R}^{n}$ such that $\mathbb{A} \cup \mathbb{B}=\mathbb{R}^{n}$. Then $\mathbb{A} \cap \mathbb{B}$ is connected.

Proof. We employ the Mayer-Vietoris long exact sequence:

$$
\mathscr{H}_{1}(\mathbb{A} \cup \mathbb{B}) \longrightarrow \mathscr{H}_{0}(\mathbb{A} \cap \mathbb{B}) \longrightarrow \mathscr{H}_{0}(\mathbb{A}) \oplus \mathscr{H}_{0}(\mathbb{B}) \longrightarrow \mathscr{H}_{0}(\mathbb{A} \cup \mathbb{B}) \longrightarrow 0 .
$$

Since $\mathbb{A}, \mathbb{B}$ and $\mathbb{A} \cup \mathbb{B}=\mathbb{R}^{n}$ are path connected, their homology in the zero dimension is $\mathbf{Z}=\{0, \pm 1, \pm 2, \ldots\}$. Also, we have $\mathscr{H}_{1}(\mathbb{A} \cup \mathbb{B})=\mathscr{H}_{1}\left(\mathbb{R}^{n}\right)=0$. This makes our sequence

$$
0 \longrightarrow \mathscr{H}_{0}(\mathbb{A} \cap \mathbb{B}) \stackrel{\text { injection }}{\longrightarrow} \mathbf{Z} \oplus \mathbf{Z} \stackrel{\text { onto }}{\longrightarrow} \mathbf{Z} \longrightarrow 0 .
$$

By the exactness of this sequence we infer that $\mathscr{H}_{0}(\mathbb{A} \cap \mathbb{B}) \approx \mathbf{Z}$, which means that $\mathbb{A} \cap \mathbb{B}$ is connected.

Here is a direct generalization of the unicoherence theorem.

Corollary 4.1. Let $\mathbb{A}$ and $\mathbb{B}$ be connected subsets of $\mathbb{R}^{n}$ such that $\mathbb{A} \cup \mathbb{B}=\mathbb{R}^{n}$. Then $\overline{\mathbb{A}} \cap \overline{\mathbb{B}}$ is connected.

Corollary 4.2. A set $\mathbb{A}$ and its complement $\mathbb{B}=\mathbb{R}^{n} \backslash \mathbb{A}$ have a common boundary, $\partial \mathbb{A}=\partial \mathbb{B}=\overline{\mathbb{A}} \cap \overline{\mathbb{B}}$. If, in addition, $\mathbb{A}$ and $\mathbb{B}$ are connected, then so is the common boundary.

As a simple consequence of unicoherence of $\mathbb{R}^{n}$ and De Morgan's law, we obtain

Lemma 4.2. The intersection of a finite family of domains whose complements are mutually disjoint is a domain.

Hint. It suffices to consider a family of two such domains. The general case will result by induction, with the details being left to the reader.

4.3. Filling in the gaps. A somewhat dual statement to Lemma 4.2 asserts that filling in the gaps does not disconnect the region. Precisely, this means that:

Lemma 4.3. For every subcollection $\left\{\mathbb{G}_{\lambda}\right\}_{\lambda \in \Lambda}, \Lambda \subset J$, the set

$$
\Omega \cup \bigcup_{\lambda \in \Lambda} \mathbb{G}_{\lambda}=\mathbb{R}^{n} \backslash \bigcup_{\lambda \notin \Lambda} \mathbb{G}_{\lambda} \quad \text { is connected (not necessarily open). }
$$

This set is also open if the index set I is finite.

Proof. We express this set as

$$
\Omega \cup \bigcup_{\lambda \in \Lambda} \mathbb{G}_{\lambda}=\mathbb{F} \cup \bigcup_{\lambda \in \Lambda} \mathbb{G}_{\lambda}, \quad \text { where } \mathbb{F} \stackrel{\text { def }}{=} \Omega \cup \bigcup_{\lambda \in \Lambda} \partial \mathbb{G}_{\lambda} .
$$

By Lemma 4.1 we see that $\Omega \subset \mathbb{F} \subset \Omega \cup \partial \Omega=\bar{\Omega}$, and hence $\mathbb{F}$ is connected. Now each of the components $\mathbb{G}_{\lambda}, \lambda \in \Lambda$, intersects $\mathbb{F}$. Therefore, the union $\mathbb{F} \cup \bigcup_{\lambda \in \Lambda} \mathbb{G}_{\lambda}$ remains connected. As a word of caution the sets $\Omega \cup \bigcup_{\lambda \in \Lambda} \mathbb{G}_{\lambda}$ need not be open, unless the family of unfilled gaps is finite. 
Consider a component $\mathbb{G}_{i} \subset \mathbb{G}$; that is, a gap in $\mathbb{R}^{n}$.

Lemma 4.4. The boundary of each $\mathbb{G}_{i}$ is connected and coincides with a component of $\Gamma=\partial \Omega=\partial \mathbb{G}$.

Proof. We have a decomposition of $\mathbb{R}^{n}$,

$$
\mathbb{R}^{n}=\mathbb{G}_{i} \cup\left(\Omega \cup \bigcup_{\lambda \neq i} \mathbb{G}_{\lambda}\right),
$$

where the set in parentheses is connected, by Lemma 4.3. Now, Corollary 4.2 tells us that the boundary of $\mathbb{G}_{i}$ is connected. It is a subset of $\partial \mathbb{G}$, by Lemma 4.1. Thus $\partial \mathbb{G}_{i}$ lies in one and only one component of $\partial \mathbb{G}$. On the other hand any component of $\partial \mathbb{G}$ which meets $\partial \mathbb{G}_{i}$ must lie in $\partial \mathbb{G}_{i}$. Indeed, suppose $\partial \mathbb{G}_{i} \subset \Gamma_{\circ}$, where $\Gamma_{\circ}$ is a component of $\Gamma=\partial \mathbb{G}$. Since $\mathbb{G}_{i} \cap \Gamma_{\circ} \neq \emptyset$, the union $\mathbb{G}_{i} \cup \Gamma_{\circ}$ is a connected subset of $\mathbb{G}$. This yields $\mathbb{G}_{i} \cup \Gamma_{\circ}=\mathbb{G}_{i}$ and, hence, $\Gamma_{\circ} \subset \mathbb{G}_{i}$. Finally,

$$
\Gamma_{\circ} \subset \mathbb{G}_{i} \cap \partial \mathbb{G} \subset \mathbb{G}_{i} \backslash \operatorname{Int} \mathbb{G} \subset \mathbb{G}_{i} \backslash \operatorname{Int} \mathbb{G}_{i}=\partial \mathbb{G}_{i},
$$

as desired.

Now, knowing that the sets $\partial \mathbb{G}_{i}$ are connected, it follows from Lemma 4.1 that every component of $\Gamma=\partial \Omega$ coincides with the boundary of exactly one gap. Let the components of $\partial \Omega$ be denoted by

$$
\Gamma_{i} \stackrel{\text { def }}{=} \partial \mathbb{G}_{i}, \quad i \in I, \quad \text { the components of } \Gamma=\partial \Omega .
$$

The preceding discussion can be summarized in the following theorem.

Theorem 4.2 (Boundary components). Let $\Omega$ be any domain in $\mathbb{R}^{n}$, so its boundary consists of mutually disjoint closed connected sets $\Gamma_{i}, i \in I$. To every $\Gamma_{i}$ there corresponds one and only one component $\mathbb{G}_{i}$ of $\mathbb{G}=\mathbb{R}^{n} \backslash \Omega$ in such a way that $\partial \mathbb{G}_{i}=\Gamma_{i}$.

4.4. Disconnection of $\mathbb{R}^{n}$. A continuum $\mathbb{K} \subset \mathbb{R}^{n}$ is said to disconnect $\mathbb{R}^{n}$ if $\mathbb{R}^{n} \backslash \mathbb{K}$ is not connected. More generally, let $\Omega$ be any domain in $\mathbb{R}^{n}$. A set $\mathbb{K} \subset \mathbb{R}^{n}$, not necessarily closed, is said to disconnect $\Omega$ if $\Omega \backslash \mathbb{K}$ is not connected.

Theorem 4.3. No set $\mathbb{K} \subset \mathbb{R}^{n}$ of a topological dimension $\operatorname{dim} \mathbb{K} \leqslant n-2$ disconnects any domain. In particular, closed sets in $\mathbb{R}^{n}$ of Hausdorff dimension less than $n-1$ do not disconnect domains.

The classical Jordan Curve Theorem which is generalized to higher dimensions by Brouwer [12], asserts that

Theorem 4.4 (Jordan-Brouwer Separation Theorem). A subset $\mathcal{S} \subset \mathbb{R}^{n}$ which is homeomorphic to $\mathbb{S}^{n-1}$ disconnects $\mathbb{R}^{n}$ into two components. That is, $\mathbb{R}^{n} \backslash \mathcal{S}$ consists of two disjoint connected open sets $\mathbb{U}$ and $\mathbb{V}$ whose common boundary is $\mathcal{S}$.

As a matter of fact, the set $\mathcal{S}$ in Theorem 4.4 can be replaced by any $(n-1)$ dimensional topological manifold, that is, connected and locally homeomorphic to $\mathbb{R}^{n-1}$; see [61, p. 294].

An intrinsic characterization of disconnecting compact sets in $\mathbb{R}^{n}$ is due to K. Borsuk; see [8, 9, 10]. But we shall not enter into this theory here, as the need will not arise. However, the following conclusions will be useful. 
Theorem 4.5. If a compact set $\mathfrak{X} \subset \mathbb{R}^{n}$ disconnects $\mathbb{R}^{n}$, then so does its homeomorphic image in $\mathbb{R}^{n}$. The number of components in $\mathbb{R}^{n} \backslash \mathfrak{X}$ is a topological invariance.

Remark 4.1. Let us also note an obvious fact that a connected open set $\Omega \subset \mathbb{R}^{n} \backslash \mathfrak{X}$ is a component of $\mathbb{R}^{n} \backslash \mathfrak{X}$ if and only if $\partial \Omega \subset \mathfrak{X}$.

Quite recently Theorem 4.4 has been generalized to noninjective images of $\mathbb{S}^{n-1}$; see J. F. Lafont [40, Theorem 1.3].

Theorem 4.6. Let $\mathcal{S} \subset \mathbb{R}^{n}$ be homeomorphic to $\mathbb{S}^{n-1}$ and let $\mathfrak{X} \varsubsetneqq \mathcal{S}$ be a proper compact subset of $\mathcal{S}$. Suppose we are given a continuous map $\Phi: \mathcal{S} \rightarrow \mathbb{R}^{n}$ which is injective on $\mathcal{S} \backslash \mathfrak{X}$ and $\Phi(\mathcal{S} \backslash \mathfrak{X}) \cap \Phi(\mathfrak{X})=\emptyset$. Then:

- The set $\Phi(\mathcal{S})$ disconnects the space $\mathbb{R}^{n}$.

- To every point $p \in \Phi(\mathcal{S} \backslash \mathfrak{X})$ there correspond precisely two connected components of $\subset \mathbb{R}^{n} \backslash \Phi(\mathcal{S})$ which contain $p$ in their closure.

See also [41] for noninjective images of $(n-1)$-manifolds or even more general spaces.

4.5. Domains of finite connectivity. From now on we assume that $\Omega$ is bounded and its boundary $\Gamma=\partial \Omega$ consists of a finite number of mutually disjoint continua, say

$$
\begin{cases}\Gamma=\partial \Omega=\Gamma_{0} \cup \Gamma_{1} \cup \cdots \cup \Gamma_{\ell}, & \ell \geqslant 1, \\ \mathbb{R}^{n} \backslash \Omega=\mathbb{G}_{0} \cup \mathbb{G}_{1} \cup \cdots \cup \mathbb{G}_{\ell}, & \ell \geqslant 1,\end{cases}
$$

where $\Gamma_{i}=\partial \mathbb{G}_{i}, i=0,1, \ldots, \ell$. Hereafter the zero subscript will always stand for the unbounded component of $\mathbb{R}^{n} \backslash \Omega$. We call $\Gamma_{0}=\partial \mathbb{G}_{0}$ the outer boundary of $\Omega$. Note that the corresponding gaps are a positive distance apart:

$$
\min _{0 \leqslant i<j \leqslant \ell} \operatorname{dist}\left(\Gamma_{i}, \Gamma_{j}\right)=\min _{0 \leqslant i<j \leqslant \ell} \operatorname{dist}\left(\mathbb{G}_{i}, \mathbb{G}_{j}\right)>0 .
$$

Such $\Omega$ will henceforth be referred to as an $(\ell+1)$-connected domain. Note that $(\ell+1)$-connectivity is topological invariance; that is, the image $h(\Omega)$ of an $(\ell+1)$ connected domain $\Omega \subset \mathbb{R}^{n}$ under a homeomorphism $h: \Omega \rightarrow \mathbb{R}^{n}$ is an $(\ell+1)$ connected domain. In the case $\ell=0$ the complement of $\Omega$ is connected. Let a positive number $\epsilon$ be chosen and fixed small enough to satisfy

$$
2 \epsilon<\operatorname{dist}\left(\Gamma_{i}, \Gamma_{j}\right), \text { for all } 0 \leqslant i<j \leqslant \ell .
$$

The $\epsilon$ neighborhoods of the gaps, defined by

$$
\mathbb{G}_{i}^{\epsilon}=\left\{x \in \mathbb{R}^{n} ; \operatorname{dist}\left(x, \mathbb{G}_{i}\right)<\epsilon\right\}, \quad i=0,1, \ldots, \ell,
$$

form a disjoint family of domains in $\mathbb{R}^{n}$. Each of them contains exactly one boundary component of $\Omega$, namely $\Gamma_{i} \subset \mathbb{G}_{i}^{\epsilon}$. The boundary of $\mathbb{G}_{i}^{\epsilon}$ (not necessarily connected) is the level set of the distance function to $\mathbb{G}_{i}$. In analogy with the notation $\Gamma_{i}=\partial \mathbb{G}_{i}$, we introduce

$$
\Gamma_{i}^{\epsilon}=\partial \mathbb{G}_{i}^{\epsilon}=\left\{x \in \Omega ; \quad \operatorname{dist}\left(x, \mathbb{G}_{i}\right)=\epsilon\right\} .
$$

In general, $\Gamma_{i}^{\epsilon}$ need not be connected.

Obviously, the boundary of the closure $\overline{\mathbb{G}_{i}^{\epsilon}}=\left\{x \in \mathbb{R}^{n} ; \quad \operatorname{dist}\left(x, \mathbb{G}_{i}\right) \leqslant \epsilon\right\}$ is contained in $\Gamma_{i}^{\epsilon}$. But it may not coincide with $\Gamma_{i}^{\epsilon}$, even for arbitrarily small $\epsilon$; see Figure 2. 


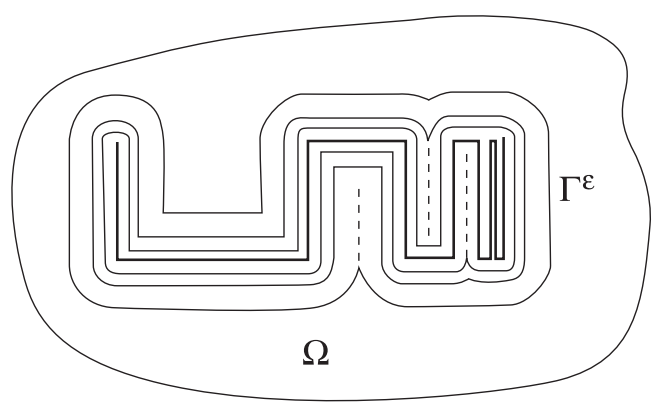

FIGURE 2. Although $\Omega$ is monotone near the snake shaped boundary, the points in the dotted lines have no escape to a farther distance from the snake.

\section{The Potential FunCtion AND ITS LEVEL SETS}

Let $\Omega$ be a bounded domain in $\mathbb{R}^{n}$ whose complement $\Omega_{0}=\mathbb{R}^{n} \backslash \Omega$ is also connected. Obviously, by virtue of unicoherence of $\mathbb{R}^{n}$, the common boundary $\partial \Omega=\partial \Omega_{0}=\bar{\Omega} \cap \Omega_{0}$ is also connected. Let $\Upsilon$ be a continuum in $\Omega$. We consider functions $u \in \mathscr{W}_{0}^{1, p}\left(\mathbb{R}^{n}\right)$ with some fixed exponent $p>n$, such that

$$
u \equiv 1 \quad \text { on } \Upsilon \text { and } u \equiv 0 \text { on } \Omega_{0} .
$$

Among all such functions there is exactly one, denoted by $U$, which minimizes the variational integral

$$
\min \int_{\Omega}\left(1+|\nabla u(y)|^{2}\right)^{\frac{p}{2}} \mathrm{~d} y=\int_{\Omega}\left(1+|\nabla U|^{2}\right)^{\frac{p}{2}} .
$$

This minimizer, referred to as the potential function of the condenser $(\Upsilon, \Omega)$, is $\mathscr{C}^{\infty}$-smooth in $\Omega \backslash \Upsilon$. It enjoys especially pleasing geometric behavior. First, $U$ is continuous in $\mathbb{R}^{n}$, because $p>n$. It follows from the general elliptic theory of variational integrals such as this that

$$
U \in \mathscr{C}^{\infty}(\Omega \backslash \Upsilon) \text { and } 0<U<1 \text { on } \Omega \backslash \Upsilon .
$$

These latter inequalities are a straightforward consequence of strong maximum and minimum principles 25]. Further results demand a few geometric observations concerning the level sets $\Gamma_{c}=\left\{y \in \mathbb{R}^{n} ; \quad U(y)=c\right\}$ for $0 \leqslant c \leqslant 1$. Thus $\Gamma_{0}=\Omega_{0}$ and $\Gamma_{1}=\Upsilon$. The celebrated theorem of Sard [57] comes in handy. Recall that $0<c<1$ is a regular value of $U$ if $\nabla U \neq 0$ on $\Gamma_{c}$. Almost every $c \in(0,1)$ is a regular value. Thus, by the Implicit Function Theorem the corresponding regular level set $\Gamma_{c}$ consists of a finite number of $\mathscr{C}^{\infty}$-smooth closed $(n-1)$-manifolds. In fact $\Gamma_{c}$ is connected, thus a single $(n-1)$-manifold. To see this let $\mathcal{M}$ be one of the components of $\Gamma_{c}$. By the Jordan-Brouwer type separation theorem for manifolds (see the comments after Theorem 4.4), we find that $\mathbb{R}^{n} \backslash \mathcal{M}$ consists of exactly two domains. We denote by $\mathbb{V}$ the bounded component of $\mathbb{R}^{n} \backslash \mathcal{M}$ and note that $\partial \mathbb{V}=\mathcal{M}$, so $U_{\text {ləv }} \equiv c$. It then follows that $\mathbb{V}$ cannot lie entirely in $\Omega \backslash \mathcal{M}$, for otherwise the constant function $c$ on $\mathbb{V}$ would give us the smallest energy. But this contradicts $c$ being a regular value. Since $\Gamma_{0}$ is connected and $\Gamma_{c} \cap \mathcal{M}=\emptyset$, it must lie entirely in one of the components $\mathbb{V}$ or $\mathbb{R}^{n} \backslash \mathbb{V}$. Obviously, $\Gamma_{0} \nsubseteq \mathbb{V}$, because $\Gamma_{0}$ is unbounded. Hence $\Gamma_{0} \subset \mathbb{R}^{n} \backslash \mathbb{V}$. On the other hand, since 
$\mathbb{V} \nsubseteq \Omega \backslash \mathcal{M}=\mathbb{R}^{n} \backslash\left(\Gamma_{0} \cup \Upsilon \cup \mathcal{M}\right)$, we then see that

$$
\mathbb{V} \cap\left(\Gamma_{0} \cup \Upsilon \cup \mathcal{M}\right) \neq \emptyset
$$

where $\mathbb{V} \cap \Gamma_{0}=\emptyset$ and $\mathbb{V} \cap \mathcal{M}=\emptyset$. This yields $\mathbb{V} \cap \Upsilon \neq \emptyset$. Since $\Upsilon$ is connected and disjoint with $\mathcal{M}$, we conclude that $\Upsilon \subset \mathbb{V}$.

Now, suppose that there exists another component $\mathcal{M}^{\prime}$ of the level set $\Gamma_{c}$. Then, by the same reasoning, the bounded component of $\mathbb{R}^{n} \backslash \mathcal{M}^{\prime}$, denoted by $\mathbb{V}^{\prime}$, would contain $\Upsilon$. In particular $\mathbb{V}^{\prime} \supset \mathbb{V}$, because $\mathbb{V}$ is connected and disjoint with $\mathcal{M}^{\prime}$. Similarly, by symmetry of reasonings, we find that $\mathbb{V} \supset \mathbb{V}^{\prime}$, so $\mathbb{V}=\mathbb{V}^{\prime}$. We just proved the following:

Lemma 5.1. Every regular level set $\Gamma_{c}, 0<c<1$, of the potential function $U$ is a single $\mathscr{C}^{\infty}$-smooth $(n-1)$-manifold which separates $\Upsilon$ from $\Omega_{0}$. The component inside $\Gamma_{c}$ is described precisely as $\mathbb{V}=\left\{y \in \mathbb{R}^{n} ; U(y)>c\right\}$.

\section{A COUnterexample to THE CONTINuOUs EXTEnsion AND THE PROOF OF THEOREM 1.3}

We begin with an example where continuous extension is impossible, due mainly to the geometric irregularity of $\partial \mathbb{Y}$.

6.1. A counterexample (volcanic hills of finite energy). We take for a domain $\mathbb{X}$ the lower half-ball in $\mathbb{R}^{n}$ :

$$
\mathbb{X}=\mathbb{U}_{R}=\left\{x=\left(x_{1}, \ldots, x_{n}\right) ;|x|<R \text { and } x_{n}<0\right\} \subset \mathbb{R}_{-}^{n} .
$$

In fact, $\mathbb{X}$ can be any bounded domain in $\mathbb{R}_{-}^{n}$ whose flat top of the boundary $\overline{\mathbb{X}} \cap \mathbb{R}^{n-1}$ has nonempty interior in $\mathbb{R}^{n-1}$. Let $\mathbb{U}=\mathbb{U}(a, r)$ be any lower half-ball in $\mathbb{X}$ with radius $r$ centered at a point $a=\left(a_{1}, \ldots, a_{n-1}, 0\right) \in \overline{\mathbb{X}} \cap \mathbb{R}^{n-1}$. Let us interpret $\mathbb{U}$ as a chamber of lava which is going to erupt from below the surface of $\overline{\mathbb{X}} \cap \mathbb{R}^{n-1}$, cool and harden into a volcanic hill. For computational simplicity we take $a=0$.

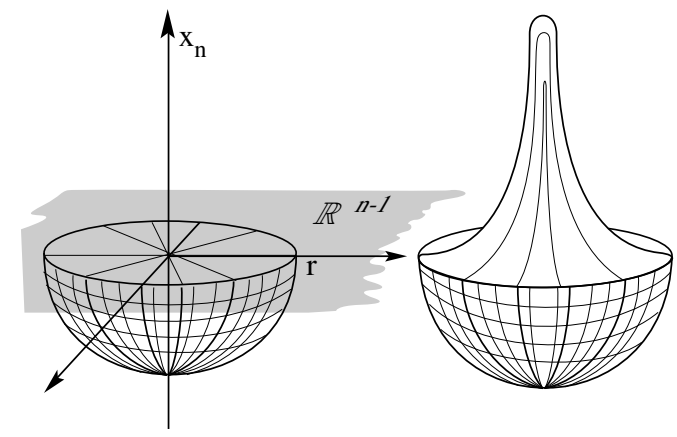

Figure 3. The chamber of lava below the surface and the volcanic hill.

The emission of lava is expressed by a map $\varphi: \overline{\mathbb{U}} \rightarrow \mathbb{R}^{n}$ given by the following equation:

$$
\varphi(x)=\left(x_{1}, \ldots, x_{n-1}, x_{n}+\epsilon(x)\right), \quad x \in \overline{\mathbb{U}}
$$


where

$$
\epsilon(x)=1-\frac{\log (1+\lambda|x|)}{\log (1+\lambda r)} \geqslant 0 .
$$

Here $\lambda$ can be any positive parameter to be chosen depending on the energy we want to designate for the specific amount of lava to be lifted up. In any case, for each $\lambda$, the lava flow always reaches the top at $\varphi(0)=(0, \ldots, 0,1)$. Note that $\epsilon(x)$ is strictly increasing with respect to the variable $x_{n} \in[-r, 0]$. Therefore, $\varphi$ is a continuous injection (homeomorphism) of $\mathbb{U}$ onto a domain in $\mathbb{R}^{n}$. Moreover, $\varphi=i d$ on the lower round part of $\partial \mathbb{U}$, because $\epsilon(x)=0$ for $|x|=r$. The flat top part of $\partial \mathbb{U}$, however, is lifted up into a spike-like formation. We regard $\epsilon$ as a small perturbation of the identity map. Indeed, we have

$$
\begin{aligned}
\mathcal{E}[\varphi-i d] & =\int_{\mathbb{U}}|\nabla \epsilon(x)|^{n} \mathrm{~d} x=\frac{1}{2 \log ^{n}(1+\lambda r)} \int_{|x| \leqslant r} \frac{\lambda^{n} \mathrm{~d} x}{(1+\lambda|x|)^{n}} \\
& =\frac{\omega_{n-1}}{2 \log ^{n}(1+\lambda r)} \int_{0}^{\lambda r} \frac{s^{n-1} \mathrm{~d} s}{(1+s)^{n}} \leqslant \frac{\omega_{n-1}}{2 \log ^{n}(1+\lambda r)} \rightarrow 0
\end{aligned}
$$

as $\lambda r \rightarrow \infty$. We now consider a sequence of points $a_{i} \in \mathbb{R}^{n-1}$ on the upper flat part of $\partial \mathbb{X}$ converging to a point $a \in \overline{\mathbb{X}} \cap \mathbb{R}^{n-1}$. Call $a_{i}$ the epicenters of volcanic hills. The radii $r_{i}<R-\left|a_{i}\right|$ are chosen to be small enough so that the lava chambers $\mathbb{U}_{i}=\mathbb{U}\left(a_{i}, r_{i}\right) \subset \mathbb{X}$ are disjoint. Precisely,

$$
r_{i}+r_{j}<\left|a_{i}-a_{j}\right| \quad \text { for all } i, j=1,2, \ldots
$$

Upon eruption (from each chamber) there will appear a sequence of volcanoes over $\mathbb{X} \cap \mathbb{R}^{n-1}$. The emergent region will be denoted by $\mathbb{Y}$. Precisely, $\mathbb{Y}$ is the image of $\mathbb{X}$ under a homeomorphism $h: \mathbb{X} \stackrel{\text { onto }}{\longrightarrow} \mathbb{Y}$ defined by

$$
h(x)=x+\sum_{i=1}^{\infty} \epsilon_{i}(x) e_{n}, \quad e_{n}=(0, \ldots, 0,1),
$$

where

$$
\epsilon_{i}(x)= \begin{cases}1-\frac{\log \left(1+\lambda_{i}\left|x-a_{i}\right|\right)}{\log \left(1+\lambda_{i} r_{i}\right)} & \text { for } x \in \mathbb{U}_{i} \\ 0 & \text { otherwise. }\end{cases}
$$

Finally, we choose the parameters $\lambda_{i}>0$ to ensure that $h$ has finite conformal energy. Indeed we have

$$
\mathcal{E}[h-i d]=\sum_{i=1}^{\infty} \int_{\mathbb{U}_{i}}\left|\nabla \epsilon_{i}(x)\right|^{n} \mathrm{~d} x \leqslant \frac{\omega_{n-1}}{2} \sum_{i=1}^{\infty} \log ^{1-n}\left(1+\lambda_{i} r_{i}\right) .
$$

This energy can be made as small as desired by taking sufficiently large $\lambda_{i}$ 's; see Figure 4.

Clearly $h: \mathbb{X} \stackrel{\text { onto }}{\longrightarrow} \mathbb{Y}$ admits no continuous extension to $\overline{\mathbb{X}}$ because $\operatorname{diam} h\left(\mathbb{U}_{i}\right)>1$, while $\operatorname{diam}\left(\mathbb{U}_{i}\right)=2 r_{i} \rightarrow 0$. Actually, no homeomorphism $g: \mathbb{X} \stackrel{\text { onto }}{\longrightarrow} \mathbb{Y}$ admits a continuous extension to $\overline{\mathbb{X}}$. This is because the entire interval $\mathbb{I}=\left\{a+t e_{n} ; 0<\right.$ $t \leqslant 1\} \subset \partial \mathbb{Y}$ consists of inaccessible boundary points. Specifically, there is no continuous path $\gamma:[0,1] \subset \mathbb{R}^{n}$, such that $\gamma(0) \in \mathbb{I}$ and $\gamma(0,1] \subset \mathbb{Y}$. Yet, every point $x \in \partial \mathbb{X}$ can be connected with an interior point of $\mathbb{X}$ by a straight segment. 


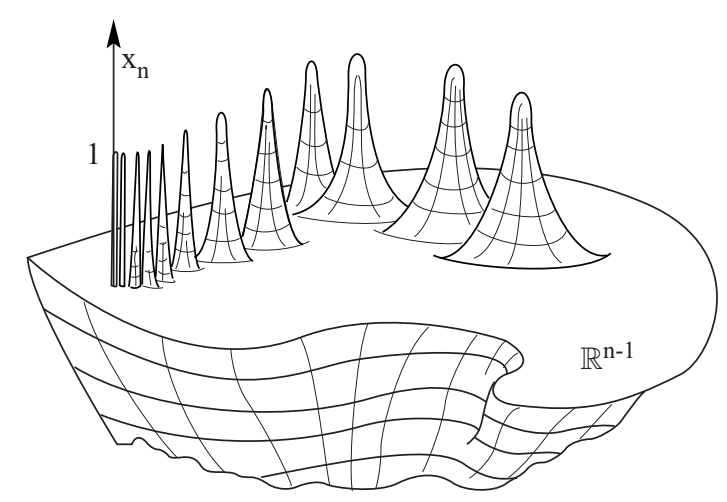

FIGURE 4. Volcanic hills converging to inaccessible boundary.

6.2. Proof of Theorem 1.3. In order to obtain continuity up to the closure of $\mathbb{X}$, we must assume some geometric regularity of $\partial \mathbb{Y}$. Note that in the example of volcanic hills, the boundary of $\mathbb{Y}$ was neither a neighborhood retract, nor was it locally connected. We shall establish Theorem 1.3 if we prove the following:

Proposition 6.1. Suppose that for some $k=0,1, \ldots, \ell$ the component $\partial \mathbb{X}_{k}$ is quasiconformally flat and that $\partial \mathbb{Y}_{k}$ is a neighborhood retract. Then every $h \in \mathcal{E}(\mathbb{X}, \mathbb{Y})$ extends to a continuous map $h: \mathbb{X} \cup \partial \mathbb{X}_{k} \stackrel{\text { onto }}{\longrightarrow} \mathbb{Y} \cup \partial \mathbb{Y}_{k}$.

Before embarking on the proof, let us make a few comments.

The boundary component $\partial \mathbb{X}_{k}$ is quasiconformaly flat if every point in $\partial \mathbb{X}_{k}$ has a neighborhood $\mathbb{U} \subset \mathbb{R}^{n}$ and a quasiconformal map $g: \mathbb{U} \cap \mathbb{X} \rightarrow \mathbb{B}^{+}=\{x=$ $\left.\left(x_{1}, \ldots, x_{n}\right) \in \mathbb{B}, x_{n}>0\right\}$ which extends homeomorphically to $\mathbb{U}$. On the other hand, $\partial \mathbb{Y}_{k}$ is said to be a neighborhood retract if $\partial \mathbb{Y}_{k}$ has a neighborhood $\mathbb{V} \subset \mathbb{R}^{n}$ and a continuous map $\chi: \mathbb{V} \stackrel{\text { onto }}{\longrightarrow} \mathbb{Y}_{k}$ which is the identity on $\partial \mathbb{Y}_{k}$.

Every component of $\partial \mathbb{Y}$ which is locally a graph is a neighborhood retract; see Corollary 10.2. One more point to emphasize is that a continuum in $\mathbb{R}^{n}$ which is a neighborhood retract is locally connected. The converse is not always true, as shown by the Hawaiian earring. The fundamental role played by the quasiconformal mapping $g: \mathbb{U} \cap \mathbb{X} \rightarrow \mathbb{B}^{+}$is that, upon using it as a change of variables, we get a new mapping with finite conformal energy. This will reduce us to the case when $\partial \mathbb{X}_{k}$ is flat near the point at which continuity of $h$ is in question. By contrast, local analysis at a given point of $\partial \mathbb{Y}_{k}$ is impossible because (a priori) the values of $h$ may scatter along all the boundary components of $\partial \mathbb{Y}_{k}$.

Proof of Proposition 6.1. It suffices to show that $h: \mathbb{X} \stackrel{\text { onto }}{\longrightarrow} \mathbb{Y}$ has a limit at every point $x_{0} \in \partial \mathbb{X}_{k}$. Fix such a point. With the aid of a quasiconformal change of variables near $x_{0}$, we may assume that $x_{0}$ is the origin of $\mathbb{R}^{n}$ and that $\partial \mathbb{X}_{k}$ contains the $(n-1)$-disk. That is,

$$
\mathbb{B} \cap \mathbb{R}^{n-1}=\left\{\left(x_{1}, \ldots, x_{n}\right) ; x_{n}=0, x_{1}^{2}+\cdots+x_{n-1}^{2}<1\right\} \subset \partial \mathbb{X}_{k} .
$$

We may further assume that $\mathbb{B}^{+} \subset \mathbb{X}$ and $\mathbb{B}^{-} \subset \mathbb{R}^{n} \backslash \overline{\mathbb{X}}$. The existence of the limit $\lim _{x \rightarrow x_{0}} h(x)$, where $x \in \mathbb{X}$, reduces to showing that

$$
\lim _{r \rightarrow 0} \operatorname{diam} h\left(\mathbb{B}_{r}^{+}\right)=0, \quad \mathbb{B}_{r}^{+}=\mathbb{B}^{+} \cap \mathbb{B}_{r} .
$$


As a first step we extend $h=h\left(x_{1}, \ldots, x_{n-1}, x_{n}\right)$ to $\mathbb{B} \backslash \mathbb{R}^{n-1}=\mathbb{B}^{+} \cup \mathbb{B}^{-}$via even reflection. We shall, by convenient abuse of notation, continue to denote it by the same symbol:

$$
h\left(x_{1}, \ldots, x_{n-1}, x_{n}\right)= \begin{cases}h\left(x_{1}, \ldots, x_{n-1}, x_{n}\right) & \text { if } x_{n}>0 \text { and }|x|<1, x \in \mathbb{B}^{+} \\ h\left(x_{1}, \ldots, x_{n-1},-x_{n}\right) & \text { if } x_{n}<0 \text { and }|x|<1, x \in \mathbb{B}^{-}\end{cases}
$$

Of course, injectivity of $h: \mathbb{B} \backslash \mathbb{R}^{n-1} \rightarrow \mathbb{R}^{n}$ is lost. It is a well-known fact, however, that such an extension has a representative in the Sobolev space $\mathscr{W}^{1, n}\left(\mathbb{B}, \mathbb{R}^{n}\right)$. In general, functions in $\mathscr{W}^{1, n}(\mathbb{B})$ need not be continuous. Nevertheless, they have arbitrarily small oscillations around every point. Precisely, upon a suitable choice of the representative, $h$ becomes continuous on almost every sphere $\mathbb{S}_{r}=\{x ; \quad|x|=$ $r\}, 0<r \leqslant 1$. Moreover, for some $r_{\nu} \rightarrow 0$ we have

$$
\lim _{r_{\nu} \rightarrow 0} \operatorname{diam} h\left(\mathcal{S}_{\nu}\right)=0, \quad \mathcal{S}_{\nu}=\mathbb{S}_{r_{\nu}} ;
$$

see Lemma 3.3. Recall that we have a retraction $\chi: \mathbb{V} \stackrel{\text { onto }}{\longrightarrow} \partial \mathbb{Y}_{k}$ of $\mathbb{V}$ onto $\partial \mathbb{Y}_{k}$. We restrict our considerations to a sufficiently small ball $\mathbb{B}_{r}$, with $0<r<r_{0} \leqslant 1$, so that

$$
h\left(\mathbb{B}_{r}^{+} \cup \mathbb{B}_{r}^{-}\right)=h\left(\mathbb{B}_{r}^{+}\right) \subset \mathbb{V}, \quad \text { for all } 0<r<r_{0} .
$$

The existence of $r_{0}$ is clear because $h$, being a homeomorphism on $\mathbb{X}$, takes points near $\partial \mathbb{X}_{k}$ uniformly close to $\partial \mathbb{Y}_{k}$. Now the Strong Separation Theorem, Theorem 4.6. comes into play. In this theorem we take for $\mathcal{S}$ the sphere $\mathcal{S}_{\nu}$, while for $\mathfrak{X}$ the lower hemisphere $\mathfrak{X}=\left\{\left(x_{1}, \ldots, x_{n}\right) ; x_{n} \leqslant 0\right.$ and $\left.|x|=r_{\nu}\right\}$. We then define a continuous map $\Phi: \mathcal{S}_{\nu} \rightarrow \mathbb{R}^{n}$ by the rule

$$
\Phi(x)= \begin{cases}h(x) & \text { if } x \in \mathcal{S}_{\nu} \cap \mathbb{X} \\ \chi(h(x)) \subset \partial \mathbb{Y}_{k} & \text { if } x \in \mathcal{S}_{\nu} \cap \mathbb{R}^{n} \backslash \mathbb{X} .\end{cases}
$$

Note that $\Phi$ is injective on $\mathcal{S}_{\nu} \backslash \mathfrak{X}$. Moreover, the sets $\Phi(\mathfrak{X}) \subset \partial \mathbb{Y}_{k}$ and $\Phi\left(\mathcal{S}_{\nu} \backslash \mathfrak{X}\right) \subset \mathbb{Y}$ are disjoint. By virtue of Theorem 4.6 the image $\Phi\left(\mathcal{S}_{\nu}\right)$ disconnects the space. Let us estimate its diameter

$$
\operatorname{diam} \Phi\left(\mathcal{S}_{\nu}\right) \leqslant \operatorname{diam} h\left(\mathcal{S}_{\nu}\right)+\operatorname{diam} \chi\left(h\left(\mathcal{S}_{\nu}\right)\right)
$$

because $h\left(\mathcal{S}_{\nu}\right) \cap \chi\left(h\left(\mathcal{S}_{\nu}\right)\right)=h(\mathfrak{X}) \neq \emptyset$. This, in view of (45) and the uniform continuity of $\chi$, shows that $\operatorname{diam} \Phi\left(\mathcal{S}_{\nu}\right) \rightarrow 0$. Now, (44) will be established once we show that $h\left(\mathbb{B}_{r}^{+}\right)$lies in a bounded component of $\mathbb{R}^{n} \backslash \Phi\left(\mathcal{S}_{\nu}\right)$, for $r<r_{\nu}$. First we observe that the connected region $h\left(\mathbb{B}_{r_{\nu}}^{+}\right) \subset \mathbb{Y}$ does not intersect $\Phi\left(\mathcal{S}_{\nu}\right)$; it must lie entirely in one and only one component of $\mathbb{R}^{n} \backslash \Phi\left(\mathcal{S}_{\nu}\right)$. Similarly, the remaining connected region $h\left(\mathbb{X} \backslash \mathbb{B}_{r_{\nu}}^{+}\right)=\mathbb{Y} \backslash h\left(\mathbb{B}_{r_{\nu}}^{+}\right)$lies in one and only one component of $\mathbb{R}^{n} \backslash \Phi\left(\mathcal{S}_{\nu}\right)$. Taking $r_{\nu}$ sufficiently small we see that the set $h\left(\mathbb{X} \backslash \mathbb{B}_{r_{\nu}}^{+}\right)$is too large to fit in any bounded component of $\mathbb{R}^{n} \backslash \Phi\left(\mathcal{S}_{\nu}\right)$. Therefore, $h\left(\mathbb{X} \backslash \mathbb{B}_{r_{\nu}}^{+}\right)$must lie in an unbounded component of $\mathbb{R}^{n} \backslash \Phi\left(\mathcal{S}_{\nu}\right)$. At this point in our argument the second statement of Lafont's Theorem 4.6 proves useful. Accordingly, to every point $p \in \Phi\left(\mathcal{S}_{\nu} \backslash \mathfrak{X}\right)$ there correspond precisely two connected components of $\mathbb{R}^{n} \backslash$ $\Phi\left(\mathcal{S}_{\nu}\right)$ which contain $p$ in their closure. On the other hand such a $p$ belongs to $\overline{h\left(\mathbb{B}_{r_{\nu}}^{+}\right)} \cap \overline{h\left(\mathbb{X} \backslash \mathbb{B}_{r_{\nu}}^{+}\right)}$and is an interior point of $\overline{h\left(\mathbb{B}_{r_{\nu}}^{+}\right)} \cup \overline{h\left(\mathbb{X} \backslash \mathbb{B}_{r_{\nu}}^{+}\right)}$. This means that $h\left(\mathbb{B}_{r_{\nu}}^{+}\right)$and $h\left(\mathbb{X} \backslash \mathbb{B}_{r_{\nu}}^{+}\right)$are the only components which contain $p$ in their closer. In particular, these are different components of $\mathbb{R}^{n} \backslash \Phi\left(\mathcal{S}_{\nu}\right)$. Finally, there is only one 
unbounded component. Therefore the set $h\left(\mathbb{B}_{r_{\nu}}^{+}\right)$lies in a bounded component of $\mathbb{R}^{n} \backslash \Phi\left(\mathcal{S}_{\nu}\right)$, as does its subset $h\left(\mathbb{B}_{r}^{+}\right)$for $0<r \leqslant r_{\nu}$, as desired.

Remark 6.1. The reader may wish to observe that we actually proved more. Let $\mathfrak{X}$ be a quasiconformal crack in $\mathbb{X}$ of dimension $n-1$. That is, each point in $\mathfrak{X}$ has a neighborhood $\mathbb{U}$ and a quasiconformal map $g: \mathbb{U} \rightarrow \mathbb{B}$ such that $g(\mathbb{U} \cap \mathfrak{X})=$ $g(\mathbb{U}) \cap \mathbb{R}^{n-1}$. On the other hand, let $\Upsilon \subset \mathbb{Y}$ be a neighborhood retract. We view $\mathfrak{X}$ and $\Upsilon$ as boundary components of $\mathbb{X} \backslash \mathfrak{X}$ and $\mathbb{Y} \backslash \Upsilon$ and $h \in \mathcal{E}(\mathbb{X} \backslash \mathfrak{X}, \mathbb{Y} \backslash \Upsilon)$. Obviously, $\mathfrak{X}$ disconnects $\mathbb{U}$. What we really proved is that $h$ extends continuously to the closure of every component of $\mathbb{U} \backslash \mathfrak{X}$, but not necessarily to $\mathbb{U}$. The following example illustrate this phenomenon.

Example 6.1 (A cavity). Let $\mathfrak{X}$ be an $(n-1)$-dimensional disk in $\mathbb{R}^{n}=\mathbb{R}^{n-1} \times \mathbb{R}$ of the form $\mathfrak{X}=\{(w, 0):|w| \leqslant 1\}$. Define a $\mathscr{C}^{\infty}$-smooth homeomorphism $h$ : $\mathbb{R}^{n} \backslash \mathfrak{X} \rightarrow \mathbb{R}^{n}$ by the rule

$$
h(x)=h(w, t)= \begin{cases}(w, t+\tau(w)) & \text { if } \quad t>0, \\ (w, t) & \text { if } \quad t=0 \text { and }|w|>1,\end{cases}
$$

where $\tau \in \mathscr{C}_{\circ}^{\infty}\left(\mathbb{R}^{n-1}\right)$ is positive for $|w|<1$ and vanishes for $|w| \geqslant 1$. This map has a bounded gradient and yet makes a hole out of the disk $\mathfrak{X}$.

However, if the crack $\mathfrak{X} \subset \mathbb{X}$ has dimension less than $n-1$, then we have the following result 33 .

Theorem 6.1. Suppose $h \in \mathscr{W}_{\text {loc }}^{1, n}\left(\mathbb{X}, \mathbb{R}^{n}\right)$ is a continuous injection outside a closed set $\mathfrak{X} \subset \mathbb{R}^{n}$, with $\operatorname{dim} \mathfrak{X} \leqslant n-2$. Then $h$ extends continuously from $\mathbb{X} \backslash \mathfrak{X}$ to $\mathbb{X}$.

\section{Estimates OF THE DistanCE TO THE BOUNDARY}

As has been suggested by Definition 1.1, the notion of a Euclidean diameter of a continuum $\mathfrak{X}$ in a domain $\mathbb{X} \subset \mathbb{R}^{n}$ is not best suited for our purpose.

7.1. The essential diameter. Let $\mathcal{H}(\mathbb{X}, \mathbb{X})$ denote the collection of all homeomorphisms $\varphi: \mathbb{X} \stackrel{\text { onto }}{\longrightarrow} \mathbb{X}$ (topological automorphisms) such that $\varphi\left\{\partial \mathbb{X}_{i}\right\}=\partial \mathbb{X}_{i}$, $i=0,1, \ldots, \ell$.

Definition 7.1. The essential diameter of a continuum $\mathfrak{X} \subset \mathbb{X}$ relative to $\mathbb{X}$ is defined and denoted by

$$
\operatorname{diam}(\mathfrak{X}, \mathbb{X})=\inf \{\operatorname{diam} \varphi(\mathfrak{X}) ; \varphi \in \mathcal{H}(\mathbb{X}, \mathbb{X})\} .
$$

As an illustration, every continuum $\mathfrak{X}$ in a ball $\mathbb{B} \subset \mathbb{R}^{n}$ can be compressed by a homeomorphism in $\mathcal{H}(\mathbb{X}, \mathbb{X})$ to a set of arbitrarily small diameter. Thus $\operatorname{diam}(\mathfrak{X}, \mathbb{X})=0$. On the other hand, consider a circle $\mathfrak{X} \subset \mathbb{R}^{n}$ of radius $R$ and the torus $\mathbb{X}=\left\{x \in \mathbb{R}^{n} ; \operatorname{dist}(x, \mathfrak{X})<r\right\}, 0<r<R$. The essential diameter of $\mathfrak{X}$ relative to $\mathbb{X}$ can easily be computed as

$$
\operatorname{diam}(\mathfrak{X}, \mathbb{X})=2(R-r) .
$$

Let us also note that every continuum $\mathfrak{X} \subset \mathbb{X}$ which separates the outer component $\mathbb{X}_{0}$ of $\mathbb{R}^{n} \backslash \mathbb{X}$ from an inner component $\mathbb{X}_{i}, i=1,2, \ldots, \ell$, has the essential diameter not smaller than $\operatorname{diam} \mathbb{X}_{i}$,

$$
\operatorname{diam}(\mathfrak{X}, \mathbb{X}) \geqslant \operatorname{diam} \mathbb{X}_{i}
$$


In particular, $\operatorname{diam}(\mathfrak{X}, \mathbb{X})$ is positive if the component $\mathbb{X}_{i}$ of $\mathbb{R}^{n} \backslash \mathbb{X}$ is not a single point (not a puncture in $\mathbb{R}^{n}$ ). We also emphasize that if $\mathfrak{X}$ separates $\mathbb{X}_{0}$ from two punctures $\mathbb{X}_{i}=\left\{a_{i}\right\}$ and $\mathbb{X}_{j}=\left\{a_{j}\right\}$, then

$$
\operatorname{diam}(\mathfrak{X}, \mathbb{X}) \geqslant\left|a_{i}-a_{j}\right| .
$$

It is now well to point out that a domain $\mathbb{X} \subset \mathbb{R}^{n}$ is incompressible if it contains a continuum $\mathfrak{X} \subset \mathbb{X}$ such that $\operatorname{diam}(\mathfrak{X}, \mathbb{X})>0$.

For many analytic-geometric questions this property of $\mathbb{X}$ well compensates the lack of existence of the inner boundaries. A little caution should be exercised since domains of the same topological type as torus may be compressible, for example $\mathbb{B} \backslash \mathbb{R}^{n-2}$.

7.2. The modulus of the distance to $\partial \mathbb{Y}_{i}$. Recall the family $\mathcal{E}(\mathbb{X}, \mathbb{Y})$ of homemorphisms $h: \mathbb{X} \stackrel{\text { onto }}{\longrightarrow} \mathbb{Y}, h\left\{\partial \mathbb{X}_{i}\right\}=\left\{\partial \mathbb{Y}_{i}\right\}, i=0,1, \ldots, \ell$, with finite conformal energy

$$
\mathcal{E}[h]=\int_{\mathbb{X}}|D h|^{n}<\infty .
$$

Here, we are concerned with uniform estimates of the distance of $h(x)$ to $\partial \mathbb{Y}_{i}$ as $x$ approaches $\partial \mathbb{X}_{i}$, in relation to the energy of $h$. Thus, we introduce the moduli of the distance functions defined for $x \in \mathbb{X}$ by the rules.

$$
\eta_{i}(x)=\sup _{h \in \mathcal{E}(\mathbb{X}, \mathbb{Y})} \frac{\operatorname{dist}^{n}\left[h(x), \mathbb{Y}_{i}\right]}{\int_{\mathbb{X}}|D h|^{n}}, \quad i=0,1, \ldots, \ell,
$$

and

$$
\eta(x)=\eta_{\mathbb{X}, \mathbb{Y}}(x)=\sup _{h \in \mathcal{E}(\mathbb{X}, \mathbb{Y})} \frac{\operatorname{dist}^{n}[h(x), \partial \mathbb{Y}]}{\int_{\mathbb{X}}|D h|^{n}}=\max _{0 \leqslant i \leqslant \ell} \eta_{i}(x) .
$$

Theorem 7.1. Suppose that $\mathbb{X}$ has at least two boundary components or is incompressible. Then for each component $\mathbb{X}_{i}$ of $\mathbb{R}^{n} \backslash \mathbb{X}, i=0,1, \ldots, \ell$, we have

$$
\lim _{x \rightarrow \mathbb{X}_{i}} \eta_{i}(x)=0, \quad \text { so } \quad \lim _{x \rightarrow \partial \mathbb{X}} \eta_{\mathbb{X}, \mathbb{Y}}(x)=0 .
$$

7.3. Proof of Theorem 7.1. There are several cases to consider. First we handle the inner components, $1 \leqslant i \leqslant \ell$.

7.3.1. Estimates near the inner component. There are two types of inner components.

Case 1. The component $\mathbb{X}_{i}$ is a single point, say $\mathbb{X}_{i}=\{a\}$.

It is not difficult to see that the corresponding component $\mathbb{Y}_{i}=h\left\{\mathbb{X}_{i}\right\}$ is also a single point, say $\mathbb{Y}_{i}=\{b\}$. Moreover, every $h \in \mathcal{E}(\mathbb{X}, \mathbb{Y})$ extends to a homeomorphism $h: \mathbb{X} \cup \mathbb{X}_{i} \stackrel{\text { onto }}{\longrightarrow} \mathbb{Y} \cup \mathbb{Y}_{i}$; see Theorem 3.1. We fix the parameter $d>0$ so that the ball $2 \mathbb{B}=\mathbb{B}(a, 2 d)$ lies in $\mathbb{X} \cup \mathbb{X}_{i}$. By Lemma 3.1 we obtain

$$
|h(x)-h(a)|^{n} \leqslant \frac{C_{n} \int_{2 \mathbb{B}}|D h|^{n}}{\log \left(e+\frac{d}{|x-a|}\right)} \quad \text { for } \quad x \in \mathbb{B}=\mathbb{B}(a, d) .
$$

Thus $\lim _{x \rightarrow a} \eta_{i}(x)=0$, as claimed. 
Remark 7.1. We can state the result as

$$
\operatorname{dist}^{n}\left[h(x), \partial \mathbb{Y}_{i}\right] \leqslant \frac{C_{n}(\mathbb{X}, \mathbb{Y}) \int_{\mathbb{X}}|D h|^{n}}{\log \left(e+\frac{\operatorname{diam} \mathbb{X}}{\operatorname{dist}\left(x, \partial \mathbb{X}_{i}\right)}\right)} .
$$

This estimate will be generalized later for monotone components of $\partial \mathbb{Y}$, which will receive detailed consideration in Section 8.3 ,

The next case is the essence of Theorem 7.1. Its proof is the key to other cases, so we give all details.

Case 2. Suppose $\mathbb{X}_{i}$ is an inner component of $\mathbb{R}^{n} \backslash \mathbb{X}$ with positive diameter, say

$$
\operatorname{diam} \mathbb{X}_{i}=2 d>0 .
$$

We emphasize that the corresponding component $\mathbb{Y}_{i}$ may still be a single point. The general idea of the proof amounts to introducing a potential function in the target space. Our choice of the potential function will depend on the regularity of $\partial \mathbb{Y}_{i}$. Specific estimates will be obtained if the target domain $\mathbb{Y}$ is monotone near $\partial \mathbb{Y}_{i}$; see Definition 8.1. However, in this section, we do not deal with quantitative estimates, and that is why Theorem 7.1 requires no regularity of $\partial \mathbb{Y}_{i}$.

Let us begin with the set $\Omega=\mathbb{R}^{n} \backslash \mathbb{Y}_{0}=\mathbb{Y} \cup \mathbb{Y}_{1} \cup \cdots \cup \mathbb{Y}_{\ell}$. By Lemma 4.3 this is a bounded domain. Obviously its complement $\mathbb{R}^{n} \backslash \Omega=\mathbb{Y}_{0}$ is also connected. We may, therefore, consider the potential function $U$ for the condenser $\left(\mathbb{Y}_{i}, \Omega\right)$ as discussed in Section 55 see Lemma 5.1 with $\mathbb{Y}_{i}$ in place of $\Upsilon$. By Sard's theorem we see that almost every level set $\Gamma_{c}, 0<c<1$, is a smooth $(n-1)$-manifold which separates $\mathbb{Y}_{i}$ from $\mathbb{Y}_{0}$. Even more, if $c$ is sufficiently close to 1 , then $\Gamma_{c}$ separates $\mathbb{Y}_{i}$ from all other components of $\mathbb{R}^{n} \backslash \mathbb{Y}$. In particular, the bounded component of $\mathbb{R}^{n} \backslash \Gamma_{c}$ is contained in $\mathbb{Y} \cup \mathbb{Y}_{i}$. We fix such a value $c$ and will only consider the level sets $\Gamma_{t}$ with $c \leqslant t \leqslant 1$. Let us truncate the potential function

$$
U_{c}=U_{c}(y) \stackrel{\text { def }}{=} \max \{c, U(y)\}, \quad y \in \mathbb{R}^{n} .
$$

As a first step we observe that if $f: \mathbb{Y} \stackrel{\text { onto }}{\longrightarrow} \mathbb{X}$ is a homeomorphism with $f\left\{\partial \mathbb{Y}_{i}\right\}=$ $\partial \mathbb{X}_{i}$, for $i=0,1, \ldots, \ell$, then

Lemma 7.1. For every regular level set $\Gamma_{t}$ of $U, 0<t<1$, it holds that

$$
\operatorname{diam} f\left(\Gamma_{t}\right) \geqslant 2 d .
$$

Proof. By Theorem 4.6 the set $\mathbb{R}^{n} \backslash f\left(\Gamma_{t}\right)$ consists of exactly two components. On the other hand $\Gamma_{t}$ separates $\mathbb{Y}_{i}$ from $\mathbb{Y}_{0}$, so $f\left(\Gamma_{t}\right)$ separates $\mathbb{X}_{i}$ from $\mathbb{X}_{0}$. In particular, the bounded component of $\mathbb{R}^{n} \backslash f\left(\Gamma_{t}\right)$ contains $\mathbb{X}_{i}$. This is because $\mathbb{X}_{0}$, being unbounded, does not fit into any bounded component. Hence

$$
\operatorname{diam} f\left(\Gamma_{t}\right) \geqslant \operatorname{diam} \mathbb{X}_{i} \geqslant 2 d
$$

Lemma 7.2. Let $h: \mathbb{X} \stackrel{\text { onto }}{\longrightarrow} \mathbb{Y}$ be a homeomorphism such that $h\left\{\partial \mathbb{X}_{i}\right\}=\partial \mathbb{Y}_{i}$, $i=0,1, \ldots, \ell$. Consider the pullback of $U_{c}$ under $h$, defined by

$$
V(x)=V_{h}(x)= \begin{cases}U_{c}(h(x)) & \text { if } x \in \mathbb{X}, \\ 1 & \text { if } x \in \mathbb{X}_{i}, \\ c & \text { if } x \notin \mathbb{X} \cup \mathbb{X}_{i} .\end{cases}
$$


Then $V$ is continuous in $\mathbb{R}^{n}$ and monotone on every ball $\mathbb{B} \subset \mathbb{R}^{n}$ of diameter not greater than $2 d$. This means that

$$
\min _{\partial \mathbb{Q}} V=\min _{\mathbb{Q}} V \leqslant \max _{\mathbb{Q}} V=\max _{\partial \mathbb{Q}} V
$$

for every compact set $\mathbb{Q} \subset \mathbb{B}$, diam $\mathbb{B} \leqslant 2 d$.

For the proof of this lemma we fix a compact set $\mathbb{Q} \subset \mathbb{B}$ in a ball $\mathbb{B}$ of diameter $2 d$. We aim to show that every value $t \in[c, 1]$ of $V_{\text {le }}$ is actually attained on $\partial \mathbb{Q}$. It involves no loss of generality in assuming that $\mathbb{Q}$ is connected, since otherwise we would restrict $V$ to the component of $\mathbb{Q}$ containing a given point $x$ with $V(x)=t$. We may further assume that $t$ is a regular value of $U$, because such values are dense in $[c, 1]$. Now, the regular level set $\Gamma_{t}=\{y \in \mathbb{Y} ; U(y)=t\}$ contains the point $h(x)$. By Lemma $7.1 \operatorname{diam} f\left(\Gamma_{t}\right) \geqslant 2 d=\operatorname{diam} \mathbb{B}>\operatorname{diam} \mathbb{Q}$. We see that the continuum $f\left(\Gamma_{t}\right)$ is too large to be contained in $\mathbb{Q}$. Thus it must intersect $\partial \mathbb{Q}$, meaning that the value $t$ is attained on $\partial \mathbb{Q}$. This completes the proof of Lemma 7.2 .

Further truncation of $V$ near $\mathbb{X}_{i}$ will be necessary in order to control the gradient of $V$. Given $\alpha \in(c, 1)$ we define

$$
V^{\alpha}(x)=V_{h}^{\alpha}(x)=\min \left\{V_{h}(x), \alpha\right\} .
$$

Lemma 7.3. For every $c<\alpha<1$ the truncated potential $V^{\alpha}(x)$ is still monotone on balls $\mathbb{B} \subset \mathbb{R}^{n}$ of diameter $2 d$.

This fact actually pertains to a truncation of every monotone function $V$; the proof is standard.

We now proceed to the proof of the limits of (56) .

Proof of (56). Suppose that, on the contrary, there exist $\delta>0$, a sequence of points $x_{\nu} \in \mathbb{X}$ converging to $x_{0} \in \mathbb{X}_{i}$, and homeomorphisms $h_{\nu} \in \mathcal{E}(\mathbb{X}, \mathbb{Y})$, such that

$$
\frac{\operatorname{dist}^{n}\left[h_{\nu}\left(x_{\nu}\right), \mathbb{Y}_{i}\right]}{\int_{\mathbb{X}}\left|D h_{\nu}\right|^{n}} \geqslant \delta, \quad \nu=1,2, \ldots
$$

This yields, in particular, that the mappings $h_{\nu}$ have bounded energy. Precisely, we have

$$
\int_{\mathbb{X}}\left|D h_{\nu}\right|^{n} \leqslant \frac{\operatorname{diam}^{n} \mathbb{Y}}{\delta}, \quad \nu=1,2, \ldots
$$

On the other hand,

$$
\operatorname{dist}^{n}\left[h_{\nu}\left(x_{\nu}\right), \mathbb{Y}_{i}\right] \geqslant \delta \int_{\mathbb{X}}\left|D h_{\nu}\right|^{n} \geqslant \delta|\mathbb{Y}|, \quad \nu=1,2, \ldots
$$

Therefore, all points $h_{\nu}\left(x_{\nu}\right) \in \mathbb{Y}$ lie in a compact subset of $\mathbb{Y}$. By virtue of (39) there exists $\alpha \in(c, 1)$, sufficiently close to 1 and independent of $\nu$, such that

$$
c \leqslant U_{c}\left(h_{\nu}\left(x_{\nu}\right)\right) \leqslant \alpha, \quad \nu=1,2, \ldots
$$

We now fix $\alpha$ and select another parameter $\beta$ such that $c<\alpha<\beta<1$. Consider a sequence of functions $V_{\nu}^{\beta}=V_{h_{\nu}}^{\beta}(x)$ defined for $x \in \mathbb{R}^{n}$. The reader may wish to return to formulas (61) and (62) for the definition of $V_{h}^{\beta}$. Clearly, if $c \leqslant U\left(h_{\nu}(x)\right) \leqslant$ $\beta$, then $V_{\nu}^{\beta}(x)=U\left(h_{\nu}(x)\right)$; otherwise $\nabla V_{\nu}^{\beta}(x) \equiv 0$. Hence, we have the following uniform bound of the gradient of $V_{\nu}^{\beta}$ :

$$
\left|\nabla V_{\nu}^{\beta}(x)\right| \leqslant M\left|D h_{\nu}(x)\right|, \quad \text { where } \quad M \stackrel{\text { def }}{=}\|\nabla U\|_{\mathscr{L} \infty(c<U<\beta)} .
$$


Precisely, we have

$$
\int_{\mathbb{X}}\left|\nabla V_{\nu}^{\beta}(x)\right|^{n} \mathrm{~d} x \leqslant \frac{\operatorname{diam}^{n} \mathbb{Y}}{\delta} M^{n}, \quad \text { for } \nu=1,2, \ldots
$$

Since $V_{\nu}^{\beta}$ are monotone on every ball $\mathbb{B}$ of diameter $2 d$, it follows from Lemma 3.1 that

$$
\left|V_{\nu}^{\beta}(a)-V_{\nu}^{\beta}(b)\right|^{n} \leqslant \frac{C_{n} \int_{\mathbb{B}}\left|\nabla V_{\nu}^{\beta}\right|^{n}}{\log \left(e+\frac{d}{|a-b|}\right)} \leqslant \frac{C_{n} M^{n} \operatorname{diam}^{n} \mathbb{Y}}{\delta \log \left(e+\frac{d}{|a-b|}\right)}
$$

whenever $a, b \in \mathbb{R}^{n}$ and $|a-b| \leqslant d$. This shows that the functions in the sequence $\left\{V_{\nu}^{\beta}\right\}_{\nu=1}^{\infty}$ are equicontinuous on $\mathbb{R}^{n}$. By the Ascoli-Arzelá Theorem there is a subsequence, again denoted by $\left\{V_{\nu}^{\beta}\right\}_{\nu=1}^{\infty}$, that converges uniformly on $\mathbb{R}^{n}$ to a continuous function $V^{\beta}=V^{\beta}(x)$,

$$
V_{\nu}^{\beta}(x) \rightrightarrows V^{\beta}(x), \quad x \in \mathbb{R}^{n}
$$

In particular,

$$
V_{\nu}^{\beta}\left(x_{\nu}\right)-V^{\beta}\left(x_{\nu}\right) \rightarrow 0, \quad \text { as } \nu \rightarrow \infty .
$$

Also note that $V_{\nu}^{\beta} \equiv \beta$ on $\mathbb{X}_{i}$ for all $\nu=1,2, \ldots$. Hence $V^{\beta} \equiv \beta$ on $\mathbb{X}_{i}$ as well. It follows from the definition of $V_{\nu}^{\beta}\left(x_{\nu}\right)$ that

$$
V_{\nu}^{\beta}\left(x_{\nu}\right)=U_{c}\left(h_{\nu}\left(x_{\nu}\right)\right) \quad \text { because } U_{c}\left(h_{\nu}\left(x_{\nu}\right)\right)<\beta \text {. }
$$

For this, recall (65) and our choice of $\beta \in(\alpha, 1)$. Finally, passing to the limit as $x_{\nu} \rightarrow x_{0} \in \mathbb{X}_{i}$, we obtain a contradiction:

$$
0=\lim _{\nu \rightarrow \infty}\left[V_{\nu}^{\beta}\left(x_{\nu}\right)-V^{\beta}\left(x_{\nu}\right)\right] \leqslant \alpha-\beta<0 .
$$

Remark 7.2. Let us emphasize that one of the essential points in this proof was that the parameter $d$ in Lemma 7.3 did not depend on $h \in \mathcal{E}(\mathbb{X}, \mathbb{Y})$. However, the same proof still works when $d$ depends on the energy of $h$, but not on the individual member of $\mathcal{E}(\mathbb{X}, \mathbb{Y})$. This is because the energies of $\left\{h_{\nu}\right\}$ stay bounded anyway; see (64). We shall face such a situation in Lemma 7.4 of Section 7.3.2

7.3.2. Estimates near the outer component. We now examine the modulus of the distance to $\mathbb{Y}_{0}$ :

$$
\eta_{0}(x)=\sup _{h \in \mathcal{E}(\mathbb{X}, \mathbb{Y})} \frac{\operatorname{dist}^{n}\left[h(x), \mathbb{Y}_{0}\right]}{\int_{\mathbb{X}}|D h|^{n}} .
$$

The goal is to show that $\lim _{x \rightarrow \mathbb{X}_{0}} \eta_{0}(x)=0$. Our proof is divided into Case 3 and Case 4. In Case 3 we assume that either $\ell=0$ or

$$
\operatorname{diam}\left(\mathbb{X}_{1} \cup \cdots \cup \mathbb{X}_{\ell}\right) \geqslant 2 d>0 .
$$

Recall that in case $\ell=0$ we are given a compact set $\mathfrak{X} \subset \mathbb{X}$ of positive essential diameter. This set will supersede $\mathbb{X}_{1} \cup \cdots \cup \mathbb{X}_{\ell}$. The arguments are much the same as in Case 2.

Case 3. Assume that either $\ell \geqslant 1$ and (67) holds or $\ell=0$ and $\mathbb{X}$ is incompressible. We only emphasize the main points of the proof. Consider a condenser $(\Upsilon, \Omega)$ in the target space where

$$
\Omega=\mathbb{R}^{n} \backslash \mathbb{Y}_{0}=\mathbb{Y} \cup \mathbb{Y}_{1} \cup \cdots \cup \mathbb{Y}_{\ell}
$$

As for $\Upsilon$, we take a continuum which contains $\mathbb{Y}_{1} \cup \cdots \cup \mathbb{Y}_{\ell}$ if $\ell \geqslant 1$. If $\ell=0$, we choose and fix a continuum $\mathfrak{X} \subset \mathbb{X}$ of positive essential diameter and take for 
$\Upsilon$ its image under any (also fixed) homeomorphism $g \in \mathcal{H}(\mathbb{X}, \mathbb{Y})$. In this case the parameter $d$ is the one for which $\operatorname{diam}(\mathfrak{X} \mathbb{X}) \geqslant 2 d$. The associated potential function $U$, exploited effectively in Case 2, also serves well here. However, in order to follow literally the notation and subsequent steps of Case 2, the reader may wish to replace $U$ by $1-U$. Details are left to the reader.

Case 4. We now come to the last step in the proof of Theorem 7.1. That is, when $\mathbb{R}^{n} \backslash \mathbb{X}$ has two components $\mathbb{X}_{0}$ and a single point

$$
\mathbb{R}^{n} \backslash \mathbb{X}=\mathbb{X}_{0} \cup\{a\} .
$$

We know from Theorem 3.1 that $h: \mathbb{X} \stackrel{\text { onto }}{\longrightarrow} \mathbb{Y}$ extends as a homeomorphism $h$ : $\mathbb{X}^{\prime} \stackrel{\text { onto }}{\longrightarrow} \mathbb{Y}^{\prime}$, where $\mathbb{X}^{\prime}=\mathbb{X} \cup\{a\}$ and $\mathbb{Y}^{\prime}=\mathbb{Y} \cup\{b\}, b=\lim _{x \rightarrow a} h(x)$. But it does not mean that we are reduced to Case 3 in which $\partial \mathbb{X}^{\prime}$ has only an outer boundary component. This is because a compact set $\mathfrak{X} \subset \mathbb{X}^{\prime}$ of positive essential diameter is unavailable this time. We compensate it by estimating the size of the image of a neighborhood of $a \in \mathfrak{X}$ under the mappings $h \in\left(\mathbb{X}^{\prime}, \mathbb{Y}^{\prime}\right)$.

Consider a condenser $(\Upsilon, \Omega)$, where $\Omega=\mathbb{Y}^{\prime}$ and $\Upsilon \subset \Omega$ is any (fixed) continuum with nonempty interior, for instance containing some ball $\mathbb{B}(b, \delta)$. The associated potential function $U$, see Lemma 5.1, again works just as well. We propose to replace $U$ by $1-U$ so that the notation and subsequent steps of Case 2 are in force. The regular level sets $\Gamma_{t}=\left\{y \in \mathbb{R}^{n} ; U(y)=t\right\}, 0<t<1$, are single $(n-1)$-manifolds separating $\Upsilon$ from $\partial \mathbb{Y}^{\prime}$. The only point remaining concerns a uniform bound from below of the size of the sets $h^{-1}\left(\Gamma_{t}\right)$.

Lemma 7.4. Fix any positive number $E$. Then for every $h \in \mathcal{E}\left(\mathbb{X}^{\prime}, \mathbb{Y}^{\prime}\right)$, with $h(a)=b$ and $\int_{\mathbb{X}}|D h|^{n} \leqslant E$, and for every regular value $0<t<1$ of the potential function $U$, it holds that

$$
\operatorname{diam} h^{-1}\left(\Gamma_{t}\right) \geqslant 2 d>0, \quad 0<t<1 .
$$

Here $d$ depends only on $\mathbb{X}, \mathbb{Y}$ and $E$.

Let us emphasize that $d$ does not depend on any particular member of the family $\mathcal{E}\left(\mathbb{X}^{\prime}, \mathbb{Y}^{\prime}\right)$, just on the upper bound of the conformal energy; see Remark 7.2 .

Proof. We choose and fix a ball $B(a, 2 R) \subset \mathbb{X}^{\prime}$. Now, consider any map $h \in$ $\mathcal{E}\left(\mathbb{X}^{\prime}, \mathbb{Y}^{\prime}\right)$ with energy $\int_{\mathbb{X}}|D h|^{n} \leqslant E$. By the continuity estimate in Lemma 3.1, we have

$$
|h(x)-h(a)|^{n} \leqslant \frac{C_{n} \cdot E}{\log \left(e+\frac{R}{|x-a|}\right)},
$$

provided $|x-a| \leqslant R$. We now specify the parameter $d$ for (68); namely,

$$
d=\min \{R, r\},
$$

where $r$ is uniquely determined by the equation

$$
\delta^{n}=\frac{C_{n} \cdot E}{\log \left(1+\frac{R}{r}\right)}, \quad \text { recall that } \delta \text { was fixed so } \mathbb{B}(b, \delta) \subset \Upsilon .
$$

For (68), observe that $h^{-1}\left(\Gamma_{t}\right)$ separates $\mathbb{R}^{n}$ into two component. Let $B(a, \epsilon)$ denote the largest ball centered at $a$ which fits inside $h^{-1}\left(\Gamma_{t}\right)$. That is,

$$
\epsilon=\inf \left\{|x-a| ; \quad x \in h^{-1}\left(\Gamma_{t}\right)\right\} .
$$


Therefore, $\operatorname{diam} h^{-1}\left(\Gamma_{t}\right) \geqslant \operatorname{diam} B(a, \epsilon)=2 \epsilon$. Now (68) holds if $\epsilon \geqslant R$. On the other hand, if $0<\epsilon<R$, we may apply inequality (69) with the point $x \in h^{-1}\left(\Gamma_{t}\right)$ for which $\epsilon=|x-a|$. This means that $h(x) \in \Gamma_{t}$ and

$$
|h(x)-h(a)| \geqslant \operatorname{dist}\left\{b, \Gamma_{t}\right\} \geqslant \operatorname{dist}\{b, \partial \Upsilon\}=\delta .
$$

Hence

$$
\delta^{n} \leqslant \frac{C_{n} \cdot E}{\log \left(e+\frac{R}{\epsilon}\right)}<\frac{C_{n} \cdot E}{\log \left(1+\frac{R}{\epsilon}\right)} .
$$

As a consequence of the definition of $r$ at (171) this yields $\epsilon>r$, establishing the inequality (68). All cases have been considered completing the proof of Theorem 7.1.

\section{Estimates NEAR the MONOTONE BOUNDARY}

In this section we continue to study homeomorphisms $h: \mathbb{X} \stackrel{\text { onto }}{\longrightarrow} \mathbb{Y}$ between two bounded domains in $\mathbb{R}^{n}$. Our objective is to examine more precisely how close to $\partial \mathbb{Y}$ is $h(x)$ when $x$ approaches $\partial \mathbb{X}$. As always, we assume that $\mathbb{X}$ and $\mathbb{Y}$ are $(\ell+1)$-connected, $\ell \geqslant 1$. Thus $\mathbb{Y}$ is a bounded connected open set whose complement $\mathbb{R}^{n} \backslash \mathbb{Y}$ consists of $(\ell+1)$-components, denoted by $\mathbb{Y}_{0}, \mathbb{Y}_{1}, \ldots, \mathbb{Y}_{\ell}$. In this numeration $\mathbb{Y}_{0}$ stands for the unbounded component. The boundaries of $\mathbb{Y}_{i}$ are the components of $\partial \mathbb{Y}$, denoted by

$$
\Gamma_{i}=\partial \mathbb{Y}_{i}, \quad i=0,1, \ldots, \ell
$$

\subsection{Monotone domains.}

Definition 8.1. A bounded domain $\mathbb{Y} \subset \mathbb{R}^{n}$ of connectivity $\ell+1$ is said to be monotone near its boundary component $\Gamma_{i} \subset \partial \mathbb{Y}$ if for all sufficiently small $\epsilon>0$ the level sets

$$
\Gamma_{i}^{\epsilon}=\left\{y \in \mathbb{Y} ; \quad \operatorname{dist}\left(y, \Gamma_{i}\right)=\epsilon\right\}
$$

are connected. Furthermore, $\mathbb{Y}$ is monotone if it is bounded and monotone near every boundary component.

Lemma 8.1 (The level sets). Let $\mathbb{Y}$ be a monotone domain and $\mathbb{G}=\mathbb{R}^{n} \backslash \mathbb{Y}$. Then the sets

$$
\begin{array}{ll}
\mathbb{Y}_{\epsilon}=\{y \in \mathbb{Y} ; \operatorname{dist}(y, \mathbb{G})>\epsilon\}, & \text { for } 0<\epsilon \leqslant \kappa, \\
\mathbb{F}_{\epsilon}=\{y \in \mathbb{Y} ; \operatorname{dist}(y, \mathbb{G}) \geqslant \epsilon\}, & \text { for } 0<\epsilon \leqslant \kappa
\end{array}
$$

are connected.

Remark 8.1. This lemma fails if $\mathbb{Y}$ is not monotone.

It is easy to see that

$$
\partial \mathbb{Y}_{\epsilon} \subset \Gamma_{0}^{\epsilon} \cup \cdots \cup \Gamma_{\ell}^{\epsilon} \stackrel{\text { def }}{=} \Gamma^{\epsilon}=\left\{y \in \mathbb{Y} ; \operatorname{dist}\left(y, \mathbb{R}^{n} \backslash \mathbb{Y}\right)=\epsilon\right\} .
$$

In general, however, $\partial \mathbb{Y}_{\epsilon}$ may be a proper subset of $\Gamma_{0}^{\epsilon} \cup \cdots \cup \Gamma_{\ell}^{\epsilon}$, no matter how small $\epsilon$ is. We make this remark clear with an example of a snake shaped set as a boundary component of $\mathbb{Y}$; see Figure 2 , 
Remark 8.2. This example also illustrates that the boundary components of a monotone domain can be neither path connected nor locally connected. In our analysis we do not rule out such boundaries. It is worth noting that such rather bizarre boundaries are not present if the distance function

$$
\mathrm{d}_{\Omega}(y) \stackrel{\text { def }}{=} \operatorname{dist}(y, \mathbb{G}), \quad y \in \mathbb{Y},
$$

is strictly monotone near $\partial \mathbb{Y}$. The term strictly monotone refers to any function on $\mathbb{Y}$ which assumes neither local maximum nor local minimum. As regards the distance function, its local minimum is never attained in $\mathbb{Y}$. However, in Figure 2 the points in the dotted part of $\Gamma^{\epsilon}$ cannot move farther away from $\partial \mathbb{Y}$, meaning that these are local maxima for $d_{\mathbb{Y}}$.

It is not generally true that coordinates of a homeomorphism $h=\left(h^{1}, \ldots, h^{n}\right)$ : $\mathbb{X} \rightarrow \mathbb{Y}$ extend continuously to $\overline{\mathbb{X}}$. Nonetheless, some functions of $h$ do extend, such as $\operatorname{dist}(h(x), \partial \mathbb{Y})$. If a homeomorphism $h: \mathbb{X} \stackrel{\text { onto }}{\longrightarrow} \mathbb{Y}$ is fixed, it is not difficult to construct a continuous function $\Theta_{h}:[0, \infty) \rightarrow[0, \infty)$ increasing from 0 to $\infty$ such that

$$
\operatorname{dist}(h(x), \partial \mathbb{Y}) \leqslant \Theta_{h}\left(\operatorname{dist}\left(x, \mathbb{R}^{n} \backslash \mathbb{X}\right)\right), \quad \text { for all } x \in \mathbb{X} .
$$

Indeed the function $\Theta_{h}(t)=\sup \left\{d_{\mathbb{Y}} h(x), \operatorname{dist}\left(x, \mathbb{R}^{n} \backslash \mathbb{X}\right) \leqslant t\right\}$ works just as well. Without any additional regularity of the target domain one cannot say more than that. Let us take a moment for an example.

8.2. An example. We show that $\Theta_{h}$ can decrease to zero as slowly as we wish. Precisely, we have

Example 8.1. Given any continuous function $\Theta:[0, \infty) \rightarrow[0, \infty)$ increasing from zero to infinity, there exist $\mathbb{X}$ and $\mathbb{Y}$ and a homeomorphism $h: \mathbb{X} \rightarrow \mathbb{Y}$ of Sobolev class $\mathscr{W}^{1, n}(\mathbb{X}, \mathbb{Y})$ such that

$$
\lim _{t \rightarrow 0} \frac{\Theta_{h}(t)}{\Theta(t)}=\infty
$$

Proof. First we choose and fix a decreasing sequence of radii $1=r_{1}>r_{2}>\ldots$, small enough to satisfy

$$
\sum_{k=1}^{\infty} r_{k}<\infty \quad \text { and } \quad \Theta\left(r_{k}\right) \leqslant \frac{1}{2^{k}}, \quad \text { for } k=1,2, \ldots
$$

Then we set $R_{k+1}=k \Theta\left(r_{k}\right)$, with $R_{1}=1$. To define the domain $\mathbb{X}$ we consider an infinite tower of balls $\mathbb{D}_{k}=B\left(a_{k}, r_{k}\right)$, each on top of the previous one. Consecutive balls $\mathbb{D}_{k}$ and $\mathbb{D}_{k+1}$ are connected by a tiny passage so that $\mathbb{X}$ becomes a domain. These passages will play no role in computation since we can make them arbitrarily thin. In exactly the same way we construct an infinite tower $\mathbb{Y}$ with the balls $\mathbb{B}_{k} \stackrel{\text { def }}{=}$ $B\left(b_{k}, R_{k}\right)$ and the passages between them. Consider similarity transformations $h_{k}: \mathbb{D}_{k} \stackrel{\text { onto }}{\longrightarrow} \mathbb{B}_{k}, k=1,2, \ldots$. We assume that $h_{k+1}$ agrees with $h_{k}$ at the point where $\mathbb{D}_{k+1}$ meets $\mathbb{D}_{k}$. After making suitable extensions of $h_{k}$ to tiny passages, we obtain a mapping $h: \mathbb{X} \rightarrow \mathbb{Y}$ of finite conformal energy. Specifically, if one ignores 
the energy over the tiny passages, then the computation goes as follows:

$$
\begin{aligned}
\sum_{k=1}^{\infty} \int_{\mathbb{D}_{k}}\|D h\|^{n} & =n^{\frac{n}{2}} \sum_{k=1}^{\infty} \int_{\mathbb{D}_{k}} J(y, h) \mathrm{d} y=n^{\frac{n}{2}} \sum_{k=1}^{\infty} \operatorname{vol} \mathbb{B}_{k} \\
& \leqslant C_{n} \sum_{k=1}^{\infty} R_{k}^{n}=C_{n}\left(1+\sum_{k=1}^{\infty}\left[k \Theta\left(r_{k}\right)\right]^{n}\right) \\
& \leqslant C_{n}\left(1+\sum_{k=1}^{\infty} \frac{k^{n}}{2^{n k}}\right)<\infty .
\end{aligned}
$$

The remaining integrals over the passages can be made arbitrarily small. Now consider any $0<t<1$. Thus $t \in\left[r_{k+1}, r_{k}\right)$ for precisely one integer $k \geqslant 1$. Since the centers of the balls $\mathbb{D}_{k}$ are mapped into the centers of $\mathbb{B}_{k}$, we find that

$$
\Theta_{h}(t) \geqslant \Theta_{h}\left(r_{k+1}\right) \geqslant R_{k+1}=k \Theta\left(r_{k}\right) \geqslant k \Theta(t) .
$$

This yields

$$
\lim _{t \rightarrow 0} \frac{\Theta_{h}(t)}{\Theta(t)}=\infty
$$

8.3. Estimates near a monotone boundary, proof of Theorem 1.2. Here we give qualitatively sharp estimates near any monotone component of $\partial \mathbb{Y}$. Theorem 1.2 is then immediate from the estimates below.

Theorem 8.1. Let $\mathbb{X}$ have at least two boundary components or, otherwise, be incompressible. Suppose that $\mathbb{Y}$ is monotone near $\partial \mathbb{Y}_{i}$, for some $i=0,1, \ldots, \ell$. Then

$$
\operatorname{dist}^{n}\left(h(x), \mathbb{Y}_{i}\right) \leqslant \frac{C(\mathbb{X}, \mathbb{Y}) \int_{\mathbb{X}}|D h|^{n}}{\log \left(e+\frac{\operatorname{diam} \mathbb{X}}{\operatorname{dist}\left(x, \mathbb{X}_{i}\right)}\right)}
$$

for every $h \in \mathcal{E}(\mathbb{X}, \mathbb{Y})$.

Note that no regularity of $\partial \mathbb{X}_{i}$ is required.

Proof. We reexamine Cases 1, 2, 3, and 4 in Section 7.3, with a new choice of the potential function $U$.

Case 1. This case has already been established; see Remark 7.1

Case 2. Suppose $\mathbb{Y}$ is monotone near an inner component $\partial \mathbb{Y}_{i}$ of $\partial \mathbb{Y}$ for some $i \in\{1, \ldots, \ell\}$. We assume that the corresponding component of $\mathbb{R}^{n} \backslash \mathbb{X}$ satisfies

$$
\operatorname{diam} \mathbb{X}_{i}=2 d>0 .
$$

Choose and fix a positive small number $\kappa<\operatorname{dist}\left(\partial \mathbb{Y}_{\alpha}, \partial \mathbb{Y}_{i}\right)$. For all $\alpha \neq i$, the level sets

$$
\Gamma_{i}^{\epsilon}=\left\{y \in \mathbb{Y} ; \quad \operatorname{dist}\left(y, \partial \mathbb{Y}_{i}\right)=\epsilon\right\}, \quad 0 \leqslant \epsilon \leqslant \kappa,
$$

are all connected. We consider the potential function in $\mathbb{R}^{n}$,

$$
U(y)= \begin{cases}\operatorname{dist}\left(y, \mathbb{Y}_{i}\right) & \text { if } \operatorname{dist}\left(y, \mathbb{Y}_{i}\right) \leqslant \kappa, \\ \kappa & \text { otherwise. }\end{cases}
$$


Then we pull it back via the mapping $h: \mathbb{X} \stackrel{\text { onto }}{\longrightarrow} \mathbb{Y}$ by the rule

$$
u(x)= \begin{cases}0 & \text { for } x \in \mathbb{X}_{i}, \\ U(h(x)) & \text { if } x \in \mathbb{X} \text { and } U(h(x)) \leqslant \kappa, \\ \kappa & \text { otherwise. }\end{cases}
$$

This function is continuous in $\mathbb{R}^{n}$. As in Case 2 of Section 7.3 the function $u(x)$ is continuous and monotone on every ball $\mathbb{B}$ of diameter $2 d$. Each component of the level set $u(x) \equiv c, 0<c \leqslant \kappa$, is connected and separates $\partial \mathbb{X}_{i}$ from $\partial \mathbb{X}_{0}$. Thus it has diameter at least $d$. This time, as an advantage, we have a uniform bound of the gradient of $u$ on the entire domain $\mathbb{X}$ :

$$
|\nabla u(x)| \leqslant|D h(x)| \quad \text { in } \mathbb{X} .
$$

That is why we do not need to cut off $u$ near $\partial \mathbb{X}_{i}$. Moreover, $\nabla u \equiv 0$ outside $\mathbb{X}$. Using the inequality (26), we obtain

$$
|u(a)-u(b)|^{n} \leqslant \frac{C_{n} \int_{\mathbb{X}}|\nabla u|^{n}}{\log \left(e+\frac{d}{|a-b|}\right)} \leqslant \frac{C_{n} \int_{\mathbb{X}}|D h|^{n}}{\log \left(e+\frac{d}{|a-b|}\right)},
$$

provided $|a-b| \leqslant d$. Given any point $x \in \mathbb{X}$ with $\operatorname{dist}\left(x, \partial \mathbb{X}_{i}\right) \leqslant d$ and $\operatorname{dist}\left(h(x), \partial \mathbb{Y}_{i}\right)$ $\leqslant \kappa$, we apply (89) to $a=x$ and choose $b \in \partial \mathbb{X}_{i}$ such that $|a-b|=\operatorname{dist}\left(x, \partial \mathbb{X}_{i}\right)$ and

$$
\operatorname{dist}^{n}\left(h(x), \mathbb{Y}_{i}\right) \leqslant \frac{C_{n} \int_{\mathbb{X}}|D h|^{n}}{\log \left(e+\frac{d}{\operatorname{dist}\left(x, \mathbb{X}_{i}\right)}\right)} .
$$

Note that if $\operatorname{dist}\left(x, \mathbb{X}_{i}\right) \geqslant d$ and $\operatorname{dist}\left(h(x), \mathbb{Y}_{i}\right) \leqslant \kappa$, then the required estimate is trivial. Indeed, in such a case we have

$$
\begin{aligned}
\operatorname{dist}^{n}\left(h(x), \mathbb{Y}_{i}\right) & \leqslant[\operatorname{dist} \mathbb{Y}]^{n}=\frac{[\operatorname{diam} \mathbb{Y}]^{n}}{|\mathbb{Y}|} \int_{\mathbb{X}} J(x, h) \mathrm{d} x \\
& \leqslant \frac{[\operatorname{diam} \mathbb{Y}]^{n}}{|\mathbb{Y}|} \int_{\mathbb{X}}|D h|^{n} \\
& \leqslant \frac{[\operatorname{diam} \mathbb{Y}]^{n}}{|\mathbb{Y}|} \frac{2 \int_{\mathbb{X}}|D h|^{n}}{\log \left(e+\frac{d}{\operatorname{dist}\left(x, \mathbb{X}_{i}\right)}\right)} .
\end{aligned}
$$

We may summarize these two cases as

$$
\operatorname{dist}^{n}\left(h(x), \mathbb{Y}_{i}\right) \leqslant \frac{C(\mathbb{Y}) \int_{\mathbb{X}}|D h|^{n}}{\log \left(e+\frac{d}{\operatorname{dist}\left(x, \mathbb{X}_{i}\right)}\right)}
$$

whenever $\operatorname{dist}\left(h(x), \mathbb{Y}_{i}\right) \leqslant \kappa$. Finally, suppose that $\operatorname{dist}\left(h(x), \mathbb{Y}_{i}\right)>\kappa$. The level set $\Gamma=\left\{y \in \mathbb{Y} ; \operatorname{dist}\left(y, \mathbb{Y}_{i}\right)=\kappa\right\}$ separates $\mathbb{Y}_{i}$ from the point $h(x)$. Applying the inverse map $f=h^{-1}: \mathbb{Y} \rightarrow \mathbb{X}$ we see that $f(\Gamma)$ separates $\mathbb{X}_{i}$ from $x$. Take any point $x_{i} \in \partial \mathbb{X}_{i}$ which is the closest point to $x$. The straight line segment $\left[x_{i}, x\right]$ contains some $x^{\prime} \in f(\Gamma)$ for which

$$
\operatorname{dist}\left(x, \mathbb{X}_{i}\right)=\left|x-x_{i}\right| \geqslant\left|x^{\prime}-x_{i}\right| \geqslant \operatorname{dist}\left(x^{\prime}, \mathbb{X}_{i}\right) .
$$


Inequality (92) is applicable to $x^{\prime}$ because $\operatorname{dist}\left(h\left(x^{\prime}\right), \mathbb{Y}_{i}\right)=\kappa$. Hence,

$$
\begin{aligned}
\kappa^{n} & =\operatorname{dist}^{n}\left(h\left(x^{\prime}\right), \mathbb{Y}_{i}\right) \leqslant \frac{C(\mathbb{Y}) \int_{\mathbb{X}}|D h|^{n}}{\log \left(e+\frac{d}{\operatorname{dist}\left(x^{\prime}, \mathbb{X}_{i}\right)}\right)} \\
& \leqslant \frac{C(\mathbb{Y}) \int_{\mathbb{X}}|D h|^{n}}{\log \left(e+\frac{d}{\operatorname{dist}\left(x, \mathbb{X}_{i}\right)}\right)} .
\end{aligned}
$$

We then conclude with the desired estimate

$$
\begin{aligned}
\operatorname{dist}^{n}\left(h(x), \mathbb{Y}_{i}\right) & \leqslant[\operatorname{diam} \mathbb{Y}]^{n}=\frac{[\operatorname{diam} \mathbb{Y}]^{n}}{\kappa^{n}} \kappa^{n} \\
& \leqslant \frac{[\operatorname{diam} \mathbb{Y}]^{n}}{\kappa^{n}} \frac{C(\mathbb{Y}) \int_{\mathbb{X}}|D h|^{n}}{\log \left(e+\frac{d}{\operatorname{dist}\left(x, \mathbb{X}_{i}\right)}\right)}
\end{aligned}
$$

for every $x \in \mathbb{X}$. Estimates (92) and (94) may be summarized by saying that

$$
\operatorname{dist}^{n}\left(h(x), \mathbb{Y}_{i}\right) \leqslant \frac{C(\mathbb{Y}) \int_{\mathbb{X}}|D h|^{n}}{\log \left(e+\frac{\operatorname{diam} \mathbb{X}}{\operatorname{dist}\left(x, \mathbb{X}_{i}\right)}\right)}
$$

for every $x \in \mathbb{X}$.

Case 3. This case, being similar to Case 2, needs only clarification of the points of difference. Assume that either

$$
\operatorname{diam}\left(\mathbb{X}_{1} \cup \cdots \cup \mathbb{X}_{\ell}\right)=2 d>0, \quad \text { for } \ell \geqslant 1,
$$

or $\mathbb{X}$ is incompressible for $\ell=0$. We consider a condenser $(\Upsilon, \Omega)$ in the target space,

$$
\Omega=\mathbb{R}^{n} \backslash \mathbb{Y}_{0}=\mathbb{Y} \cup \mathbb{Y}_{1} \cup \cdots \cup \mathbb{Y}_{\ell}
$$

First, we construct a continuum $\Upsilon$. If $\ell \geqslant 1$, we take a continuum which contains $\mathbb{Y}_{1} \cup \cdots \cup \mathbb{Y}_{\ell}$. If $\ell=0$, we choose a continuum $\mathfrak{X} \subset \mathbb{X}$ of positive essential diameter and then take $\Upsilon \subset \mathbb{Y}$ to be the image of $\mathfrak{X}$ under a homeomorphism $g \in \mathcal{H}(\mathbb{X}, \mathbb{Y})$. In this case the parameter $d$ is determined by $2 d=\operatorname{diam}(\mathfrak{X}, \mathbb{X})$. The potential function $U$ is defined by the rule

$$
U(y)= \begin{cases}\operatorname{dist}\left(y, \mathbb{Y}_{0}\right) & \text { if } \operatorname{dist}\left(y, \mathbb{Y}_{0}\right) \leqslant \kappa, \\ \kappa & \text { otherwise. }\end{cases}
$$

Here $\kappa$ is small enough so that $\operatorname{dist}\left\{\Upsilon, \partial \mathbb{Y}_{0}\right\}>\kappa$. Since $\mathbb{Y}$ is monotone near $\partial \mathbb{Y}_{0}$ we may also assume, taking $\kappa$ even smaller, that (85) is satisfied for $i=0$. The remaining arguments are much the same as in Case 2.

Case 4 . We now come to the last case in which $\mathbb{R}^{n} \backslash \mathbb{X}$ has two components, the outer component $\mathbb{X}_{0}$ and a single point $\{a\}$. This case requires additional care because we want to achieve a linear dependence on the energy on the right hand side of formula (83). Fix a ball $B(a, 2 R) \subset \mathbb{X} \cup\{a\}$. We consider the same condenser $(\Upsilon, \Omega)$ as in Case 4 of Section 7.3.2, where $\Omega=\mathbb{Y}^{\prime}=\mathbb{Y} \cup\{b\}$ and $\Upsilon \subset \Omega$ contains a ball $\mathbb{B}(b, \delta)$ with $0<\delta<\operatorname{dist}\left(b, \mathbb{Y}_{0}\right)$. We define the potential function

$$
U(y)= \begin{cases}\operatorname{dist}\left(y, \mathbb{Y}_{0}\right) & \text { if } \operatorname{dist}\left(y, \mathbb{Y}_{0}\right) \leqslant \kappa, \\ \kappa & \text { otherwise. }\end{cases}
$$


Here $0<\kappa<\operatorname{dist}\left(b, \mathbb{Y}_{0}\right)-\delta$. We also assume that $\kappa$ is small enough so that the level sets $\Gamma_{t}=\{y \in \mathbb{Y} ; U(y)=t\}, 0<t \leqslant \kappa$, are connected. Certainly these level sets separate the ball $\mathbb{B}=\mathbb{B}(b, \delta)$ from $\mathbb{Y}_{0}$. Lemma 7.4 tells us that

$$
\operatorname{diam} h^{-1}\left(\Gamma_{t}\right) \geqslant 2 d>0, \quad 0<t \leqslant \kappa,
$$

where $d=\min \{R, r\}$ and

$$
\delta^{n}=\frac{C_{n} E}{\log \left(1+\frac{R}{r}\right)}
$$

see (70) and (71). The associated pullback of $U$ is defined by the rule

$$
u(x)= \begin{cases}0 & \text { if } x \notin \mathbb{X}^{\prime}, \\ U(h(x)) & \text { if } x \in \mathbb{X}^{\prime} \text { and } U(h(x)) \leqslant \kappa, \\ \kappa & \text { otherwise. }\end{cases}
$$

We note that $u$ is continuous and monotone on every ball of diameter $2 d$. Hence, we have the inequality

$$
|u(a)-u(b)|^{n} \leqslant \frac{C_{n} \int_{\mathbb{X}}|\nabla u|^{n}}{\log \left(e+\frac{d}{|a-b|}\right)} .
$$

Now, given $x \in \mathbb{X}$ such that $\operatorname{dist}\left(h(x), \mathbb{Y}_{0}\right) \leqslant \kappa$, the above inequality applies to the point $b \in \mathbb{X}_{0}$ which is the closest point (in $\mathbb{X}_{0}$ ) to $x$ :

$$
\operatorname{dist}^{n}\left(h(x), \mathbb{Y}_{0}\right) \leqslant \frac{C_{n} \int_{\mathbb{X}}|D h|^{n}}{\log \left(e+\frac{d}{\operatorname{dist}\left(x, \mathbb{X}_{0}\right)}\right)}
$$

What remains is to relax the assumption $\operatorname{dist}\left(h(x), \mathbb{Y}_{0}\right) \leqslant \kappa$. First, we use the same trick as in Case 2 to show that (102) is true for all $x \in \mathbb{X}$. However, the real problem we are facing here is to eliminate $d$ so that it depends not only on $\mathbb{X}$ but also on the energy of $h$. Precisely, we want to replace $d$ by diam $\mathbb{X}$ in order to conclude with the final estimate that

$$
\operatorname{dist}^{n}\left(h(x), \mathbb{Y}_{0}\right) \leqslant \frac{C_{n} \int_{\mathbb{X}}|D h|^{n}}{\log \left(e+\frac{\operatorname{diam} \mathbb{X}}{\operatorname{dist}\left(x, \mathbb{X}_{0}\right)}\right)}
$$

This is done in two steps.

Step 1. Suppose $\left(\frac{R}{r}\right)^{2} \leqslant \frac{\operatorname{diam} \mathbb{X}}{\operatorname{dist}\left(x, \mathbb{X}_{0}\right)}$. If $d=R$, then (103) is obvious. Thus we assume that $d=r$. In this case we have

$$
d \geqslant R \sqrt{\frac{\operatorname{dist}\left(x, \mathbb{X}_{0}\right)}{\operatorname{diam} \mathbb{X}}}
$$

so inequality (103) is a simple consequence of (102).

Step 2. If $\left(\frac{R}{r}\right)^{2} \geqslant \frac{\operatorname{diam} \mathbb{X}}{\operatorname{dist}\left(x, \mathbb{X}_{0}\right)} \geqslant 1$, then

$$
\begin{aligned}
\operatorname{dist}^{n}\left(h(x), \mathbb{Y}_{0}\right) & \leqslant \frac{[\operatorname{diam} \mathbb{Y}]^{n}}{\delta^{n}} \delta^{n}=\frac{[\operatorname{diam} \mathbb{Y}]^{n}}{\delta^{n}} \frac{C_{n} E}{\log \left(1+\frac{R}{r}\right)} \\
& \leqslant \frac{2[\operatorname{diam} \mathbb{Y}]^{n} C_{n} E}{\delta^{n} \log \left(e+\frac{\operatorname{diam} \mathbb{X}}{\operatorname{dist}\left(x, \mathbb{X}_{0}\right)}\right)}
\end{aligned}
$$

as desired. 


\section{REMARKS ON THE EXISTENCE OF A MINIMIZING MAP}

The aim of this section is first to briefly discuss the existence of mappings with smallest conformal energy. Second, we will examine the invertibility features of the extremal mappings.

9.1. Contenders. Although it appears at first sight that the direct method of the calculus of variations can be applied directly to our problems, it turns out that some details become more delicate. We emphasize that the mappings $h$ : $\mathbb{X} \stackrel{\text { onto }}{\longrightarrow} \mathbb{Y}$ are not prescribed on the boundary of $\mathbb{X}$ as in the classical theory of elasticity [5]. We allow "tangential slipping" while fixing the image of the boundary. As pointed out by J. M. Ball these conditions can be realized physically for an incompressible material confined in a box. We find ourselves forced to complete a family of homeomorphisms in weak topology of the Sobolev space $\mathscr{W}^{1, n}(\mathbb{X}, \mathbb{Y})$.

It should be noted that the notion of $c$-uniform convergence, when applied to a sequence $h_{j} \in \mathcal{E}(\mathbb{X}, \mathbb{Y})$, of bounded energy actually agrees with that of weak $\mathscr{W}^{1, n_{-}}$ convergence. This is due to the modulus of continuity estimates for monotone functions that have been discussed in Section 8. Indeed, let $\mathcal{E}^{M}(\mathbb{X}, \mathbb{Y}) \subset \mathcal{E}(\mathbb{X}, \mathbb{Y})$ denote the class of homeomorphism in $\mathcal{E}(\mathbb{X}, \mathbb{Y})$ whose conformal energy is controlled by a constant $M>0$. As a consequence of Lemma 3.1 we have

Lemma 9.1 (Equicontinuity). The family $\mathcal{E}^{M}(\mathbb{X}, \mathbb{Y})$ is equicontinuous on each compact subset $\mathbb{K} \subset \mathbb{X}$. In fact, we have a uniform estimate of the modulus of continuity,

$$
\left|h\left(x_{1}\right)-h\left(x_{2}\right)\right|^{n} \leqslant \frac{C_{n}(\mathbb{K}) \cdot M}{\log \left(e+\frac{\operatorname{diam} \mathbb{X}}{\left|x_{1}-x_{2}\right|}\right)} \quad \text { for all } \quad x_{1}, x_{2} \in \mathbb{K}
$$

By virtue of Ascoli's theorem we can now speak of $c$-uniform convergence of minimizing sequences. The direct method in the calculus of variations can now be put into effect so as to yield the existence of the minimizing map. The proof is routine. However, numerous important questions arise. The first question is at the heart of topology, as it concerns approximation of a mapping with homeomorphisms. We recall that limits of homeomorphisms are cellular mappings ([23] and [24]), meaning that the inverse image of a point is an intersection of a decreasing sequence of $n$-cells, the notion introduced by Morton Brown [13]. In fact, Armentrout showed [3] that cellular mappings of an $n$-manifold onto itself can be approximated with homeomorphisms; see also Siebenmann [58]. The second question combines topology and analysis. The task is to approximate a mapping $h \in \mathscr{W}^{1, n}(\mathbb{X}, \mathbb{Y})$ with homeomorphisms in the Sobolev class $\mathscr{W}^{1, n}(\mathbb{X}, \mathbb{Y})$ and control their conformal energy.

9.2. The image of $\mathbb{X}$ under the extremal map. The self-mappings $h_{k}(x)=$ $|x|^{k} x$ of the unit ball $\mathbb{B} \subset \mathbb{R}^{n}$ converge $c$-uniformly to zero, which lies inside the target domain. The reader may wish to notice that this sequence of homeomorphisms is bounded in the Sobolev class $\mathscr{W}^{1,1}(\mathbb{B}, \mathbb{B})$, but not in $\mathscr{W}^{1, p}(\mathbb{B}, \mathbb{B})$, with $p>1$. It is also possible that homeomorphisms $h_{k}: \mathbb{B} \stackrel{\text { onto }}{\longrightarrow} \mathbb{B}$ are bounded in $\mathscr{W}^{1, n}(\mathbb{B}, \mathbb{B})$ and still converge $c$-uniformly to a constant mapping $h: \mathbb{B} \rightarrow\left\{a_{\circ}\right\}$. In this case, however, the constant value $a_{\circ}$ must lie in $\partial \mathbb{B}$; see Example 2.1. These observations actually reflect a general fact that the limit of homeomorphisms of 
bounded $p$-harmonic energy, $p>1$, cannot take $\mathbb{X}$ into a compact subset in the target.

Proposition 9.1. Let $\mathbb{X}$ and $\mathbb{Y}$ be bounded domains in $\mathbb{R}^{n}$ and let $h_{k}: \mathbb{X} \stackrel{\text { onto }}{\longrightarrow} \mathbb{Y}$ be homeomorphisms converging c-uniformly to $h$. Suppose that $h(\mathbb{X})$ is relatively compact in $\mathbb{Y}$. Then

$$
\lim _{k \rightarrow \infty} \int_{\mathbb{X}}\left\|D h_{k}(x)\right\|^{p} d x=\infty, \quad \text { for every } p>1 .
$$

Proof. The key ingredient in our proof is the following capacity estimate which is known in Sobolev theory. Suppose $u \in \mathscr{W}_{0}^{1,1}(\mathbb{X})$ and $u(x) \geqslant \rho>0$ on a compact subset $\mathbb{K} \subset \mathbb{X}$. Then

$$
\int_{\mathbb{X}}|\nabla u(x)| \mathrm{d} x \geqslant \gamma_{n} \rho|\mathbb{K}|^{\frac{n-1}{n}},
$$

where $\gamma_{n}=\sqrt[n]{n^{n-1} \omega_{n-1}}$ is the isoperimetric constant.

Having this estimate we fix a number $0<\rho<\operatorname{dist}(h(\mathbb{X}), \partial \mathbb{Y})$ and apply (107) to

$$
u_{k}(x)=\min \left\{\rho, \operatorname{dist}\left(h_{k}(x), \partial \mathbb{Y}\right)\right\} ; \quad \text { thus }\left|\nabla u_{k}(x)\right| \leqslant\left\|D h_{k}(x)\right\| .
$$

Given any compact set $\mathbb{K} \subset \mathbb{X}$, for sufficiently large integers $k=k(\mathbb{K})$ we have $\operatorname{dist}\left(h_{k}(x), \partial \mathbb{Y}\right) \geqslant \rho$ for all $x \in \mathbb{K}$. This is obvious because $h_{k} \rightrightarrows h$, uniformly on $\mathbb{K}$. Thus $u_{k}(x) \equiv \rho$ on $\mathbb{K}$, whence we can write

$$
\int_{\mathbb{X} \backslash \mathbb{K}}\left\|D h_{k}(x)\right\| \mathrm{d} x \geqslant \int_{\mathbb{X} \backslash \mathbb{K}}\left|\nabla u_{k}(x)\right| \mathrm{d} x=\int_{\mathbb{X}}\left|\nabla u_{k}(x)\right| \mathrm{d} x \geqslant \gamma_{n} \rho|\mathbb{K}|^{\frac{n-1}{n}} .
$$

Hölder's inequality gives

$$
\int_{\mathbb{X}}\left\|D h_{k}(x)\right\|^{p} \mathrm{~d} x \geqslant \frac{\gamma_{n}^{p} \rho^{p}|\mathbb{K}|^{\frac{n p-p}{n}}}{|\mathbb{X} \backslash \mathbb{K}|^{p-1}} \text { for every compact } \mathbb{K} \subset \mathbb{X}
$$

The desired conclusion follows by letting $\mathbb{K}$ expand to the entire domain $\mathbb{X}$.

9.3. BV-right inverse of the limit, proof of Theorem 1.4. Throughout this section $\mathbb{X}$ and $\mathbb{Y}$ have at least two boundary components. We need to show that the right inverse of the limit map, denoted by $f$, has bounded variation. In fact, we shall see that the variation of the right inverse is controlled by the $\mathscr{L}^{1}$-norm of the cofactors of $D h$ :

$$
\begin{aligned}
\|f\|_{\mathscr{B} \mathscr{V}(\mathbb{Y})} & \stackrel{\text { def }}{=} \sup \left|\int_{\mathbb{Y}}\left[D^{T} \varphi(y)\right] f(y) \mathrm{d} y\right| \leqslant \int_{\mathbb{X}}\left\|D^{\sharp} h(x)\right\| \mathrm{d} x \\
& \leqslant|\mathbb{X}|^{\frac{1}{n}}\|D h\|_{\mathscr{L}^{n}(\mathbb{X})}^{n-1}<\infty .
\end{aligned}
$$

The supremum runs over all test mappings $\varphi \in \mathscr{C}_{\circ}^{\infty}\left(\mathbb{Y}, \mathbb{R}^{n}\right)$ with $\|\varphi\|_{\infty}=13$

Proof. Let $M$ denote the upper bound of the sequence $\left\|D h_{j}\right\| \mathscr{L}^{n}(\mathbb{X})$. We begin with the following identity for the inverse mappings $f_{j}: \mathbb{Y} \stackrel{\text { onto }}{\longrightarrow} \mathbb{X}$ :

$$
\int_{\mathbb{Y}}\left[D^{*} \varphi(y)\right] f_{j}(y) \mathrm{d} y=-\int_{\mathbb{X}}\left[D^{\sharp} h_{j}(x)\right] \varphi\left(h_{j}(x)\right) \mathrm{d} x,
$$

\footnotetext{
${ }^{3}$ For further reading about almost-everywhere injectivity in nonlinear elasticity, see [51, 60].
} 
where $D^{*} \varphi$ stands for the transpose of $D \varphi$ and $D^{\sharp} h_{j}$ for the cofactor matrix of $D h_{j}$.

Proof of (110). We consider functions $H_{k} \in \mathscr{W}_{\mathrm{loc}}^{1, n}\left(\Omega, \mathbb{R}^{n}\right)$,

$$
H_{k}(x)=\left(h_{j}^{1}, \ldots, h_{j}^{k-1}, \omega, h_{j}^{k+1}, \ldots, h_{j}^{n}\right), \quad \text { where } \omega(x)=\sum_{i=1}^{n} x_{i} \varphi^{i}(h(x)) .
$$

The point is that the $k^{t h}$ coordinate of $H_{k}$ has compact support. Therefore,

$$
\int_{\mathbb{X}} J\left(x, H_{k}\right) \mathrm{d} x=0 .
$$

We then look at the vector field $V(x)=\left(J\left(x, H_{1}\right), \ldots, J\left(x, H_{n}\right)\right)$. An elementary computation shows that

$$
V(x)=\left[D^{\sharp} h_{j}(x)\right] \varphi+J\left(x, h_{j}\right)\left[D^{*} \varphi\right] x,
$$

where the vector field $\varphi$ and the matrix field $\left[D^{*} \varphi\right]$ are evaluated at the point $y=h_{j}(x)$. Since $\int_{\Omega} V(x) \mathrm{d} x=0 \in \mathbb{R}^{n}$, it holds that

$$
\int_{\mathbb{X}} J\left(x, h_{j}\right)\left[D^{*} \varphi\right] x \mathrm{~d} x=-\int_{\mathbb{X}}\left[D^{\sharp} h_{j}(x)\right] \varphi \mathrm{d} x .
$$

We now make a change of variables $y=h_{j}(x)$ on the left hand side. This step is legitimate because $h_{j}$ is a homeomorphism of the Sobolev class $\mathscr{W}_{\text {loc }}^{1, n}\left(\Omega, \mathbb{R}^{n}\right)$; see [55. Corollary 1, p. 182]. Accordingly,

$$
\int_{\mathbb{Y}}\left[\left(D^{*} \varphi\right)(y)\right] f_{j}(y) \mathrm{d} y=-\int_{\mathbb{X}}\left[D^{\sharp} h_{j}(x)\right] \varphi\left(h_{j}(x)\right) \mathrm{d} x .
$$

This completes the proof of the identity (110).

Returning to the proof of (109) we note that the identity (110) yields a bound independent of $j$,

$$
\begin{aligned}
\left|\int_{\mathbb{Y}}\left[D^{*} \varphi(y)\right] f_{j}(y) \mathrm{d} y\right| & \leqslant\|\varphi\|_{\infty}|\mathbb{X}|^{\frac{1}{n}}\left\|D h_{j}\right\|_{\mathscr{L}^{n}(\mathbb{X})}^{n-1} \\
& \leqslant\|\varphi\|_{\infty}|\mathbb{X}|^{\frac{1}{n}} M^{n-1}
\end{aligned}
$$

where $M$ stands for the upper bound of the $\mathscr{L}^{n}$-norms of $\left\{D h_{j}\right\}$. Next, taking supremum over all test mappings gives a bound for the $B V$-norm of $f_{j}$,

$$
\left\|f_{j}\right\|_{\mathscr{B} \mathscr{V}(\mathbb{Y})} \leqslant|\mathbb{X}|^{\frac{1}{n}} M^{n-1} .
$$

It is a well-known fact concerning $B V$-functions that for every Lipschitz subdomain $\mathbb{U} \subset \mathbb{Y}$ the inclusion $\mathscr{B} \mathscr{V}(\mathbb{U}) \subset \mathscr{L}^{p}(\mathbb{U})$ is continuous if $1 \leqslant p \leqslant \frac{n}{n-1}$. This inclusion is actually compact for $p<\frac{n}{n-1}$; see [1, Corollary 3.49, p. 152]. Thus we may pass to the limit with a subsequence of $\left\{f_{j}\right\}$ to obtain a mapping $f \in \mathscr{B} \mathscr{V}(\mathbb{U})$. We also obtain a BV-estimate independent of $\mathbb{U}$ :

$$
\|f\|_{\mathscr{B} \mathscr{V}(\mathbb{U})} \leqslant|\mathbb{X}|^{\frac{1}{n}} M^{n-1} .
$$

This is still not good enough for (109); we have to replace $M$ by the norm $\|D h\|_{\mathscr{L}^{n}(\mathbb{X})}$ of the limit map. However, with the aid of the uniform bounds at (115), we will 
be able to pass to the limit in the identity (110). Passing to the limit on the left hand side poses no difficulty. To deal with the right hand side we make use of weak continuity of the cofactors. We have $D^{\sharp} h_{j} \rightarrow D^{\sharp} h$ weakly in $\mathscr{L}^{\frac{n}{n-1}}\left(\mathbb{X}, \mathbb{R}^{n \times n}\right) \underline{4}$ On the other hand, $\varphi \circ h_{j} \rightarrow \varphi \circ h$ in $\mathscr{L}^{p}\left(\mathbb{X}, \mathbb{R}^{n}\right)$, for every $1 \leqslant p<\infty$. Now, the limit of (113) takes the form

$$
\int_{\mathbb{Y}}\left[D^{T} \varphi(y)\right] f(y) \mathrm{d} y=-\int_{\mathbb{X}}\left[D^{\sharp} h(x)\right] \varphi(h(x)) \mathrm{d} x
$$

for all $\varphi \in \mathscr{C}_{\circ}^{\infty}\left(\mathbb{Y}, \mathbb{R}^{n}\right)$. This yields (109) 5 Since $f_{j} \rightarrow f$ in $\mathscr{L}^{p}\left(\mathbb{Y}, \mathbb{R}^{n}\right), 1 \leqslant p<$ $\frac{n}{n-1}$, we know that $\left\{f_{j}\right\}$ contains a subsequence (again denoted by $f_{j}$ ) converging to $f$ almost everywhere. Up to now, $f$ is defined only almost everywhere; exactly at the points where $\lim _{j \rightarrow \infty} f_{j}(y)$ exists. To define $f$ for every $y \in \mathbb{Y}$ we look at the sequence $x_{j}=f_{j}(y) \in \mathbb{X}$. The key is the following remark:

$(\ddagger)$ Given any compact $\mathbb{K} \subset \mathbb{Y}$, the sets $f_{1}(\mathbb{K}), f_{2}(\mathbb{K}), \ldots$ stay away from the boundary of $\mathbb{X}$.

Precisely, by Theorem 1.1 we have

$$
\operatorname{dist}(\mathbb{K}, \partial \mathbb{Y})=\operatorname{dist}\left(h_{j}\left(f_{j}(\mathbb{K})\right), \partial \mathbb{Y}\right) \preccurlyeq M \log ^{-\frac{1}{n}}\left(e+\frac{\operatorname{diam} \mathbb{X}}{\operatorname{dist}\left(f_{j}(\mathbb{K}), \partial \mathbb{X}\right)}\right) .
$$

Now we apply this inequality to $\mathbb{K}=\{y\}$ to see that the points $x_{j}=f_{j}(y)$ stay away from $\partial \mathbb{X}$. We choose, arbitrarily, an accumulation point of $\left\{x_{j}\right\}$ to be the value of $f(y)$, say $f(y)=x=\lim _{\nu \rightarrow \infty} x_{i_{\nu}}$. Obviously $f$ is measurable. The identity (19) is straightforward. Indeed,

$$
h(f(y))=\lim _{\nu \rightarrow \infty} h_{j_{\nu}}(x)=\lim _{\nu \rightarrow \infty} h_{j_{\nu}}\left(x_{j_{\nu}}\right)=y
$$

because $h_{j} \rightrightarrows h, c$-uniformly. In a similar fashion we argue for the inclusion $\mathbb{Y} \subset$ $h(\mathbb{X})$. Given any $y \in \mathbb{Y}$, consider any cluster point of $\left\{f_{j}(y)\right\}$, say $x=\lim _{\nu \rightarrow \infty} f_{j_{\nu}}(y) \in$ $\mathbb{X}$. Obviously, $y=h(x) \in h(\mathbb{X})$. The proof of Theorem 1.4 is complete.

Remark 9.1. We observe that $h$, being a $c$-uniform limit of homeomorphisms, is monotone in the sense of C. B. Morrey [48]. For an excellent old survey about monotone mappings we refer to L. F. McAuley [46]. In view of Remark 3.1] we also see that $h$ is differentiable almost everywhere.

\section{COMPRESSION-EXPANSION PROCESS}

Throughout this section $\Omega$ will be a bounded domain in $\mathbb{R}^{n}$ of finite connectivity. We shall distinguish two open regions in $\mathbb{R}^{n}, \Omega^{+}=\Omega$ and $\Omega^{-}=\mathbb{R}^{n} \backslash \bar{\Omega}$, and denote their common boundary by $\Gamma=\partial \Omega=\overline{\Omega^{+}} \cap \overline{\Omega^{-}}$. It is convenient to view $\Omega$ as a container filled with gas or fluid. Accordingly, the application of an external pressure (from $\Omega^{-}$) compresses $\Omega$ into its subset, while the internal pressure (from $\Omega^{+}$) expands $\Omega$ into a superset. Having in mind the principle of nonpenetration of matter, we shall assume that the compression and expansion are injective.

\footnotetext{
${ }^{4}$ Weak continuity of the Jacobians and lower order minors go back at least to R. Caccioppoli 14] and C. B. Morrey [4]. Nowadays, this subject has been developed profitably in nonlinear elasticity [5], quasiconformal geometry [55, 30, 20], and calculus of variations [50].

${ }^{5}$ The reader may wish to observe at this point that the stronger estimate, stated in (108), actually holds.
} 
Definition 10.1. A compression-expansion process of $\Omega$ is a homotopy (continuous function) $F:(-T, T) \times \Omega \rightarrow \mathbb{R}^{n}$ such that

- $F_{\mathrm{o}}=\mathrm{id}: \Omega \rightarrow \Omega$,

- $F_{t}: \Omega \rightarrow \mathbb{R}^{n}$ is injective for $-T<t<T$.

Here we adopted the usual notation $F=F_{t}(y)$, for $-T<t<T$ and $y \in \Omega$.

A useful special case arises when one is able to compress or expand $\Omega$ into its level sets. We shall work with the following oriented distance function:

$$
\mathrm{d}(y)= \begin{cases}\operatorname{dist}(y, \Gamma) & \text { if } y \in \Omega^{+}, \\ 0 & \text { if } y \in \Gamma, \\ -\operatorname{dist}(y, \Gamma) & \text { if } y \in \Omega^{-} .\end{cases}
$$

A compression-expansion process is said to be equidistant if for every $-T<t<T$

$$
F_{t}(\Omega)=\Omega_{t} \stackrel{\text { def }}{=}\left\{y \in \mathbb{R}^{n} ; \quad d(y)>t\right\} .
$$

We aim to prove the following theorem.

Theorem 10.1. Every bounded graph domain $\Omega \subset \mathbb{R}^{n}$ is uniformly compressible and expansible near its boundary. Precisely, there exists a continuous map $F$ : $(-T, T) \times \mathbb{R}^{n} \rightarrow \mathbb{R}^{n}$ such that

- $F_{\circ}=\mathrm{id}: \mathbb{R}^{n} \rightarrow \mathbb{R}^{n}$, for all $t \in(-T, T)$,

- $F_{t}: \mathbb{R}^{n} \stackrel{\text { onto }}{\longrightarrow} \mathbb{R}^{n}$ is a homeomorphism, $-T<t<T$,

- $F_{t}(\Omega)=\Omega_{t}$, for all $t \in(-T, T)$.

Definition 10.2 (Graph domain). An open bounded connected set $\Omega \subset \mathbb{R}^{n}$ is a graph domain if near every point $a \in \partial \Omega$ there exists a rectangular coordinate system in which $\partial \Omega$ is a graph of a function and $\Omega$ lies on one side of the graph.

Corollary 10.1. Every graph domain $\Omega \subset \mathbb{R}^{n}$ is monotone.

Proof of of Corollary 10.1. First we note that $F_{t}(\Gamma)=\left\{y \in \mathbb{R}^{n} ; \mathrm{d}(y)=t\right\}$. Let $\Gamma_{0}, \Gamma_{1}, \ldots, \Gamma_{\ell}$ denote the components of $\Gamma$, and $\Gamma_{0}^{t}, \Gamma_{1}^{t}, \ldots, \Gamma_{\ell}^{t}$ denote the components of the level set $\left\{y \in \mathbb{R}^{n} ; \mathrm{d}(y)=t\right\}$. Since $F_{t}: \mathbb{R}^{n} \stackrel{\text { onto }}{\longrightarrow} \mathbb{R}^{n}$ is a homeomorphism, there is a one-to-one correspondence between $F_{t}\left(\Gamma_{0}\right), \ldots, F_{t}\left(\Gamma_{\ell}\right)$ and $\Gamma_{0}^{t}, \Gamma_{1}^{t}, \ldots, \Gamma_{\ell}^{t}$. On the other hand $F_{0}=i d$, so for sufficiently small $t$ we obtain $F_{0}^{t}=F_{t}\left(\Gamma_{0}\right), \ldots, F_{\ell}^{t}=F_{t}\left(\Gamma_{\ell}\right)$. The latter sets, being continuous images of connected sets, are also connected.

The proof of Theorem 10.1 is preceded by a number of geometric considerations.

Remark 10.1. The vertical line test asserts that to every point $a \in \Gamma$ there correspond a unit vector $v$ (the direction of the vertical line) and a neighborhood $\mathbb{O}$ with the following properties:

1. $\Gamma$ splits $\mathbb{O}$ into two components, $\mathbb{O}^{+}=\mathbb{O} \cap \Omega^{+}$(the upper component) and $\mathbb{O}^{-}=\mathbb{O} \cap \Omega^{-}$(the lower component), so that $\mathbb{O} \backslash \Gamma=\mathbb{O}^{+} \cup \mathbb{O}^{-}$and $\mathbb{O} \cap \Gamma=\overline{\mathbb{O}^{+}} \cap \overline{\mathbb{O}^{-}}$.

2. There is $\delta>0$ such that $t v+\overline{\mathbb{O}^{+}} \subset \Omega^{+}$if $0<t<2 \delta$. Thus slightly lifting up the closer of the upper component will place it in the open region $\Omega^{+}$. Similarly, $t v+\overline{\mathbb{O}^{-}} \subset \Omega^{-}$if $-2 \delta<t<0$. 
Lemma 10.1. Given a point $a \in \Gamma$ there is a ball $\mathbb{B}=B(a, r)$ with small radius so that for every $y \in \mathbb{B}$ the function

$$
t \rightarrow \mathrm{d}(y+t v), \quad-r<t<r,
$$

is strictly increasing.

Proof of Lemma 10.1. We choose and fix $0<r<\delta$ such that $B(a, 4 r) \subset \mathbb{O}$. This yields that $y+t v \in B(a, 2 r) \subset \mathbb{O}$. Let us examine two distinct values of the variable $t,-r<t_{1}<t_{2}<r$. We are going to show that $\mathrm{d}\left(y+t_{1} v\right)<\mathrm{d}\left(y+t_{2} v\right)$.

Case 1. Suppose $\mathrm{d}\left(y+t_{1} v\right) \stackrel{\text { def }}{=} \rho>0$. Thus $y+t_{1} v \in \Omega^{+}$and $\rho=\operatorname{dist}\left(y+t_{1} v, \Gamma\right)$. Since $a \in \Gamma$, we find that $\rho \leqslant\left|y+t_{1} v-a\right| \leqslant|y-a|+\left|t_{1}\right|<r+r=2 r$. The closed ball $\mathbb{D}=\left\{z ;\left|z-\left(y+t_{1} v\right)\right| \leqslant \rho\right\}$ lies in the closure of $\Omega^{+}$, by the definition of $\rho$. For each $z \in \mathbb{D}$, we have

$$
|z-a| \leqslant\left|z-\left(y+t_{1} v\right)\right|+|y-a|+\left|t_{1}\right|<\rho+r+r<4 r .
$$

This yields

$$
\mathbb{D} \subset B(a, 4 r) \cap \overline{\Omega^{+}} \subset \mathbb{O} \cap \overline{\Omega^{+}} \subset \overline{\mathbb{O}^{+}} .
$$

We now lift up $\mathbb{D}$ by the vector $\left(t_{2}-t_{1}\right) v$, where $0<t_{2}-t_{1}<2 r<2 \delta$. In view of declaration 2 in Remark 10.1 we see that the closed ball $\left(t_{2}-t_{1}\right) v+\mathbb{D}$ lies in $\Omega^{+}$. It is an open region. Therefore, the distance of the center $\left(t_{2}-t_{1}\right) v+\left(y+t_{1} v\right)=y+t_{2} v$ of this ball to the boundary $\Gamma=\partial \Omega^{+}$is strictly greater than its radius $\rho$. This summarizes to

$$
\mathrm{d}\left(y+t_{2} v\right)>\rho=\operatorname{dist}\left(y+t_{1} v\right),
$$

as desired.

The same proof works if $\rho=0$. However, in this case $\mathbb{D}$ degenerates into a point. We are left with the case:

Case 2. Suppose $\mathrm{d}\left(y+t_{1} v\right)<0$. There is nothing to prove if $\mathrm{d}\left(y+t_{2} v\right) \geqslant 0$, so we may assume that $\mathrm{d}\left(y+t_{2} v\right) \stackrel{\text { def }}{=}-\rho<0$. This means that $y+t_{2} v \in \Omega^{-}$and that $\rho=\operatorname{dist}\left(y+t_{2} v, \Gamma\right)$. The rest of the arguments are the same as in Case 1 , so we only state the conclusion

$$
\operatorname{dist}\left(y+t_{1} v, \Gamma\right)>\rho=\operatorname{dist}\left(y+t_{2} v, \Gamma\right) .
$$

This simply means that

$$
\mathrm{d}\left(y+t_{1} v, \Gamma\right)<\mathrm{d}\left(y+t_{2} v, \Gamma\right),
$$

completing the proof of Lemma 10.1

Since $\Gamma$ is compact we may cover it by a finite number of balls, denoted by $\mathbb{B}_{1}=B\left(a_{1}, r_{1}\right), \mathbb{B}_{2}=B\left(a_{2}, r_{2}\right), \ldots, \mathbb{B}_{m}=B\left(a_{m}, r_{m}\right)$, each of which satisfies the assertion of Lemma 10.1. Precisely, to every $\mathbb{B}_{i}$ there corresponds a unit vector $v_{i}$ such that the function

$$
t \rightarrow \mathrm{d}\left(y+t v_{i}\right), \quad-r<t<r, \quad r=\min \left\{r_{1}, \ldots, r_{m}\right\},
$$

is increasing whenever $y \in \mathbb{B}_{i}$. It turns out that the function $d$ remains strictly increasing in some directions different from $v_{1}, \ldots, v_{m}$. 
Lemma 10.2. Let $y \in \mathbb{B}_{k_{1}} \cap \mathbb{B}_{k_{2}} \cap \cdots \cap \mathbb{B}_{k_{\ell}}$ and

$$
v=\lambda_{1} v_{k_{1}}+\cdots+\lambda_{\ell} v_{k_{\ell}}, \quad \lambda_{1}, \ldots, \lambda_{\ell}>0 .
$$

Then the function

$$
t \rightarrow \mathrm{d}(y+t v)
$$

is strictly increasing near zero.

Proof. Since $y$ can be any point in $\mathbb{B}_{k_{1}} \cap \mathbb{B}_{k_{2}} \cap \cdots \cap \mathbb{B}_{k_{\ell}}$, we need only prove that $\mathrm{d}(y+\epsilon v)>\mathrm{d}(y)$ for sufficiently small $\epsilon>0$. To this end, we take $\epsilon$ small enough so that $\epsilon \lambda_{1}<r, \ldots, \epsilon \lambda_{\ell}<r$ and so that all points

$$
\begin{aligned}
y_{\circ} & =y \\
y_{1} & =y+\epsilon \lambda_{1} v_{k_{1}}, \\
y_{2} & =y+\epsilon \lambda_{1} v_{k_{1}}+\epsilon \lambda_{2} v_{k_{2}} \\
& \vdots \\
y_{\ell} & =y+\epsilon \lambda_{1} v_{k_{1}}+\ldots+\epsilon \lambda_{\ell} v_{k_{\ell}}=y+\epsilon v
\end{aligned}
$$

belong to $\mathbb{B}_{k_{1}} \cap \cdots \cap \mathbb{B}_{k_{\ell}}$. We repeatedly use Lemma 10.1 to obtain a chain of inequalities $\mathrm{d}(y+\epsilon v)=\mathrm{d}\left(y_{\ell}\right)=\mathrm{d}\left(y_{\ell-1}+\epsilon \lambda_{\ell} v_{k_{\ell}}\right)>\mathrm{d}\left(y_{\ell-1}\right)>\cdots>\mathrm{d}\left(y_{1}\right)=$ $\mathrm{d}\left(y_{\circ}+\epsilon \lambda_{1} v_{k_{1}}\right)>\mathrm{d}\left(y_{\circ}\right)=\mathrm{d}(y)$, as desired. The lemma is proved.

Next we fix nonnegative smooth functions $\varphi_{k} \in \mathscr{C}_{\circ}^{\infty}\left(\mathbb{B}_{k}\right), k=1, \ldots, \ell$, such that $\varphi_{1}(y)+\cdots+\varphi_{k}(y)>0$ for $y \in \Gamma$, and we define

$$
V(y)=\sum_{k=1}^{m} \varphi_{k}(y) v_{k} \in \mathscr{C}_{\circ}^{\infty}\left(\mathbb{R}^{n}, \mathbb{R}^{n}\right) .
$$

Lemma 10.3. The vector field $V$ does not vanish on $\Gamma$.

Proof. For a given point $y \in \Gamma$ we express $V(y)$ as

$$
V(y)=\varphi_{k_{1}}(y) v_{k_{1}}+\cdots+\varphi_{k_{\ell}}(y) v_{k_{\ell}}
$$

where we selected all the coefficients in (121) which are strictly positive at $y$. This, in particular, means that $y \in \mathbb{B}_{k_{1}} \cap \cdots \cap \mathbb{B}_{k_{\ell}}$. Then, by Lemma 10.2, for sufficiently small $\epsilon>0$ we have $\mathrm{d}(y+\epsilon v)>\mathrm{d}(y)$. This yields $v \neq 0$ and the lemma follows.

We shall work in a neighborhood of $\Gamma$ where $V \neq 0$. Denote it by

$$
\mathbb{U}=\left\{y \in \mathbb{R}^{n} ; \quad V(y) \neq 0\right\} .
$$

Through every point $y_{\circ} \in \mathbb{R}^{n}$ there passes exactly one integral curve $\gamma=\{y(t) ;-\infty$ $<t<\infty\}$, where $y=y(t)$ solves the equation

$$
\left\{\begin{array}{l}
\dot{y}(t)=V(y(t)), \quad-\infty<t<\infty \\
y\left(t_{\circ}\right)=y_{\circ}
\end{array}\right.
$$

Note that $y(t)$ is constant if $y_{\circ} \in \mathbb{R}^{n} \backslash \mathbb{U}$. In this case $\gamma$ reduces to one point $\left\{y_{\circ}\right\}$.

Lemma 10.4. Let $y=y(t)$ be an integral curve. Then the function $\rho(t)=\mathrm{d}(y(t))$ is nondecreasing. 
Proof. Choose and fix a point $a \in \mathbb{R}$. First we observe that

$$
\rho(t)-\rho(a) \geqslant o(t-a) \quad \text { as } t \searrow a .
$$

Indeed, using Taylor's expansion of $y(t)$ near $a$, in view of Lipschitz continuity of the distance function, we obtain

$$
\begin{aligned}
\rho(t)-\rho(a) & =\mathrm{d}(y(t))-\mathrm{d}(y(a)) \\
& =\mathrm{d}(y(a)+\dot{y}(a)(t-a)+o(t-a))-\mathrm{d}(y(a)) \\
& =\mathrm{d}(y(a)+(t-a) \dot{y}(a))-\mathrm{d}(y(a))+o(t-a) .
\end{aligned}
$$

Clearly (123) holds if $\dot{y}(a)=0$. If $\dot{y}(a) \neq 0$, then we express it as

$$
\dot{y}(a)=\sum_{k=1}^{m} \varphi_{k}(y(a)) v_{k}=\lambda_{1} v_{k_{1}}+\cdots+\lambda_{\ell} v_{k_{\ell}} .
$$

Here again we selected all positive coefficients $\lambda_{1}=\varphi_{k_{1}}(y(a)), \ldots, \lambda_{\ell}=\varphi_{k_{\ell}}(y(a))$. In particular, $y(a) \in \mathbb{B}_{k_{1}} \cap \cdots \cap \mathbb{B}_{k_{\ell}}$. By Lemma 10.2 we conclude with the inequality

$$
\mathrm{d}(y(a)+(t-a) \dot{y}(a))>\mathrm{d}(y(a))
$$

for $t$ sufficiently close to $a$. Inequality (123) follows. On the other hand, we note that $\rho$ is Lipschitz continuous and differentiable almost everywhere. Moreover,

$$
|\dot{\rho}(t)| \leqslant|\dot{y}(t)| \leqslant \sum_{k=1}^{m} \varphi_{k}(y(t)) \leqslant\left\|\varphi_{1}+\cdots+\varphi_{m}\right\|_{\infty} \stackrel{\text { def }}{=} L .
$$

We aim to show that $\dot{\rho}(t) \geqslant 0$ for almost every $t \in \mathbb{R}$. Assume, on the contrary, that there is an $\epsilon>0$ for which the set $J=\{t \in \mathbb{R} ; \quad \dot{\rho}(t) \leqslant-2 \epsilon\}$ has positive measure. Let $a \in J$ be a density point of $J$; that is,

$$
\lim _{t \searrow a} \frac{|T \cap(a, t)|}{t-a}=1 .
$$

Now the required contradiction follows from the inequality

$$
\begin{aligned}
\rho(t)-\rho(a) & =\int_{a}^{t} \dot{\rho}(\tau) \mathrm{d} \tau=\int_{J \cap(a, t)} \dot{\rho}(\tau) \mathrm{d} \tau+\int_{(a, t) \backslash J} \dot{\rho}(\tau) \mathrm{d} \tau \\
& \leqslant-2 \epsilon|J \cap(a, t)|+|(a, t) \backslash J| \cdot L \leqslant-(t-a) \epsilon \neq o(t-a) .
\end{aligned}
$$

Next we are able to improve Lemma 10.4 .

Lemma 10.5. For each integral curve $y=y(t)$ the function $\rho(t)=\mathrm{d}(y(t))$ is strictly increasing as long as $y(t) \in \mathbb{U}$.

Proof. Suppose that $\rho(t)$ is constant in an open (nonempty) interval $I$ such that $y(t) \in \mathbb{U}$ for all $t \in I$. Thus, in particular, $\dot{\rho}(t)=0$ for almost every $t \in I$. To reach a contradiction we consider the equation

$$
\dot{y}(t)=V(y(t))=\sum_{k=1}^{m} \varphi_{k}(y(t)) v_{k} \neq 0 \quad \text { for all } t \in I .
$$

Here the direction field does not vanish because $y(t) \in \mathbb{U}$. We shall conveniently narrow $I$ to reach a nonempty subinterval $I_{+} \subset I$ such that

$$
\dot{y}(t)=\varphi_{k_{1}}(y(t)) v_{k_{1}}+\cdots+\varphi_{k_{\ell}}(y(t)) v_{k_{\ell}}, \quad \text { for } t \in I_{+},
$$

where all the coefficients are strictly positive on $I_{+}$. This can be achieved in the following steps. Let us begin by discarding all the first terms in (128) which vanish 
identically on $I$, say $\varphi_{1} \equiv \cdots \equiv \varphi_{k_{1}-1} \equiv 0$. Since $\dot{y}(t) \neq 0$, some terms are still left. The first remaining coefficient $\varphi_{k_{1}}=\varphi_{k_{1}}(y(t))$ is positive on some open (nonempty) subinterval $I_{1} \subset I$. We then discard all the terms after $\varphi_{k_{1}}$, say $\varphi_{k_{1}+1}=\cdots=\varphi_{k_{2}-1}=0$, which vanish identically on $I_{1}$. Thus $\varphi_{k_{2}}=\varphi_{k_{2}}(y(t))$ is positive on some open (nonempty) subinterval $I_{2} \subset I_{1}$. Continuing in this fashion we end up with the interval $I_{+}=I_{1} \cap I_{2} \cap \cdots \cap I_{\ell}$ in which the formula (129) holds with positive coefficients.

Now, since $\rho(t)$ is constant on $I_{+}$, we shall reach a contradiction if we fix a point $a \in I_{+}$and show that $\mathrm{d}(y(t))>\mathrm{d}(y(a))$ for all $t>a$ sufficiently close to $a$. The entire curve $y=y(t)$ with $t \in I_{+}$lies in the affine space $y(a)+\mathcal{V}$ where $\mathcal{V}=\operatorname{span}\left\{v_{k_{1}}, \ldots, v_{k_{\ell}}\right\}$. Moreover, $y(a) \in \mathbb{B}_{k_{1}} \cap \cdots \cap \mathbb{B}_{k_{\ell}}$. Select a basis $\left\{v_{1}^{\prime}, \ldots, v_{s}^{\prime}\right\} \subset\left\{v_{k_{1}}, \ldots, v_{k_{\ell}}\right\}$ for the space $\mathcal{V}$. Taylor's expansion formula for $y(t)$ at the point $a$ takes the form

$$
y(t)=y(a)+(t-a) v .
$$

Here $v=v(t)=\dot{y}(a)+o(1)$ as $t \searrow a$. It is also important that $v$ lies in $\mathcal{V}$. Since $v_{1}^{\prime}, \ldots, v_{s}^{\prime}$ are linearly independent, we find that

$$
v(t)-\dot{y}(a)=\epsilon_{1}(t) v_{1}^{\prime}+\cdots+\epsilon_{s}(t) v_{s}^{\prime},
$$

where all the coefficients $\epsilon_{1}, \ldots, \epsilon_{s}$ approach zero as $t \searrow a$; some may be negative. We find that

$$
\begin{aligned}
v(t) & =\varphi_{k_{1}}(y(a)) v_{k_{1}}+\cdots+\varphi_{k_{\ell}}(y(a)) v_{k_{\ell}}+\epsilon_{1}(t) v_{1}^{\prime}+\cdots+\epsilon_{s}(t) v_{s}^{\prime} \\
& =\lambda_{1}(t) v_{k_{1}}+\cdots+\lambda_{k_{\ell}}(t) v_{k_{\ell}}, \quad t>a,
\end{aligned}
$$

with all coefficients $\lambda_{1}(t), \ldots, \lambda_{k_{\ell}}(t)$ strictly positive if $t$ is sufficiently close to $a$. Finally, by Lemma 10.2, we conclude that

$$
\rho(t)=\mathrm{d}(y(t))=\mathrm{d}(y(a)+(t-a) v)>\mathrm{d}(y(a))=\rho(a) .
$$

Thus $\rho$ cannot be constant on $I_{+}$. This contradiction proves Lemma 10.5

Through any point $z \in \mathbb{R}^{n}$ there passes exactly one integral curve. We denote and parametrize it as

$$
\gamma_{z}=\{y=y(t), \dot{y}(t)=V(y(t)), \text { for }-\infty<t<\infty, \text { and } y(0)=z\} .
$$

From now on we restrict the parameter $t$ to the interval $-1 \leqslant t \leqslant 1$. In other words, the integral curves are cut at the points $y(1)$ and $y(-1)$. Let us examine two functions defined for $z \in \mathbb{R}^{n}$ by the rules

$$
\begin{aligned}
& \mathrm{d}^{+}(z)=\mathrm{d}(y(1))-\mathrm{d}(z) \geqslant 0, \\
& \mathrm{~d}^{-}(z)=\mathrm{d}(y(-1))-\mathrm{d}(z) \leqslant 0 .
\end{aligned}
$$

The above inequalities hold because $\mathrm{d}(y(t))$ is nondecreasing. These functions are continuous. To this effect we notice that both $y(1)$ and $y(-1)$ depend smoothly on $z$. In fact, the entire solution $y=y(t),-\infty<t<\infty$, depends smoothly on the initial data $y(0)=z$. Now it is obvious that $\mathrm{d}^{+}(z)$ and $\mathrm{d}^{-}(z)$ are compactly supported. They are Lipschitz continuous and vanish outside $\mathbb{U}$. More importantly, $\mathrm{d}^{+}(z)>0$ and $\mathrm{d}^{-}(z)<0$ for $z \in \mathbb{U}$, because $\mathrm{d}(y(t))$ is strictly increasing as long as $y(t) \in \mathbb{U}$. Since $\Gamma=\partial \Omega \subset \mathbb{U}$ is compact, there exists a small positive number $T>0$ such that

$$
\mathrm{d}^{+}(z)>2 T \quad \text { and } \quad \mathrm{d}^{-}(z)<-2 T
$$


whenever $z \in \mathbb{U}_{T} \stackrel{\text { def }}{=}\{z ; \operatorname{dist}(z, \Gamma)<T\}$. We now define a function $\omega:[-T, T] \times$ $\mathbb{U}_{T} \rightarrow \mathbb{U}_{T}$. Precisely, for $t \in[-T, T]$ and $z \in \mathbb{U}_{T}$ we set $\omega=\omega(t, z)$ to be the unique point on the integral curve $\gamma_{z}$ such that $\mathrm{d}(\omega)=t$. To see the existence of such a point $\omega \in \mathbb{U}_{T}$ (uniqueness is obvious), we consider the parametrization of $\gamma_{z}=\{y(s) ; \quad \dot{y}(s)=V(y(s))\},-\infty<s<\infty$, such that $z=y(0)$. Then we consider the function $\rho(s)=\mathrm{d}(y(s))$, with $-1 \leqslant s \leqslant 1$, to find that

$$
\rho(1)=\mathrm{d}^{+}(z)+\mathrm{d}(z) \geqslant 2 T-T=T
$$

and

$$
\rho(-1)=\mathrm{d}^{-}(z)+\mathrm{d}(z) \leqslant-2 T+T=-T .
$$

Since $s \rightarrow \rho(s)$ is continuous and strictly increasing, there exists exactly one parameter $s=s(t) \in[-1,1]$ such that $\rho(s)=t \in[-T, T]$. We define

$$
\omega(t, z)=y(s) \in \mathbb{U}_{T}
$$

for $-T<t<T$ and $z \in \mathbb{U}_{T}$. Let us prove that

Lemma 10.6. The function $\omega=\omega(t, z)$ is continuous on $(-T, T) \times \mathbb{U}_{T}$.

Proof. If this were not so, there would exist parameters $t_{j} \rightarrow t \in(-T, T)$ and points $z_{j} \rightarrow z \in \mathbb{U}_{T}$, where $-T<t_{j}<T$ and $z_{j} \in \mathbb{U}_{T}, j=1,2, \ldots$, such that

$$
\omega_{j} \stackrel{\text { def }}{=} \omega\left(t_{j}, z_{j}\right) \rightarrow \omega_{\circ} \neq \omega \stackrel{\text { def }}{=} \omega(t, z) \text {. }
$$

Here, both points $\omega_{j}$ and $z_{j}$ lie in the same integral curve, say $\gamma_{j}$. Also $\omega$ and $z$ lie in their own integral curve, say $\gamma$. In describing the passage from the curves $\gamma_{j}$ to the limit curves $\gamma$, we note that the points $z_{j}=y_{j}(0)$ converge to $z=y(0)$, so $y_{j}(s)-y(s) \rightrightarrows 0$ uniformly for $-1 \leqslant s \leqslant 1$. This is immediate from the general fact that the solutions $y_{j}=y_{j}(t)$ depend continuously (actually smoothly) on the initial data. In particular, $y_{j}\left(s_{j}\right)-y\left(s_{j}\right) \rightarrow 0$ whenever $s_{j} \in[-1,1]$. We determine $s_{j} \in[-1,1]$ uniquely by the conditions $y_{j}\left(s_{j}\right)=\omega_{j} \in \gamma_{j}$ and $\mathrm{d}\left(\omega_{j}\right)=t_{j}$. Passing to a subsequence we may assume that $s_{j} \rightarrow s \in[-1,1]$, so as to obtain

$$
y(s)=\lim y\left(s_{j}\right)=\lim y_{j}\left(s_{j}\right)=\lim \omega_{j}=\omega_{\mathrm{o}} \in \gamma .
$$

Thus $t=\lim t_{j}=\lim \mathrm{d}\left(\omega_{j}\right)=\mathrm{d}\left(\omega_{\circ}\right)=\mathrm{d}\left(\lim \omega_{j}\right)$. On the other hand, by the definition of $\omega=\omega(t, z) \in \gamma$ we have $\mathrm{d}(\omega)=t$. In conclusion, we obtained two points $\omega_{0}, \omega \in \gamma$ such that $\mathrm{d}\left(\omega_{0}\right)=\mathrm{d}(\omega)=t$. Hence $\omega_{\circ}=\omega$; this contradiction completes the proof of Lemma 10.6

Corollary 10.2. The boundary of a graph domain is a neighborhood retract.

Proof. The retraction $\gamma: \mathbb{U}_{T} \rightarrow \Gamma$ of a neighborhood $\mathbb{U}_{T}$ of $\Gamma$ is given by the rule $z \rightarrow \omega(0, z)$ for $z \in \mathbb{U}_{T}$.

Proof of Theorem 10.1. We shall now complete the proof of Theorem 10.1, To this end we construct the compression-expansion map $F:(-T, T) \times \mathbb{R}^{n} \rightarrow \mathbb{R}^{n}$ by using $\omega:(-T, T) \times \mathbb{U}_{T} \rightarrow \mathbb{U}_{T}:$

$$
\begin{cases}F(\tau, z)=z & \text { for } z \in \mathbb{R}^{n} \backslash \mathbb{U}_{T},-T<\tau<T, \\ F(\tau, z)=\omega(t, \tau) & \text { for } z \in \mathbb{U}_{T},-T \leqslant \tau \leqslant T,\end{cases}
$$

where $t=\tau+\mathrm{d}(z)-\frac{\tau}{T}|\mathrm{~d}(z)| \in(-T, T)$. This latter inclusion can easily be seen since the function $d \rightarrow \tau+d-\frac{\tau}{T}|d|$ is strictly increasing for $-T<d<T$. Moreover, 
it assumes its minimum and maximum value at the endpoints of the interval, that is, for $d= \pm T$. Let us check that $F(t, z)$ is well defined and continuous. We need only verify compatibility of the above two formulas $F(\tau, z)$ at the points $z \in \partial \mathbb{U}_{T}$. This means that $\mathrm{d}(z)= \pm T$, depending on whether $z \in \Omega^{+}$or $z \in \Omega^{-}$. In either case $t=\mathrm{d}(z)$ so $\omega(t, z)=z$, by the definition of $\omega(t, z)$. Hence the verification of compatibility is complete.

We now check that for $\tau$ fixed the mapping $z \rightarrow F(\tau, z)$ is injective. Suppose that $F\left(\tau, z_{1}\right)=F\left(\tau, z_{2}\right)$. The case $F\left(\tau, z_{1}\right)=F\left(\tau, z_{2}\right) \in \mathbb{R}^{n} \backslash \mathbb{U}_{T}$ is trivial because $z_{1}=F\left(\tau, z_{1}\right)=F\left(\tau, z_{2}\right)=z_{2}$. Thus we assume that $F\left(\tau, z_{1}\right)=F\left(\tau, z_{2}\right) \in \mathbb{U}_{T}$. This yields that $\omega\left(t_{1}, z_{1}\right)=\omega\left(t_{2}, z_{2}\right)$, where

$$
t_{1}=\tau+\mathrm{d}\left(z_{1}\right)-\frac{\tau}{|T|}\left|\mathrm{d}\left(z_{1}\right)\right| \quad \text { and } \quad t_{2}=\tau+\mathrm{d}\left(z_{2}\right)-\frac{\tau}{|T|}\left|\mathrm{d}\left(z_{2}\right)\right| .
$$

Moreover, both points $z_{1}$ and $z_{2}$ belong to the same integral curve. By the definition of $\omega(t, z)$ we have $t_{1}=\mathrm{d}\left(\omega\left(t_{1}, z_{1}\right)\right)=\mathrm{d}\left(\omega\left(t_{2}, z_{2}\right)\right)=t_{2}$. Hence

$$
\tau+\mathrm{d}\left(z_{1}\right)-\frac{\tau}{T}\left|d\left(z_{1}\right)\right|=\tau+\mathrm{d}\left(z_{2}\right)-\frac{\tau}{T}\left|d\left(z_{2}\right)\right| .
$$

As we have already noticed the function $d \rightarrow \tau+d-\frac{\tau}{T}|d|$ is strictly increasing. Hence $\mathrm{d}\left(z_{1}\right)=\mathrm{d}\left(z_{2}\right)$. But this equation holds only when $z_{1}=z_{2}$, because $z_{1}$ and $z_{2}$ lie in the same integral curve. In conclusion, the mappings $F_{\tau}(z)=F(\tau, z)$ are injective in $\mathbb{R}^{n}$ for all $-T<\tau<T$.

On the other hand, it is a general fact that a continuous injection $F_{\tau}: \mathbb{R}^{n} \stackrel{\text { into }}{\longrightarrow}$ $\mathbb{R}^{n}$ which is the identity map outside a compact subset must be a homeomorphism of $\mathbb{R}^{n}$ onto $\mathbb{R}^{n}$. Thus, for each parameter $\tau \in[-T, T]$ the mapping $F_{\tau}: \mathbb{R}^{n} \rightarrow \mathbb{R}^{n}$ is bijective. This map is the identity outside $\mathbb{U}_{T}$,

$$
F_{\tau}: \mathbb{U}_{T} \stackrel{\text { onto }}{\longrightarrow} \mathbb{U}_{T} \quad \text { and } \quad F_{\tau}=\mathrm{id}: \partial \mathbb{U}_{T} \rightarrow \partial \mathbb{U}_{T} .
$$

For $\tau=0$, we have $F_{\circ}=\mathrm{id}: \mathbb{R}^{n} \rightarrow \mathbb{R}^{n}$. Indeed, given any point $z \in \mathbb{U}_{T}$ we define $F_{\circ}(z)=\omega(t, z)$ where $t=0+\mathrm{d}(z)-\frac{0}{T}|\mathrm{~d}(z)|=\mathrm{d}(z)$, so $\omega(t, z)=z$.

More generally, the image of $\Omega$ under the map $F_{\tau},-T<\tau<T$, is the level set

$$
\Omega_{\tau}=\left\{z \in \mathbb{R}^{n} ; \quad \mathrm{d}(z)>\tau\right\}, \quad-T<\tau<T .
$$

Indeed, given any point $z \in \partial \Omega=\Gamma$, we have $\mathrm{d}(z)=0$, so $F(\tau, z)=\omega=\omega(t, z)$, where $t=\tau+\mathrm{d}(z)-\frac{\tau}{T}|\mathrm{~d}(z)|=\tau$. This means that $\mathrm{d}(\omega)=\tau$, which means $\omega \in \partial \Omega_{\tau}$, as required.

\section{REFERENCES}

[1] Ambrosio, L., Fusco, N., and Pallara, D. Functions of bounded variation and free discontinuity problems. Oxford Mathematical Monographs, 2000. MR.1857292(2003a:49002)

[2] Antman, S. S. Fundamental mathematical problems in the theory of nonlinear elasticity. Univ. Maryland, College Park, Md., 1975, 35-54. MR0468503 (57:8335)

[3] Armentrout, S. Concerning cellular decompositions of 3-manifolds that yield 3-manifolds. Trans. Amer. Math. Soc. 133 (1968), 307-332. MR0230296 (37:5859)

[4] Astala, K., Iwaniec, T., Martin, G. J., and Onninen, J. Extremal mappings of finite distortion. Proc. London Math. Soc. (3) 91 (2005), no. 3, 655-702. MR2180459 (2006h:30016)

[5] Ball, J. M. Convexity conditions and existence theorems in nonlinear elasticity. Arch. Rational Mech. Anal. 63 (1978), 337-403. MR0475169(57:14788) 
[6] Ball, J. M. Global invertibility of Sobolev functions and the interpenetration of matter. Proc. Roy. Soc. Edinburgh Sect. A 88, no. 3-4, (1981), 315-328. MR616782 (83f:73017)

[7] Bojarski, B., and Iwaniec, T. Analytical foundations of the theory of quasiconformal mappings in $R^{n}$. Ann. Acad. Sci. Fenn. Ser. A I Math. 8 (1983), no. 2, 257-324. MR731786|(85h:30023)

[8] Borsuk, K. Über Schnitte der n-dimensionalen Euklidischen Räume. Math. Ann. 106 (1932), no. 1, 239-248. MR 1512757

[9] Borsuk, K. Drei Sätze über die n-dimensionale Euklidische Sphäre. Fund. Math. 20 (1933), 177-190.

[10] Borsuk, K. Set theoretical approach to the disconnection theory of the Euclidean space. Fund. Math. 37 (1950), 217-241. MR0042699 (13:150d)

[11] Borsuk, K. Theory of retracts. Monografie Matematyczne, Tom 44 Państwowe Wydawnictwo Naukowe, Warsaw, 1967. MR0216473 (35:7306)

[12] Brouwer, L. E. J. Beweis des Jordanschen Satzes für den n-dimensionalen Raum. Math. Ann. 71 (1911), no. 3, 314-319. MR.1511659

[13] Brown, M. The monotone union of open $n$-cells is an open $n$-cell. Proc. Amer. Math. Soc. 12 (1961), 812-814. MR0126835 (23:A4129)

[14] Caccioppoli, R. Funzioni pseudo-analitiche e rappresentazioni pseudo-conformi delle superficie riemanniane. (Italian) Ricerche Mat. 2 (1953), 104-127. MR0062845 (16:27c)

[15] Carathéodory, C. Über die gegenseitige Beziehung der Ränder bei der konformen Abbildung des Inneren einer Jordanschen Kurve auf einen Kreis. Math. Ann. 73 (1913), 305-320. MR1511735

[16] Ciarlet, P. G. Mathematical elasticity. Vol. I. Three-dimensional elasticity. Studies in Mathematics and its Applications, 20, North-Holland Publishing Co., Amsterdam, 1988. MR936420 (89e:73001)

[17] Csörnyei, M., Hencl, S, and Malý, J. Homeomorphisms in the Sobolev space $W^{1, n-1}$. J. Reine Angew. Math. 644 (2010), 221-235. MR2671780

[18] Duren, P. Harmonic mappings between planar domains, Cambridge Tracts in Mathematics, 156, Cambridge University Press, Cambridge, 2004. MR.2048384 (2005d:31001)

[19] Gehring, F. W., and Lehto, O. On the total differentiability of functions of a complex variable. Ann. Acad. Sci. Fenn. Ser. A I No. 272 (1959). MR0124487 (23:A1799)

[20] Giannetti, F., Iwaniec, T., Onninen, J., and Verde, A. Estimates of Jacobians by subdeterminants. J. Geom. Anal. 12 (2002), no. 2, 223-254. MR1888516 (2002k:42042)

[21] Gol'dstein V. M., and Vodop'yanov S. K. Quasiconformal mappings and spaces of functions with generalized first derivatives. Sibirsk. Mat. Z. 17 (1976), 515-531. MR.0414869 (54:2961)

[22] Hajłasz, P., and Malý, J. Approximation in Sobolev spaces of nonlinear expressions involving the gradient. Ark. Mat. 40 (2002), no. 2, 245-274. MR1948065 (2003m:46052)

[23] Haver, W. Cellular mappings on manifolds. Ph.D. Dissertation, State University of New York at Binghamton, ProQuest LLC, Ann Arbor, MI, 1971, 60pp. MR2620456

[24] Haver, W. A characterization theorem for cellular maps. Bull. Amer. Math. Soc. 76 (1970), 1277-1280. MR0267538 (42:2440)

[25] Heinonen, J., Kilpeläinen, T., and Martio, O. Nonlinear potential theory of degenerate elliptic equations. Oxford Mathematical Monographs, 1993. MR.1207810 (94e:31003)

[26] Heinonen, J., and Koskela, P. Sobolev mappings with integrable dilatations. Arch. Rational Mech. Anal. 125 (1993), no. 1, 81-97. MR.1241287 (94i:30020)

[27] Hencl, S., and Koskela, P. Regularity of the inverse of a planar Sobolev homeomorphism. Arch. Ration. Mech. Anal. 180 (2006), no. 1, 75-95. MR2211707(2007e:30020)

[28] Iwaniec, T., Koskela, P., and Onninen, J. Mappings of finite distortion: Monotonicity and continuity. Invent. Math. 144 (2001), no. 3, 507-531. MR.1833892 (2002c:30029)

[29] Iwaniec, T., and Martin, G. J. Geometric Function Theory and Non-linear Analysis. Oxford Mathematical Monographs, 2001. MR,1859913 (2003c:30001)

[30] Iwaniec, T., and Onninen, J. $\mathcal{H}^{1}$-estimates of Jacobians by subdeterminants. Math. Ann. 324 (2002), no. 2, 341-358. MR1933861 (2003i:42027)

[31] Iwaniec, T., and Onninen, J. n-Harmonic mappings between annuli. Mem. Amer. Math. Soc., to appear.

[32] Iwaniec, T., and Onninen, J. Hyperelastic deformations of smallest total energy. Arch. Rational Mech. Anal. 194 (2009), no. 3, 927-986. MR2563629(2010j:49005) 
[33] Iwaniec, T., and Onninen, J. Deformations of finite conformal energy: Existence, and removability of singularities. Proc. Lond. Math. Soc. (3), 100 (2010), no. 1, 1-23. MR2578466 (2011a:30063)

[34] Iwaniec, T. and Šverák, V. On mappings with integrable dilatation. Proc. Amer. Math. Soc. 118 (1993), no. 1, 181-188. MR.1160301 (93k:30023)

[35] Kauhanen, J., Koskela, P., and Malý, J. Mappings of finite distortion: Discreteness and openness. Arch. Ration. Mech. Anal. 160 (2001), no. 2, 135-151. MR1864838(2002m:30023)

[36] Kovalev, L. V., and Onninen J. Boundary values of mappings of finite distortion. Rep. Univ. Jyväskylä Dep. Math. Stat. 92 (2003), 175-182. MR2061003 (2005d:30032)

[37] Kuratowski, K. Topology. Vol. I. Academic Press, New York-London; Państwowe Wydawnictwo Naukowe, Polish Scientific Publishers, Warsaw, 1966. MR0217751 (36:840)

[38] Kuratowski, K. Topology. Vol. II. Academic Press, New York-London; Państwowe Wydawnictwo Naukowe, Polish Scientific Publishers, Warsaw, 1968. MR0259835 (41:4467)

[39] Kuusalo, T. Quasiconformal mappings without boundary extensions. Ann. Acad. Sci. Fenn. Ser. A I Math. 10 (1985), 331-338. MR802494(87a:30035)

[40] Lafont, J. F. Strong Jordan separation and applications to rigidity. J. London Math. Soc. (2) 73 (2006), no. 3, 681-700. MR2241974 (2007h:53057)

[41] Lafont, J. F. A note on strong Jordan separation. Publ. Mat. 53 (2009), no. 2, 515-525. MR2543863 (2010h:57036)

[42] Lebesgue, H. Sur le problème de Dirichlet. Rend. Circ. Palermo 27 (1907), 371-402.

[43] Malý, J., and Martio, O. Lusin's condition ( $N$ ) and mappings of the class $W^{1, n}$. J. Reine Angew. Math. 458 (1995), 19-36. MR.1310951 (95m:26024)

[44] Manfredi, J. J. Weakly monotone functions. J. Geom. Anal. 4 (1994), no. 3, 393-402. MR1294334 (95m:30032)

[45] Manfredi, J. J., and Villamor, E. Mappings with integrable dilatation in higher dimensions. Bull. Amer. Math. Soc. (N.S.) 32 (1995), no. 2, 235-240. MR1313107 (95m:30033)

[46] McAuley, L. F. The Proceedings of the First Conference on Monotone Mappings and Open Mappings. Edited by Louis F. McAuley. State University of New York at Binghamton, 1971. MR0287518 (44:4722)

[47] Menchoff, D. Sur les différentielles totales des fonctions univalentes. Math. Ann. 105 (1931), 75-85. MR.1512705

[48] Morrey, C.B. The Topology of (Path) Surfaces. Amer. J. Math. 57 (1935), no. 1, 17-50. MR 1507053

[49] Morrey, C.B. Quasi-convexity and the lower semicontinuity of multiple integrals. Pacific J. Math. 2 (1952), 25-53. MR0054865(14:992a)

[50] Müller, S. Weak continuity of determinants and nonlinear elasticity. C. R. Acad. Sci. Paris Sér. I Math. 307 (1988), no. 9, 501-506. MR964116 (90b:49023)

[51] Müller, S., Spector, S. J., and Tang, Q. Invertibility and a topological property of Sobolev maps. SIAM J. Math. Anal. 27 (1996), no. 4, 959-976. MR.1393418 (97e:46041)

[52] Näkki, R. Boundary behavior of quasiconformal mappings in n-space. Ann. Acad. Sci. Fenn. Ser. A I Math. 484 (1970), 1-50. MR0285707 (44:2925)

[53] Onninen, J. Regularity of the inverse of spatial mappings with finite distortion, Calc. Var. Partial Differential Equations 26 (2006), no. 3, 331-341. MR:2232209 (2007b:30023)

[54] Osgood, W. F. and Taylor, E. H. Conformal transformations on the boundaries of their regions of definition. Trans. Amer. Math. Soc. 14 (1913), 277-298. MR1500947

[55] Reshetnyak, Yu. G. Space mappings with bounded distortion. Translations of Mathematical Monographs, 73. American Mathematical Society, Providence, RI, 1989. MR994644 (90d:30067)

[56] Rickman, S. Quasiregular mappings. Springer-Verlag, Berlin, 1993. MR.1238941 (95g:30026)

[57] Sard, A. The measure of the critical values of differentiable maps. Bull. Am. Math. Soc. 48 (1942), 883-890. MR0007523 (4:153c)

[58] Siebenmann, L. C. Approximating cellular maps by homeomorphisms. Topology 11 (1972), 271-294. MR0295365 (45:4431)

[59] Šverák, V. Regularity properties of deformations with finite energy. Arch. Rational Mech. Anal. 100 (1988), no. 2, 105-127. MR.913960 (89g:73013)

[60] Tang, Q. Almost-everywhere injectivity in nonlinear elasticity. Proc. Roy. Soc. Edinburgh Sect. A 109 (1988), no. 1-2, 79-95. MR952330 (89g:73014) 
[61] Wilder, R. L. Topology of Manifolds. American Mathematical Society Colloquium Publications, vol. 32. American Mathematical Society, New York, N. Y., 1949. MR.0029491(10:614c)

[62] Vuorinen, M. Conformal geometry and quasiregular mappings. Lecture Notes in Mathematics, 1319. Springer-Verlag, Berlin, 1988. MR950174 (89k:30021)

[63] Väisälä, J. Lectures on n-dimensional quasiconformal mappings. Lecture Notes in Mathematics, Vol. 229, Springer-Verlag, Berlin-New York, 1971. MR.0454009 (56:12260)

Department of Mathematics, Syracuse University, Syracuse, New York 13244

E-mail address: tiwaniec@syr.edu

Department of Mathematics, Syracuse University, Syracuse, New York 13244

E-mail address: jkonnine@syr.edu 